\title{
WestVirginiaUniversity
}

THE RESEARCH REPOSITORY @ WVU

Graduate Theses, Dissertations, and Problem Reports

2006

\section{Shape based automated mapping of histological mouse brain sections}

Manasa Danda

West Virginia University

Follow this and additional works at: https://researchrepository.wvu.edu/etd

\section{Recommended Citation}

Danda, Manasa, "Shape based automated mapping of histological mouse brain sections" (2006).

Graduate Theses, Dissertations, and Problem Reports. 4223.

https://researchrepository.wvu.edu/etd/4223

This Thesis is protected by copyright and/or related rights. It has been brought to you by the The Research Repository @ WVU with permission from the rights-holder(s). You are free to use this Thesis in any way that is permitted by the copyright and related rights legislation that applies to your use. For other uses you must obtain permission from the rights-holder(s) directly, unless additional rights are indicated by a Creative Commons license in the record and/ or on the work itself. This Thesis has been accepted for inclusion in WVU Graduate Theses, Dissertations, and Problem Reports collection by an authorized administrator of The Research Repository @ WVU. For more information, please contact researchrepository@mail.wvu.edu. 


\title{
Manasa Danda
}

\author{
Thesis submitted to the \\ College of Engineering and Mineral Resources at \\ West Virginia University \\ in partial fulfillment of the requirements \\ for the Degree of
}

MASTER OF SCIENCE

IN

ELECTRICAL ENGINEERING

Dr. Donald A. Adjeroh, Chair

Dr. Aric Agmon

Dr. Mark A. Jerabek

Dr. Xin Li

Lane Department of Computer Science and Electrical Engineering

Morgantown, West Virginia

2006

Keywords:

Brain Atlas, Feature Extraction, Shape Matching, Fourier Descriptors, Image Mapping, Shape Classification

Copyright 2006 Manasa Danda 


\section{ABSTRACT \\ SHAPE BASED AUTOMATIC MATCHING OF HISTOLOGICAL SECTIONS}

\section{Manasa Danda}

In the development of an automatic image mapping system for mouse brain images, we focus on obtaining the best match in the two dimensional atlas images for the query image of a mouse brain section. The matching issue has to deal with the tissue distortions and tears, which are routinely encountered and possible scale, rotation and shear changes. With the goal to achieve high performance for image matching, a dual-stage approach is proposed which is based on radial distributions and region-based template matching.

Our main challenge is identifying shape features and corresponding distance metrics that produce effective characterization of 2D sections for similarity comparison. The mapping algorithm analyses the shape characteristics of target models and perform a similarity measurement against database templates. In this technique, the first stage makes use of the contour of the brain section and intrinsic local geometric features to estimate the approximate location of the given section along the brain axis. Here, we considered three approaches: morphometric-based features, Fourier based descriptors and radial distributions. In the second stage, a content-based approach is adopted in which we use texture and localization features to make a final decision about the mapping. More specifically, we studied morphometric-based methods and the use of Gabor filters for this fine-grained analysis. The statistical distribution of these features constitutes the essential concept for achieving a match between reference and subject images.

With the efficient integration of the stages, our proposed approach for histological brain mapping gives the benefits of both speed and accuracy. Comparative results with other proposed methods are studied. 


\section{Acknowledgements}

I would like to express my deep respect and profound thanks to Dr. Donald Adjeroh. Any words cannot truly express my gratitude for the opportunities, encouragement and knowledge that you have bestowed upon me and for the skills that I have gathered through my interaction with you. I have learnt a lot from you both professionally and personally.

Dr. Aric Agmon, it was great to work with you on the Neuroinformatics part of this work. I wish to express my gratitude to Professor Joseph Morton for his help and support.

I thank the members of the committee Dr.Xin Li and Dr.Mark Jerabek for their guidance and valuable inputs. I also thank all the faculty members of the WVU CSEE department and WVU Tech CSEE department.

My colleague Umashankar Kandaswamy deserves big thanks as well. Umasankar, thanks for being a friend and for all the interesting insights during the course of this work. I thank my friends Zola and Bhyrav for their help and support.

I am fortunate for the life and guidance that my parents have given me. Their support was invaluable when I formed my desire to pursue this line of study. None of this would have been possible but for their sacrifices. I also express my deepest gratitude to my sister Swetha Danda for her undying support and understanding during the past few years.

\section{Manasa Danda}

July 2006 


\section{TABLE OF CONTENTS}

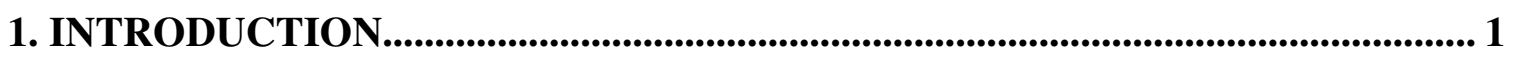

1.1 MOTIVATION

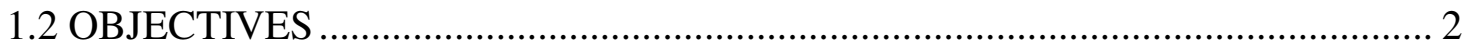

1.3 AUTOMATED MOUSE BRAIN MATCHING SYSTEM …………………........ 3

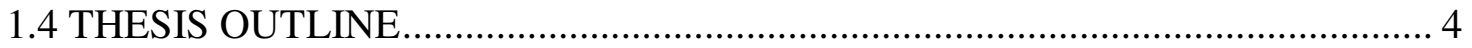

2. BACKGROUND AND RELATED WORK ..................................................................... 5

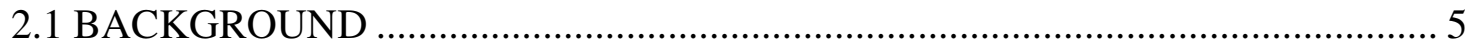

2.1.2 Mouse Brain Atlas................................................................................ 5

2.1.3 Current Research ................................................................................. 6

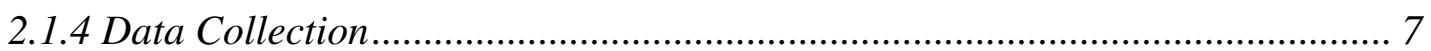

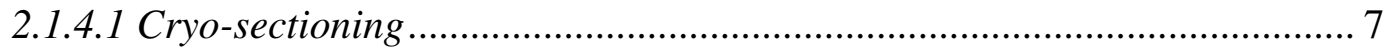

2.1.4.2 Image Acquisition .............................................................................. 8

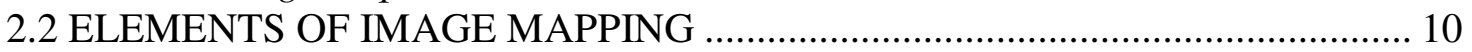

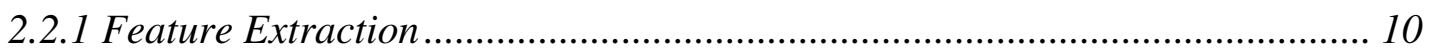

2.2.2 Similarity Measure ................................................................................. 12

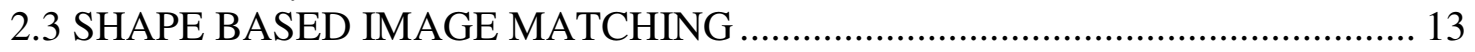

2.3.1 Shape Representation.............................................................................. 13

2.3.1.1 Contour-Based Shape Representation Techniques .................................. 14

2.3.1.2 Region-Based Shape Representation Techniques ..................................... 16

2.3.2 Shape Similarity Measures ............................................................................ 17

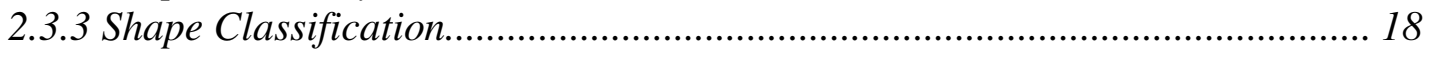

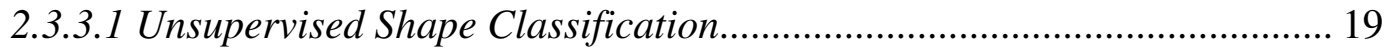

2.3.3.2 Supervised Shape Classification ............................................................. 19

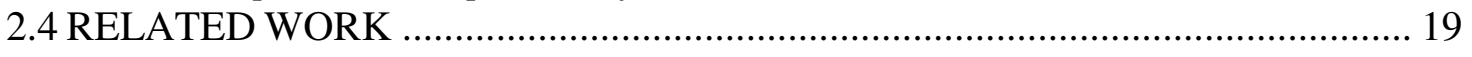

3. CLASSIFICATION OF HISTOLOGICAL MOUSE BRIAN SECTIONS .......... 21

3.1 GENERAL APPROACH............................................................................... 21

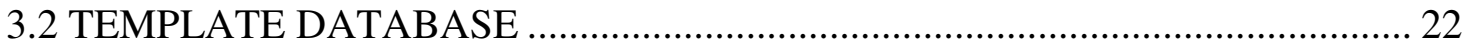

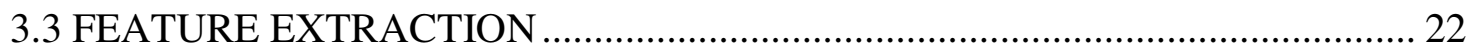

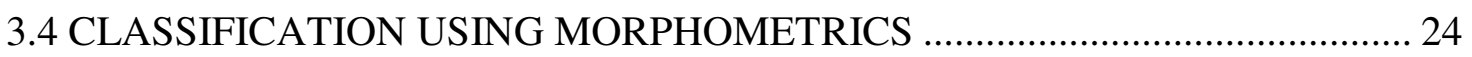

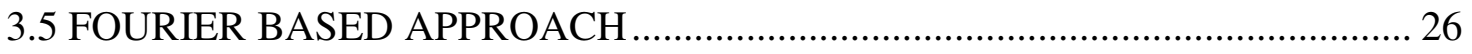

3.5.1 Shape Sampling ............................................................................. 27

3.5.2 2D Fourier Transform ........................................................................ 27

3.5.3 Discrete Fourier Transform on Shape Signatures............................................ 29

3.6 RADIAL DISTRIBUTION BASED APPROACH ……….................................. 30

3.6.1 Centroid-Radii Model .................................................................................... 30

3.6.2 Similarity Measurement ........................................................................... 33

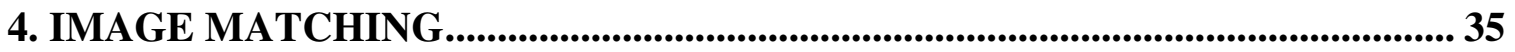

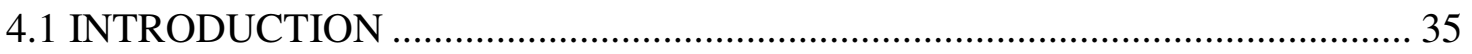

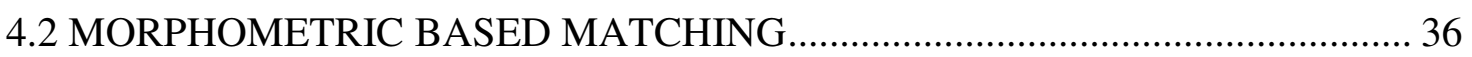

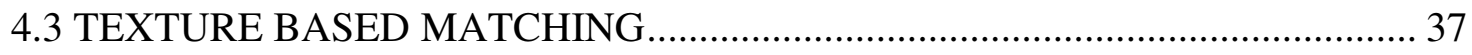

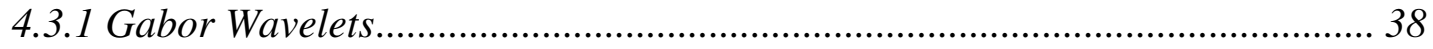




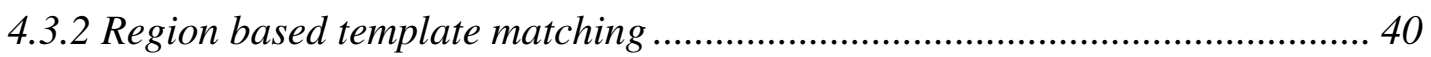

5. EXPERIMENTS AND PERFORMANCE ANALYSIS............................................. 44

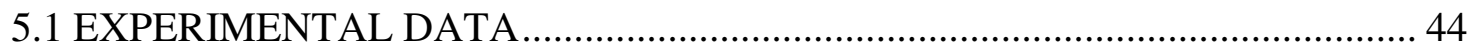

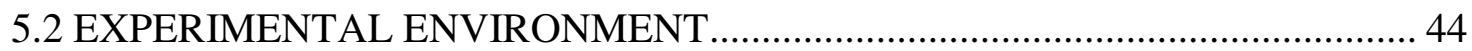

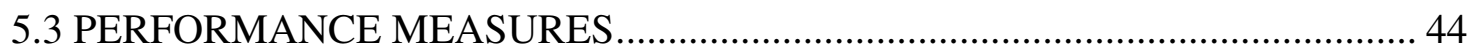

5.5 RESULTS FOR IMAGE MATCHING OF HIPPOCAMPUS IMAGES ............... 46

5.6 RESULTS OF DUAL STAGE IMAGE MATCHING SYSTEM........................... 46

6. CONCLUSION AND FUTURE WORK .................................................................... 49

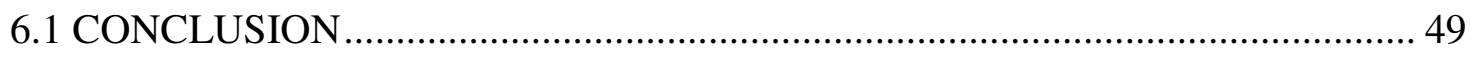

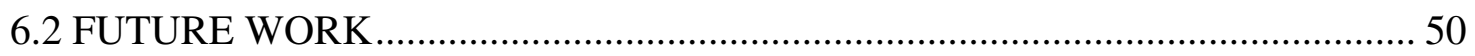

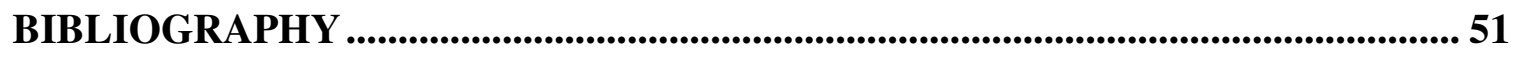

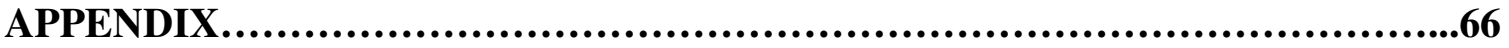




\section{LIST OF FIGURES}

Figure 1.1 Block Diagram of Image Matching System..............................

Figure 2.1 High Resolution Histological Mouse Brain Images.......................6

Figure 2.2 Mouse Brain Image before sectioning................................

Figure 2.3 Photos of the sectioning unit...........................................

Figure 2.4 Sections of Mouse Brain.........................................

Figure 2.5 Classification of Shape Representation Techniques........................15

Figure 3.1 Block Diagram of Dual Stage Approach................................23

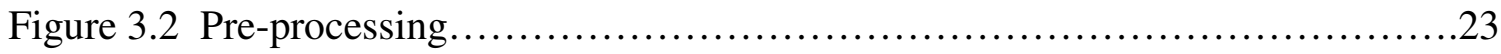

Figure 3.3 Variation of Area, Height, Width, Ratio of Height/Width...................25

Figure 3.4 Classification of Mouse Brain.......................................25

Figure 3.4 Distance between boundary points and centroid........................26

Figure 4.1 Block Diagram of Dual Stage Approach..................................

Figure 4.2 Landmark Detection.................................................

Figure 4.3 Variation of features with position of the Image..........................37

Figure 4.4: Distinguishing Feature blocks in each image in the hippocampus group.....41

Figure 4.5: Template image and test image with regions of interest..................41

Figure 4.6: Evolution of the Hippocampus size withing Group VI....................42

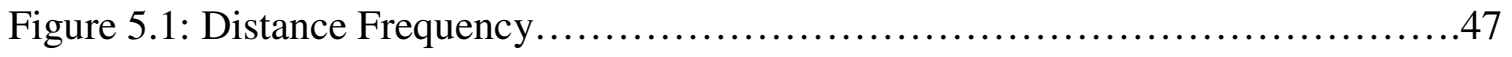

Figure 5.2 Examples of Query Results for Stage II..............................48 


\section{LIST OF TABLES}

Table 3.1 Morphometrics-based Classification................................. 26

Table 3.2 Effect of bin size on performance.................................. 32

Table 5.1 Summary of Experimental Data....................................44

Table 5.2 Comparison of Success Rates of Different Methods........................46

Table 5.3 Success Rates of Ranked Matches......................................46 


\section{INTRODUCTION}

The potential of brain atlases to expedite the dissemination and use of information about brain structure and function is enormous and ever increasing. Numerous efforts are now underway to further develop the technologies that are necessary to support this revolution. Each effort has its own approach and solves some of the complex problems that are associated with creating and retrieving images from a database. This research deals with challenges that are faced by the developers and users of digital atlases of the brain.

\subsection{MOTIVATION}

A common biomedical problem is mapping newly acquired experimental datasets onto a reference atlas. Given a digital brain atlas and a query image of a brain section, the brain mapping problem is to locate the position of the given brain section on the atlas. In this work, we consider 2-D brain atlases and hence 2-D image sections of the brain. The main challenge to current efforts in mouse brain mapping is dealing with the diverse data sets that come from multiple individuals. Diversity can come in the form of file formats as well as in the data quality or comprehensiveness. A commonly used approach in mouse brain research is the manual delineation of regions-of-interest (ROI's). These ROI's are used to derive quantitative values at a macro anatomical level of resolution. The process of delineation involves two images. An image of the stained section differentiates between the gray and white matters and guides delineation of the neuroanatomical boundaries. These ROI's are then projected onto a second image showing the distribution of the compound of interest [1].

The speed and efficiency of the analysis can be improved by eliminating manual ROI delineation. For this purpose, an atlas bearing a set of standard ROI templates is desirable. Aligning an experimental image with the atlas would position the atlas ROI's appropriately. In addition to establishing homology at the level of anatomical structures, registration can be used directly to address equivalent points across brains. This approach has been developed for human neuroimaging [2], [3]. Since the interbrain variability is observed to be low in inbred laboratory animals, this approach can prove to be effective 
in the setting of animal research [4]-[7]. Prior to the alignment, this approach requires locating the position of the given brain image on the brain axis.

In this thesis, we view the brain mapping problem as an image matching problem and developed an automated matching system which is guided by external contours of the sections. This system can also be used to facilitate the development of methods for studying brain shape among different populations, such as mice [8].

\subsection{OBJECTIVES}

The query mouse brain images can be matched with atlas images using either textual information or content-based information. Image content can be described using primitive features such as color, texture, shape or their combination. This research adopts the content based image retrieval (CBIR) approach, which involves indexing and retrieving an image by their content. Specifically, the research focuses on image retrieval using shape features. The main aim of this research is to study and develop promising shape descriptors for retrieval of histological images.

Generally, there are two types of approaches in shape representation. One is the contour based shape method and the other is the region-based method. The main objectives of the research are:

1. To study and compare contour based shape descriptors in analysis of mouse brain sections

2. To develop an automated image matching system (AIMS) for automatic matching of histological mouse brain sections

The techniques investigated and developed in this research can either be directly applied to particular applications such as trademark retrieval, object recognition etc. or be incorporated into any CBIR system to facilitate retrieval using combined image features. For this application, the atlas image, which is most similar to the query image, is obtained by matching the higher-level image characteristics based on the shape information. In our specific case, we used the Paxinos Atlas [14], which contains mouse brain images from the rostral end to the caudal end. 


\subsection{AUTOMATED MOUSE BRAIN MATCHING SYSTEM}

The Automated Image Matching System (AIMS) is fed with the histological mouse brain section to retrieve the corresponding brain section in the atlas. As part of its output, it also produces the approximate Bregma position of the given query image in the brain. The AIMS consists of three components: Image Database templates (templates from the Atlas), Image Classification System and Region based Template matching. The block diagram in Figure 1.1 illustrates the matching of a query image with the images in the database.

The query image is processed through two layers - enhancement and normalization. The enhancement module improves the quality of the input histological image by defining the boundary of the given mouse brain section which leads to proper classification.

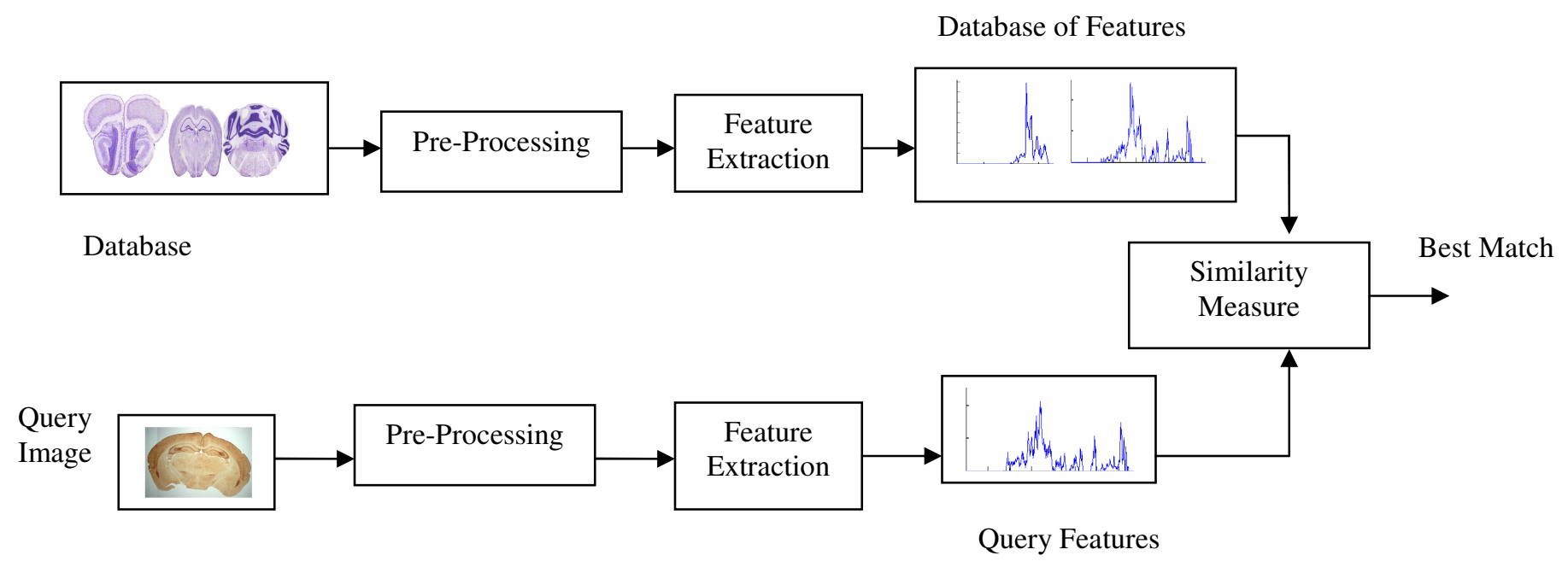

Figure 1.1 Block Diagram of Image Matching System

The output of the image classification system is the class or group to which the given image belongs. This can be achieved using various shape descriptors. In this thesis, three types of shape descriptors for classifying the query image: image morphometrics, Fourier Descriptors, and Radius Distribution. After obtaining the group to which the given image belongs, the problem reduces to finding the best match within the group. 
In this thesis, we develop techniques for the matching of subject image with standard atlas images using region-based template matching. Given the volume of data typically involved, efficiency in the matching becomes a mandatory consideration. In this work, 'subject' image and 'query' image are used interchangeably.

\subsection{THESIS OUTLINE}

This research addresses the problem of matching mouse brain images with a standard Atlas. Chapter 1 describes the motivation and objectives of this research. Contributions of this research have also been summarized.

Chapter 2 provides a background to NeuroInformatics and a review of work related to current mouse brain research. This chapter also includes the Image acquisition system and the required level of robustness in the developed algorithms.

In Chapter 3, three important contour shape descriptors are studied in detail. First, different morphometric based techniques are studied and evaluated. A new shape signature is then proposed based on the radial distributions.

In Chapter 4, a new region based template matching technique is proposed. In this method, Gabor filters are used to extract features from different regions of the image. These features are compared with the features of the template region and the similar images are retrieved.

In Chapter 5, the proposed techniques are applied to histological image retrieval to test the robustness to different shapes and textures. Comparative performance with splinebased matching on histological image retrieval is reported.

Chapter 6 concludes the thesis. Recommendations for future research are also provided. 


\section{BACKGROUND AND RELATED WORK}

\subsection{BACKGROUND}

By far the most intriguing, yet, the least understood anatomical structure is the brain. Understanding how the brain functions will shed light on the fight against various neurogenetic diseases. The development and functionality of the mammalian brain has been studied by systematically collecting a large number of gene expression patterns throughout the mouse brain. Associating specific gene activity with specific functional locations in the brain anatomy results in a greater understanding of the role of the gene's products $[9,10]$. To perform such an association for a large amount of data, reliable automated methods that characterize the distribution of gene expression patterns in relation to a standard anatomical model of the brain are required.

For this reason, building a digital atlas of the brain has become particularly important, as the analysis of brain images often hold the key to brain science. In the past few decades, biological researchers around the world have created brain atlases of various animals such as flies [11], monkeys [12], rats [13] - [15] and mice [3, 7, 8, 9, 16].

\subsubsection{Mouse Brain Atlas}

An atlas represents the average shape of an anatomical structure, and serves as a road map for analyzing images from diverse species and individuals. Research neuroscientists use mouse brain atlases to solve the issues of capture and integration of multidisciplinary data, of 3D reconstruction from serial slices, and of data dissemination, in the expectation that the lessons learned will become applicable directly or indirectly to the human brain. Some of the major applications of digital brain atlases are surgical planning, modeldriven segmentation, education and training [18].

Recent years have witnessed the fast growth in medical imaging methods which are applied in biology and medical science resulting in huge amounts of data imaged from experimental animals. This data plays an important role in the study of animal functions and diseases. If these images could be analyzed automatically, it would help neuroscientists to study the brain anatomy in a fast and efficient way. With rapid 
advancements in Neuroanatomy, there is a need to build a search engine to retrieve similar images from the brain atlas.

\subsubsection{Current Research}

There is a rapidly growing need for brain models which are comprehensive enough to represent brain structure and function across different age, gender, and disease states. One of the important current goals in neuroimaging is to develop procedures for combining functional and anatomical data derived from different individuals [19]. This is necessary to study the nervous system of laboratory animals such as rats. Various histological techniques such as in situ hybridization [20], receptor autoradiography [21], and immunohistochemistry [20], are used in the study of these animals.

Research is going on to develop fast and efficient image registration techniques for histological images [22]. After registration, the next step is to detect the neurons in the high resolution image of the mouse brain as shown in figure 2.1. The resulting annotation will help scientists interpret gene expression patterns more rapidly and accurately. The image below shows the positions of neurons in histological images.
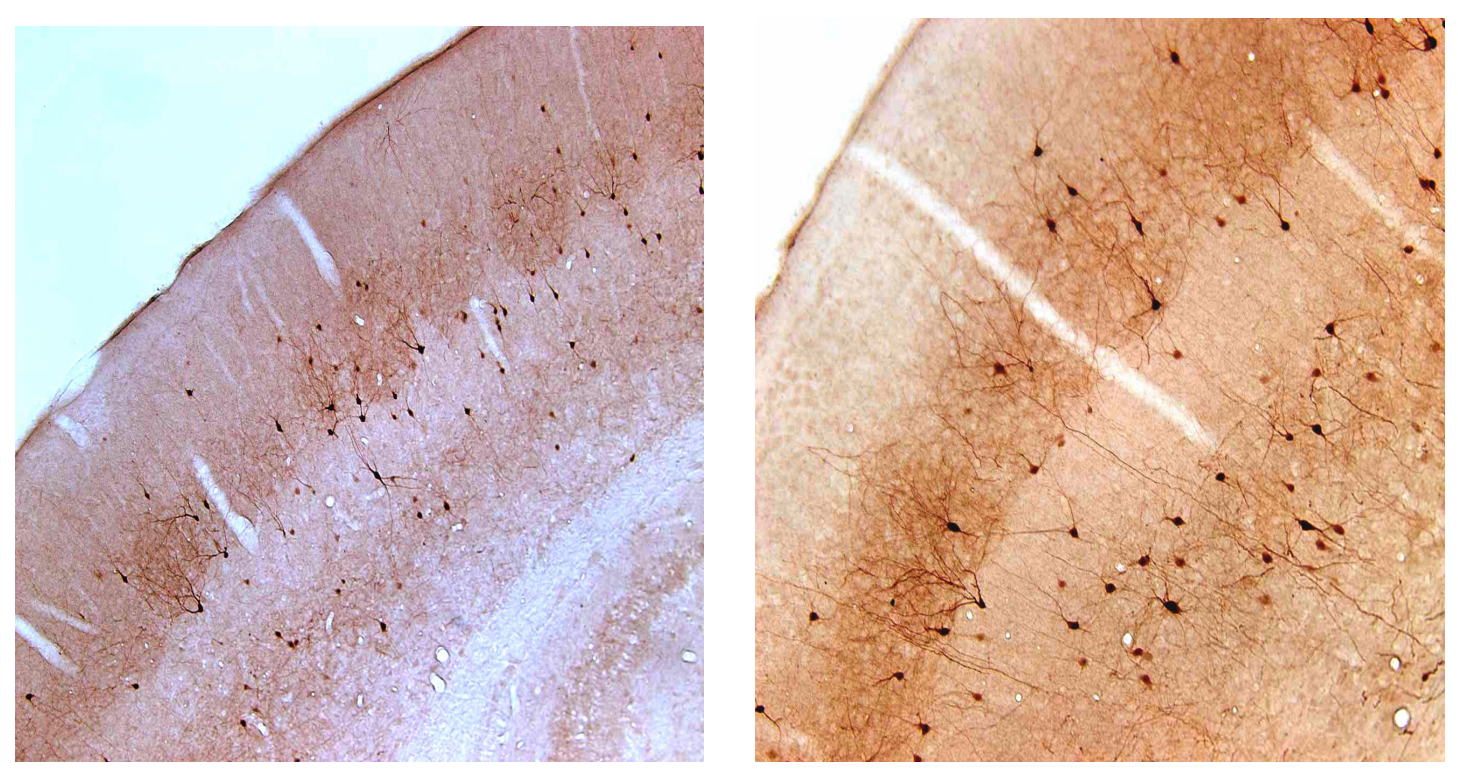

Figure 2.1 High Resolution Histological Mouse Brain Images. Images of sections from transgenic mouse brains, line X94 by Dr. A. Agmon. See Ref. 65 
The Laboratory of Neuro Imaging Resource (LONIR) at University of California, Los Angeles (http://www.loni.ucla.edu/NCRR/) develops new techniques to investigate brain structure and function in a multidimensional complexity. Various other research centers such as Biomedical Informatics Research Network (BIRN) [63], Neuroimaging Analysis center (NAC) [64] at Boston, MA are working on developing image processing and analysis techniques for basic and clinical neurosciences. This includes development of algorithms and techniques for post processing of imaging data, new segmentation techniques which help in identification of brain structures and disease, visualization tools to display complex anatomical and quantitative information, developing digital anatomy atlases to support both interactive and algorithmic computational tools.

\subsubsection{Data Collection}

In medical imaging, volumetric data generated by 3D imaging methods such as MRI and CT have wide ranging applications in the visualization and analysis of organs. 2D imaging methods, such as optical microscopy, typically generate serial sections with much higher resolution than MRI or CT scans. The following sections explain the process of acquiring histological mouse brain sections.

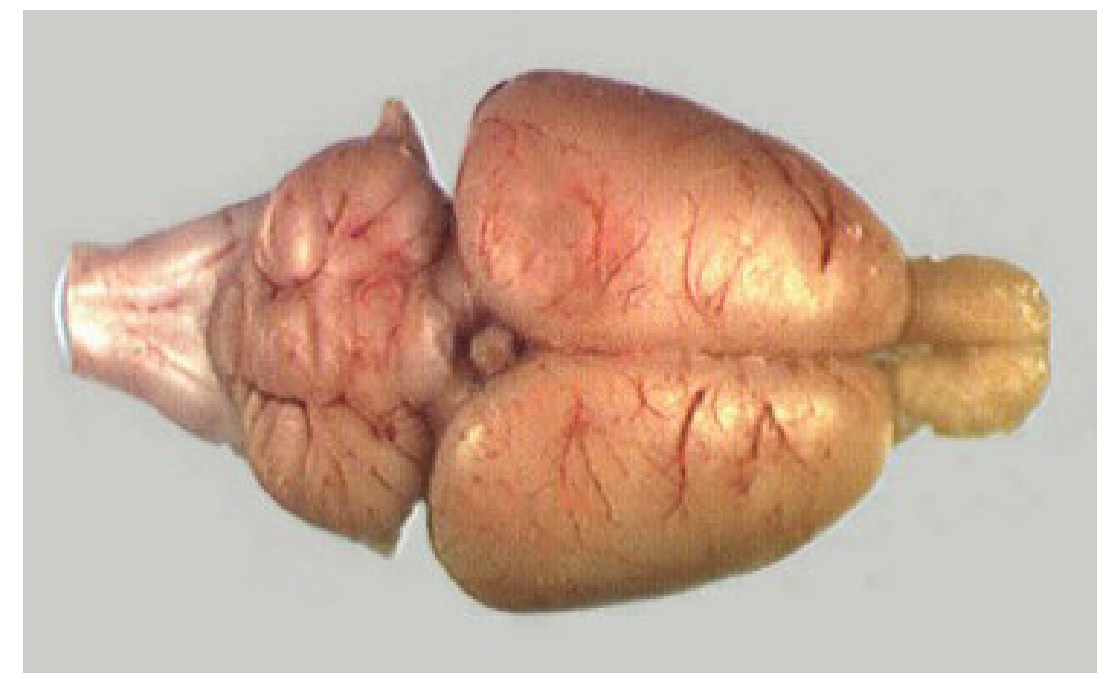

Figure 2.2 Mouse Brain Image before sectioning [23]

\subsubsection{Cryo-sectioning:}

The protocol described in this section has been used by Paxinos Atlas [14]. Brain images are collected on P7 C57BL/6 Mus Musculus Brain [24], [25] using cryo-sectioning. The 
brain is first extracted from the skull and put into solution to freeze. The frozen brain is then cut coronally into serial cryo-sections each $25 \mu \mathrm{m}$ thick. Tissue sections are placed on slides and then a Nissl stain is applied. After staining, a coverslip is applied to each slide. The tissue section is then imaged using light microscopy at the resolution of $3.3 \mu \mathrm{m}$ per pixel. Given that the mouse brain is roughly $0.6 \mathrm{~cm} \times 0.8 \mathrm{~cm} \times 1.2 \mathrm{~cm}$, imaging a single mouse brain yields around 500 images whose dimensions are 2000 by 3500 pixels. The dataset $\mathrm{I}$ as described in Chapter 5 is derived from Dr. Gregor Eichele's laboratory at Baylor College of Medicine for high throughput in situ hybridization. An image of the sectioning unit and the cutting unit is shown in Figure 2.3.

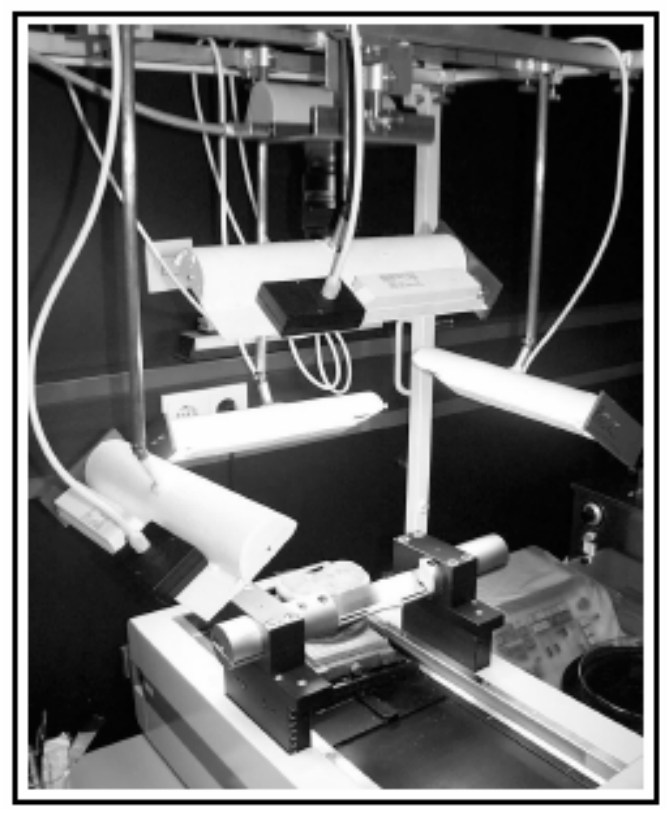

(a) Sectioning of a Brain

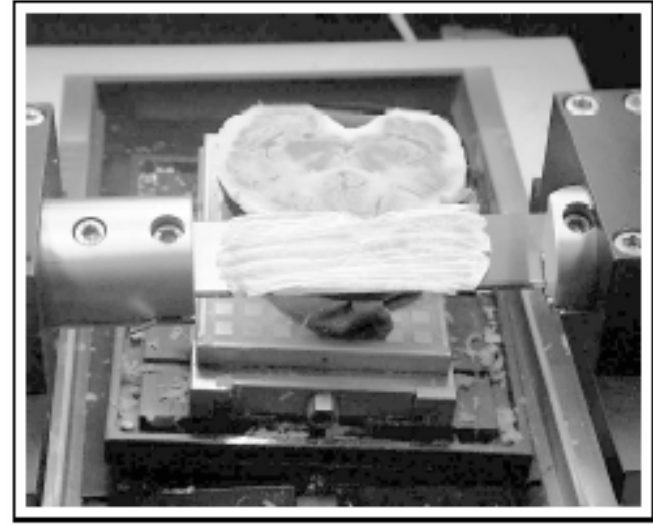

(b) Details of the Cutting unit

Figure 2.3 Photos of the sectioning unit [26]

\subsubsection{Image Acquisition:}

The following protocol is the one used in the Dr. Agmon's lab at WVUHSC to stain brain sections from transgenic mice, such as the sections illustrated in Figure 2.1. This protocol is used to visualize neurons containing green fluorescent protein in brains from transgenic mice. To obtain the brain sections, mice were deeply anesthetized with $4 \%$ chloral hydrate $(0.02 \mathrm{ml} / \mathrm{g}$ i.p.) and perfused through the ascending aorta with $10 \mathrm{ml}$ of saline, followed by $35 \mathrm{ml}$ of $4 \%$ paraformaldehyde in $0.12 \mathrm{M}$ sodium phosphate buffer ( $\mathrm{PB}), \mathrm{pH}$ 7.3 , at a rate of $5 \mathrm{ml} / \mathrm{min}$, using a peristaltic pump. Fixed brains were dissected and post- 
fixed for $4 \mathrm{hrs}$ at $4^{\circ} \mathrm{C}$, then placed overnight in $30 \%$ sucrose in $0.075 \mathrm{M}$ PB at $4^{\circ} \mathrm{C}$ for cryoprotection. Equilibrated brains were sectioned on a freezing microtome (micron) into $40 \mu \mathrm{m}$ thick sections, in coronal planes, and sections rinsed three times in $0.5 \mathrm{M}$ Tris buffer (Tris), $\mathrm{pH} 7.6$, at room temperature.

To quench endogenous peroxidases, sections were washed for 20min in 3\% $\mathrm{H} 2 \mathrm{O} 2$ in $10 \%$ Methanol (Sigma), then rinsed three times in Tris and transferred into blocking solution (5\% normal goat serum (NGS) $+0.5 \%$ Triton-X100 (TX) in Tris). Sections were transferred directly from blocking solution into diluted primary antibody (1:1000 rabbit anti-GFP, Molecular Probes) in vehicle (1\% NGS+0.1\% TX in Tris), and gently agitated for $36 \mathrm{hrs}$ at $4^{\circ} \mathrm{C}$. After three washes, in Tris, sections were incubated in ABC solution (Vector) for $1 \mathrm{hr}$ at room temperature, rinsed three times in Tris, and placed 3-5mins into $0.05 \%$ 3,3'-diaminobenzidine tetrahydrochloride (DAB, Aldrich)+ $0.01 \% \mathrm{H} 2 \mathrm{O} 2$ in Tris. Reaction was stopped with cold buffer, and sections rinsed three times and mounted from $40 \%$ gelatin-alcohol onto glass slides, air-dried overnight, and coverslipped in a drop of Cytoseal (Richard-Allan Scientific). DAB-labeled sections were imaged on an Olympus Provis AX70 microscope equipped with a Magnafire camera. The position of images sectioned is as shown in the figure 2.4. The markers indicated in the figure are spaced $1 \mathrm{~mm}$ apart and correspond to levels of the atlas $[6,14]$. The zero coordinate is called the Bregma point.

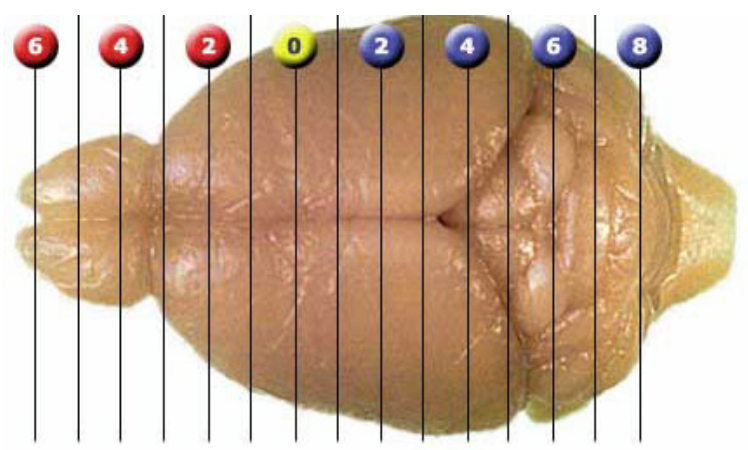

Figure 2.4 Sections of Mouse Brain [27]

During image acquisition, each slice may exhibit tissue distortions of the following sort:

- Global rotations and translations introduced when the tissue sections are placed on the slides. 
- Regional tissue deformations in the form of vertical stretching and compacting induced both during cutting and when the section is placed onto the slide.

- Extreme deformations which lead to tearing and folding.

- Image artifacts, such as dust (dark artifact) and air bubbles (black ring-shaped artifact), introduced during cover slipping.

In this thesis, we could address the problem of rotations and translations during image acquisitions. The techniques developed for image classification and matching are invariant to scale, rotation and translation.

\subsection{ELEMENTS OF IMAGE MAPPING}

The image mapping technique is determined depending on the type of images, their distortions, modalities, conditions under which images are acquired, types of sensors used for acquisition of images and the application. The image mapping technique is generally specified using two components: feature extraction and similarity measure. These characteristics are explained in detail in the following sections.

\subsubsection{Feature Extraction:}

The primary task of an image mapping system is to determine a form of representation for the images to be mapped. This is performed by extracting some quantitative features from the image. The result is the "feature space" which provides a description of relevant information in the images that will be used for matching. In content based image retrieval, the primary features used to distinguish between images are based on shape, color, texture and motion. The primary features that have been used in image matching are edges and contours [33], image intensity, fourier descriptors (FD's) [32, 35, 38], statistical features-moment invariants [42, 43], centroid axes [31], structural and syntactic features [42].

Given the significance of contour-based methods and Fourier descriptors, boundary information of objects in an image obtained by using edge detectors or contour extraction methods represents an intrinsic structure of the image and is less sensitive to noise [28]. Boundary information is invariant to illumination conditions, effect of using different 
sensors as well as multi-spectral conditions whereas the intensity values of the images are not preserved in such conditions. A chain code representation technique was suggested in [31] by Wang et al. In this method, a contour is traced in a fixed direction from fixed starting point which represents a shape contour.

Lu and Zhang in [29], [35] suggested two methods for shape retrieval using Fourier descriptors. The first method is based on Fourier descriptors with centroid distances and curvature function. Fourier descriptors are the normalized coefficients of the Fourier transformation applied to a signal derived from samples from the contour, such as the boundary coordinates. Experiments have shown that the centroid distance function, the distance from the contour to the centroid gives better performance than other types of signals.For developing a robust technique that can locate local shape features efficiently, Lu et al. introduced their second method in which short-time Fourier Descriptors (SFD's) are used for shape retrieval. They have made a comparative study using two descriptors and observed that though SFD's perform better for certain shapes such as butterfly and tree, FD's proved to be more efficient way of representing shape.

Kauppinen et al. [32] conducted another experimental comparison, by in which the performance of Autoregressive modeling for 2D shape classification is compared with that of Fourier-based descriptors. It has been reported that fourier descriptors perform better even in noisy conditions. A different approach for describing contours for shape retrieval was suggested in [30] by Cohen et al. Here, B-splines are used to model the contours since they offer local controllability and shape invariance under affine transformation. Other commonly used techniques for shape retrieval include Hidden Markov models [33], shape contexts [34], geometric hashing [36] etc.

In this problem of mouse brain matching, using the shape of the section solves the matching problem but does not yield very high performance in terms of accuracy. Instead, image texture which relates to the inherent nature of the images leads to accurate image matching. The feature space that represents the image information is detected separately for the images and compared with those in the database. Knowledge of the imaging system and the sources of variations play a key role in determining the best set 
of feature to direct the search for accurate mapping.

\section{$\underline{\text { 2.2.2 Similarity Measure: }}$}

Shape similarity measurement requires objective measures of the extent to which it can be determined whether a given shape A is more similar to another shape B or to shape C. Shape similarity measurement is fundamental to classifying shapes, and the particular method used is generally dependent on the specific problem. For instance, in a situation where size is an important parameter, two shapes with similar areas can be more similar to each other than two shapes with significantly different areas. Therefore, the shape features adopted for their characterization play a significant role with respect to defining similarity between the given shapes. Some of the commonly employed similarity measures for image matching are correlation coefficients [54], Phase correlation [56], contour/surface differences [59], sum of absolute differences in intensity/contours [58].

Some of the properties of a shape similarity measure are discussed here. A shape dissimilarity measure, or distance function, on a set of shapes $S$ is a function $d: S \times S \rightarrow$ R. The following conditions apply to all the shapes $A, B$, or $C$ in $S$.
a. Nonnegativity:
$d(A, B) \geq 0$.
b. Identity:
$d(A, A)=0$ for all shapes $A$.
c. Uniqueness:
$d(A, B)=0$ implies $A=B$.
d. Strong triangle inequality:
$d(A, B)+d(A, C) \geq d(B, C)$.
e. Triangle inequality:
$d(A, B)+d(B, C) \geq d(A, C)$.
f. Symmetry:
$d(A, B)=d(B, A)$.

g. Invariance: $d$ is invariant under a chosen group of transformations $G$ if for all $g \epsilon$ $G, d(g(A), g(B))=d(A, B)$.

h. Noise robustness

For many object recognition problems, the similarity measure has to be invariant under affine transformations. In contour-based shape representations, noise is considered as an extra region attached to any location on the contour, and hence defines robustness similarly. Similarity metrics operate on both reference and query images and thus reduce the noise during this stage resulting in definite detection of features. These features 
combined with an efficient similarity metric can reduce the search space for possible matches. Using the sum of absolute differences of intensity [58] and sum of absolute differences of contours [59] for matching the images with no local distortions, efficient computation is achieved. Among similarity metrics mentioned above, cross-correlation is robust for images with white noise. Phase correlation measures are used for frequencydependent noise and the noise generated due to varied lighting conditions.

\subsection{SHAPE BASED IMAGE MATCHING}

Shape is one of the most important low level image features in the Content Based Image Retrieval (CBIR). Applications on Shape retrieval can be found in many areas, such as meteorology, medicine, space exploration, manufacturing, entertainment, education, law enforcement and defense. Shape retrieval involves three primary issues: shape representation, shape matching/shape similarity measure, and shape indexing. Shape representation and similarity measure are key issues in robust shape retrieval. Shape indexing and efficient shape matching are important for fast retrieval and short response time to user queries. Evidently, the representation scheme adopted also could have a significant impact on both robustness and efficiency.

\subsubsection{Shape Representation}

An effective and compact shape representation system is a critical element for various computer vision applications. Boundary data is extracted as $(x, y)$ coordinates in the image space and has to be represented in a form suitable for indexing, and similarity matching. Using such shape representation methods, the $2 \mathrm{D}$ representation of a boundary, i.e., the $(x, y)$ coordinates of boundary points, has to be converted into a form that has the following properties [37]:

- Invariance: two boundaries with same shape should have the same representation.

- Compact: Redundancies are to be removed by capturing similarities, region information, local deformations, and dividing object into sub-regions through parameter setting.

- Efficiency: the representation should be computationally efficient with respect to time and space. 
- Ease of implementation: the representation should be the less complex

- Computation of shape properties: the representation should support computation of shape properties such as symmetry, area, perimeter, etc.

- Meaningful representation: The representation should retain properties of the shape that are meaningful to the application.

Shape representation algorithms used for medical images have to obtain reduced feature dimensionality while retaining the important biomedical features. Some of the properties which are more relevant to biomedical image retrieval include:

- Geometric Invariance: the features should be invariant to rotation, translation, and scaling.

- Compact representation: essential shape information should be maintained in a significantly reduced manner.

- Fast matching speed: efficient shape feature computation and fast retrieval in a large image database.

- High quality image retrieval: retrieved images should be relevant to the query.

The variety of shape representation techniques in the literature are first classified into two categories: Contour-based methods or Region-based methods, depending on whether the shape features are extracted from the contour or the whole region. Under each class, different methods are further classified into Structural and Global based on whether the shape is represented as a whole or in parts (primitives). Figure 2.5 shows this general classification of popular shape representation schemes.

\subsubsection{Contour-Based Shape Representation Techniques:}

Contour shape techniques exploit shape boundary information. There are generally two types of approaches for contour shape modeling: continuous approach (global) and discrete approach (structural). In Continuous approaches, the feature vector derived from the integral boundary is used to describe the shape. The measure of shape similarity is either point-based matching or feature-based matching. Discrete approaches divide the shape boundary into segments, called primitives using a particular criterion. The final 
representation is usually a string or a graph (or tree). the similarity measure is then based on string matching or graph matching.

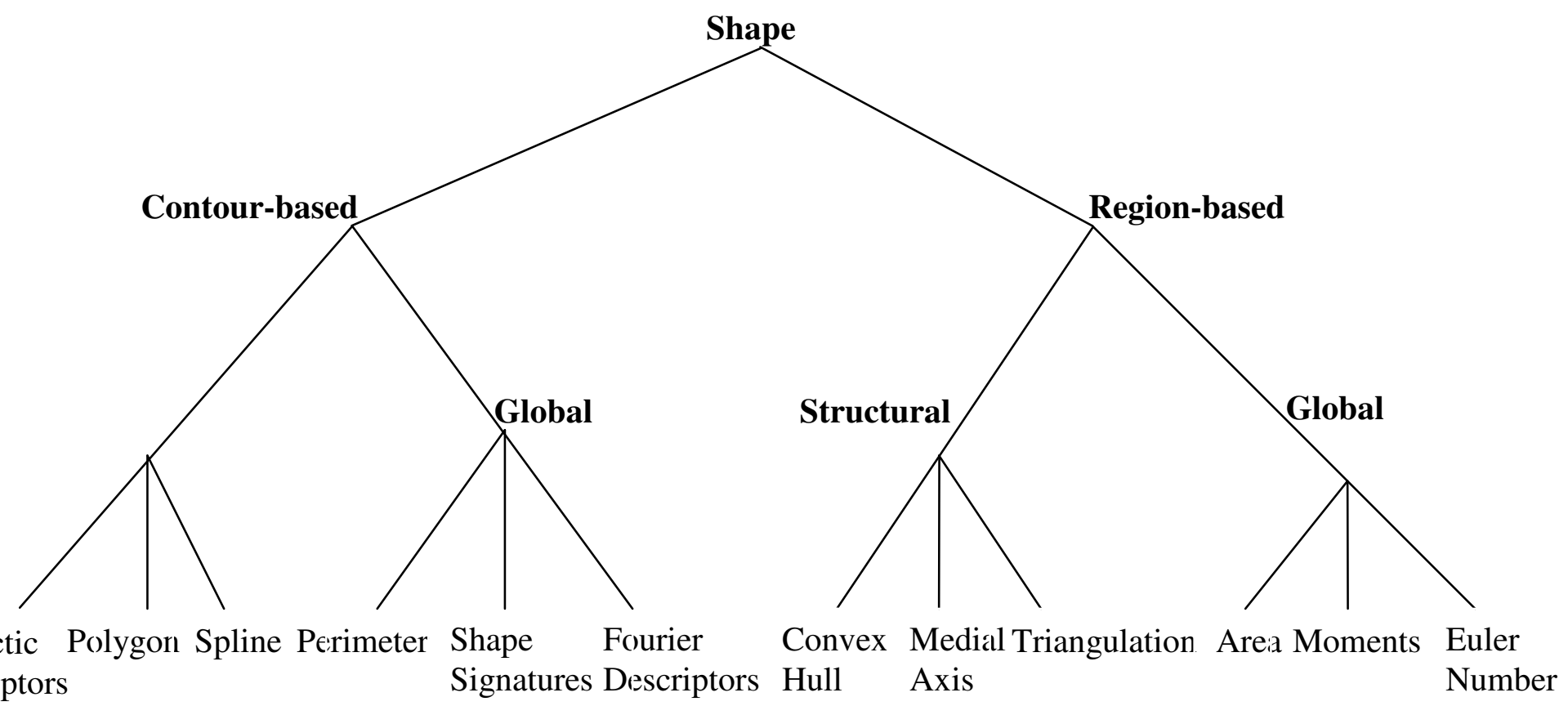

Figure 2.5 Classification of Shape Representation Techniques

Common simple global descriptors are area, circularity (perimeter2/area), eccentricity (length of major axis/length of minor axis), major axis orientation, and bending energy [39]. These simple global descriptors usually can only discriminate shapes with large dissimilarities. Therefore, they are usually used as filters to eliminate false hits or combined with other shape descriptors to discriminate shapes.

Shape signatures represent shapes using a one dimensional function derived from shape boundary points. Many shape signatures exist, including complex coordinates, polar coordinates, central distance, tangent angle, cumulative angle, curvature, area and chord-length. Shape signatures have been extensively studied in [48, 29]. Shape signatures are usually normalized into being translation and scale invariant. Shape signatures reduce shape matching from 2-D space into 1-D space. However, in order to compensate for orientation changes, the shift matching is needed to find the best matching between two shapes. Alternatively, signature can be quantized into a signature 
histogram, which is rotation invariant and can be used for matching [47].

Another member in the shape analysis family is structural shape representation. With the structural approach, shapes are broken down into boundary segments called primitives. Structural methods differ in the selection of primitives and the organization of the

primitives for shape representation. Common methods of boundary decomposition are based on polygonal approximation, curvature decomposition and curve fitting [44, 45, 46]. The result is encoded into a string of the general form:

$$
S=S_{1}, S_{2}, S_{3}, \ldots \ldots \ldots S_{n}
$$

where $S_{i}$ may be an element of a chain code, a side of a polygon, a quadratic arc, a spline, etc. $S_{i}$ may contain a number of attributes such as length, average curvature, maximal curvature, bending energy, orientation etc. The string can be directly used for description or can be used as input to a higher level syntactic analyzer.

\subsubsection{Region-Based Shape Representation Techniques:}

In region based methods, all the pixels within a shape region are taken into account to obtain the shape representation. Common region based methods use moment descriptors to describe shape. Other region based methods include the grid method, shape matrix, convex hull and media axis. Hu published the first significant paper on the use of image moment invariants for two-dimensional pattern recognition applications [40].

Moment methods extract the statistical distribution of region pixels. The lower order moments or moment invariants carry physical meanings associated with region pixel distribution. Using nonlinear combinations of the lower order moments, a set of moment invariants [41] (usually called geometric moment), which has the desirable properties of being invariant under translation, scaling and rotation, are derived. Among the many moment shape descriptors, Zernike moments are the most desirable for shape description. The Zernike moment descriptor has been adopted by MPEG-7 as a region-based shape descriptor [42], [43]. Region based methods are usually more robust and application independent. However, they usually require more computation and more storage than contour-based descriptors. 


\subsubsection{Shape Similarity Measures:}

Some of the commonly used Shape Similariy measures are Euclidean distance, Procrustes distance [52], Bookstein test, Kendall test [51], Hausdorff distance [53], $\chi^{2}$-metric, Kolomogorov-Smirnov distance, etc. The similarity measure of two shapes can be defined as the mathematical distance between the feature vectors for the two shapes. It can be viewed as for a given query $S_{Q}$, find the image in the database $S$ within the distance $d$ of $Q$, i.e retrieve all images in $\mathrm{S}$ such that $\left(S_{i}, S_{Q}\right)<d$, where $\mathrm{d}$ is the distance being measured.

Euclidean Distance: It may be defined as shown in Equation 2.7, where $d$ is the distance between shapes $A$ and $B$, and $a_{i}, b_{i}$ and are feature vector elements. The feature vectors are of length $n$.

$$
\|d\|_{2}=\sqrt{\sum_{i=1}^{n}\left|a_{i}-b_{i}\right|^{2}}
$$

Hausdorff Distance: It can be defined as the maximum distance of a set to the nearest point in the other set. In mathematical terms, hausdorff distance from set A to set B is a maximin function, defined as

$$
\mathrm{H}(\mathrm{A}, \mathrm{B})=\max _{a \varepsilon A}\left\{\min _{b \varepsilon B}\{d(a, b)\}\right\}
$$

where $\mathrm{a}$ and $\mathrm{b}$ are points of sets $\mathrm{A}$ and $\mathrm{B}$ respectively, and $\mathrm{d}(\mathrm{a}, \mathrm{b})$ is any metric between these points. The most commonly used metric employed here is the Euclidian distance. Hausdorff distance is used to find similarity measure between polygons.

Procrustes Distance: In [52], this shape metric has been used to align shapes. Procrustes distance can be mathematically defined as the sum of the squared point distances after the alignment. The distance $\mathrm{d}$ between two shapes $\mathrm{x} 1$ and $\mathrm{x} 2$ can be calculated using equation 2.9.

$$
P_{d}^{2}=\sum_{j=1}^{n}\left[\left(x_{j_{i}}-x_{j_{2}}\right)^{2}+\left(y_{j_{i}}-y_{j_{2}}\right)^{2}\right]
$$


Procrustes distance can be calculated in four steps: computation of centroid, resizing each shape to be of same size, aligning the two shapes along their position at the centroids, aligning the shapes with respect to the orientation by rotating to required degree. To calculate centroid of the given shape, we use equation 2.10.

$$
(\bar{x}, \bar{y})=\left(\frac{1}{n} \sum_{j=1}^{n} x_{j}, \frac{1}{n} \sum_{j=1}^{n} y_{j}\right)
$$

A shape size metric is used to align the shapes. A commonly used shape size metric is the centroid distance. It can be stated as:

$$
S(x)=\sqrt{\sum_{j=1}^{n}\left[\left(x_{j}-\bar{x}\right)^{2}+\left(y_{j}-\bar{y}\right)^{2}\right]}
$$

\subsubsection{Shape Classification:}

After shape processing, representation, and characterization involving feature extraction, classification algorithms are usually applied in order to assign a class to each considered shape. There are two particularly important aspects related to shape classification. The first is the problem of, given an input shape, deciding whether it belongs to some specific predefined class. This can also be thought of as a shape recognition problem, usually known as supervised classification. The second equally important aspect of shape classification is how to define or identify the involved classes in a population of previously unclassified shapes. This represents a difficult task, and expert knowledge acquisition problems are also involved. The latter situation is known as unsupervised classification or clustering. Both supervised and unsupervised classification involve comparing shapes i.e., deciding how similar two shapes are, by matching specially important corresponding points (typically landmarks or salient points) on the shapes.

The first stage of Automatic Image Matching System involves image classification which is a supervised classification since the classes are pre-defined by experts based on shape and other unique features of the images. Some of the applications for invariant 2D shape recognition include industrial inspection, classification of chromosomes, target 
recognition and scene analysis.

\subsubsection{Unsupervised Shape Classification:}

As the name suggests, in this type of classification, the classes are not pre-defined. Therefore, it is important to devise methods that attempt to find shape classes based only on the unclassified pattern data, an approach that is commonly known as unsupervised learning. The identification of data clusters in the data sets is an ordinary way of defining shape classes, and this is done using clustering algorithms [57].

\subsubsection{Supervised Shape Classification:}

When the shape classes are predefined, or examples are available for each class as in our case, it is often desirable to create algorithms that take a shape as input and assign it to one of the classes, i.e., it recognizes the input shape. In this thesis, we perform a supervised classification. The mouse brain images are classified into 11 groups based on the shape and internal features. Therefore, when a query image is given, the class to which it belongs to can be identified.

\subsection{RELATED WORK}

In our work, we focused on the task of mapping histological mouse brain images on the brain atlas. Apart from being applied in the field of neuroinformatics, other researchers with the help of neuroscientists have developed histological image retrieval techniques for various other applications. These techniques are employed to automatically recognize and retrieve medical images which are used in patient diagnosis, surgical planning, and training. Research has been done in both content based and semantic based histological image retrieval.

Tang and Honka in [62] suggested a technique which integrates both iconic and semantic content of the image for histological image analysis. Other methods to automate the identification of the homologous plane include B-splines based techniques [1]. In this technique, only information from the external contour of the section is used to drive the matching procedure. The contours are represented by B-splines due to shape invariance 
and local controllability properties of B-splines. Curve matching is achieved by using a similarity measure that depends directly on the parameters of the B-spline. After obtaining the contour of the given query image, the corresponding curve $r\left(t^{\prime}\right)$ is represented by $n+1$ knot points.

$$
\hat{r}=\left(\hat{r_{0}}, \hat{r}_{1}, \ldots . \hat{r_{n}}\right)
$$

The template database consists of set of $\mathrm{P}$ different curves $\mathrm{r}_{\mathrm{p}}\left(\mathrm{t}^{\prime}\right), \mathrm{p}=1,2, \ldots . P$. Each curve is represented using $n+1$ knot points.

$$
\hat{r_{p}}=\left(\hat{r_{0}}(p), \hat{r_{1}}(p), \ldots . . \hat{r}_{n}(p)\right)
$$

Therefore, the image matching problem is reduced to the finding the database curve that has minimum distance with the query curve.

$$
\min _{1 \leq p \leq P}\left\{\sum_{i=0}^{n}\left\|\hat{r_{i}}(p)-\hat{r}_{i}^{*}(p)\right\|^{2}\right\}
$$

Since this technique uses only the external contour and does not exploit the internal features of the images for matching, the performance was limited to $73 \%$. To improve the efficiency and speed, we propose a two-stage algorithm in which the first stage is used to classify the given query image using boundary-based shape information and in the second stage, we use the internal texture features within the predicted class to obtain the best match for the given query. 


\section{CLASSIFICATION OF HISTOLOGICAL MOUSE BRAIN SECTIONS}

\subsection{GENERAL APPROACH}

We present a dual-stage approach to the problem of image matching in which we employ shape descriptors and region-based template matching techniques. The two stages of our approach are:

i. Image Classification

ii. Image Matching

In the initial stage, the query image is classified into one of the 11 pre-defined groups using shape signatures. From the output of the first stage it can be determined if the subject image is within the hippocampus region (Group VI). This image is fed to the second stage to determine its exact location in the mouse brain. The output of the second stage is the most similar looking image from the template database. Figure 3.1 shows the flow chart of the Dual Stage Image Matching algorithm.

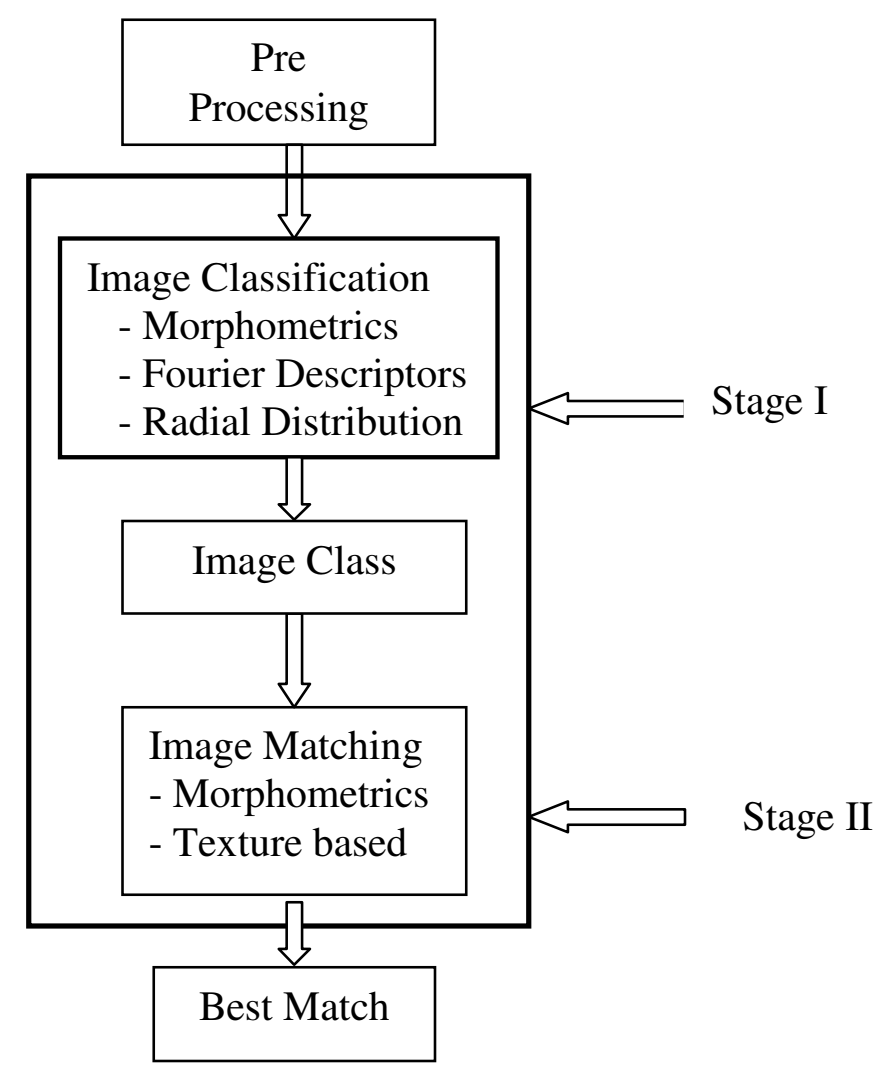

Figure 3.1 Block Diagram of Dual Stage Approach 
The shape representation must be invariant to translation, rotation and scale. In this thesis, we demonstrate the performance of two shape representation techniques: Fourier Descriptors and Radial Distributions on histological mouse brain sections. The performance of morphometrics based image classification has been studied.

\subsection{TEMPLATE DATABASE}

Due to the anatomical homogeneity between members of the same species, comparative studies of data imaged from different individuals belonging to the same species rely on a common template, called an atlas. There are a number of 2D digital mouse brain atlases. As the underpinning for a coordinate system, atlases serve two purposes. First, their demarcation into anatomical regions defines a standard template to be used in classification of experimental sections. Second, features on the atlas, either geometric or gray value, drive the matching process. In this project, we used the Paxinos atlas [16] as the template database. The brain depicted in this atlas is obtained from 26 adult C57BL/J6 mice. This atlas represents the areas of the brain from the olfactory bulb to the middle of the medulla oblongata. The average interplate distance is $120 \mu \mathrm{m}$ and it varies from $40 \mu \mathrm{m}$ and $160 \mu \mathrm{m}$. Alternate sections are stained with cresyl violet or are reacted to reveal aceytylcholinesterase (AChE). These sections are not considered for image mapping. The sections stained with C57BL/J6 are classified into 11 groups based on visual observation under the guidance of a neuroscience expert. Each group represents a structure in the mouse brain. For example, group VI consists of images with hippocampus. The images of the Paxinos atlas and the classes are given in Appendix A.

\subsection{FEATURE EXTRACTION}

Edge detection is the first step in feature extraction. The features extracted describe the image content. Image content to the user is often at the semantic level. For example, the user may be seeking images with cases of "mouse brain", "hippocampus", "Anterior Osteophytes of grade 3", etc. Mapping such a high level query to suitable image characteristics is a challenging task. However, a middle ground is achievable by identifying appropriate image features and representing them in a form to enable responses to similar queries by defining areas in the segmented structures and assigning 
meaning to measurements on those areas. It is thus important to identify the image features to be extracted to enable responses to queries relevant to the class of images in question. The histological mouse brain images are color images containing useful shape and texture information. The shape of the query image is obtained using edge detection techniques.

Edges in an image are located at local maxima of the gradient in the image. They form the boundary between two dissimilar regions in an image. Edges in an image can be detected using high pass filters like Sobel, Canny [60], Prewitts, Roberts etc. The high pass filters retain the sharp details like edges in the image. After experimenting with different filters, the best results were obtained using Canny filter.

The Canny operator works in a multi-stage process. First, the image is smoothed by Gaussian convolution. In the next step, a simple 2-D first derivative is applied to the smoothed image to highlight regions of the image with high first spatial derivatives. Edges give rise to ridges in the gradient magnitude image. The algorithm then tracks along the ridge top and sets to zero all pixels that are not on the ridge top to produce a thin line in the output. This process is known as non-maximal suppression. The tracking process exhibits hysteresis controlled by two thresholds: $T 1$ and $T 2$ with $T 1>T 2$. Tracking can only begin at a point on a ridge higher than $T 1$. Tracking then continues in both directions out from that point until the height of the ridge falls below $T 2$. This hysteresis helps to ensure that noisy edges are not broken up into multiple edge fragments.

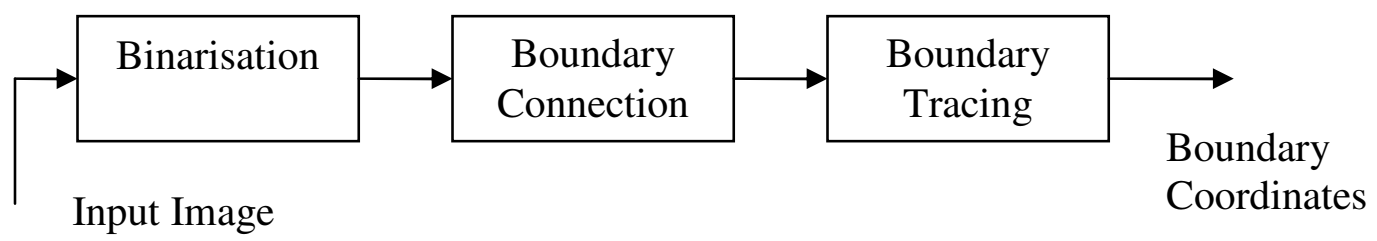

Figure 3.2 Pre-processing

After obtaining the outline shape of the image, it is given as input to the preprocessing stage. The block diagram of the preprocessing is shown in Fig 3.2. The shape images are often corrupted with noise. The shape obtained from edge detection could have noise 
around the shape boundary. A filtering process eliminates those isolated pixels using morphological operations and the gaps between boundary points are filled using an mconnectivity technique. The boundary co-ordinates are obtained by tracing the shape using 8-connectivity contour tracing. These boundary co-ordinates are used to calculate centroid distances, radial distributions, and Fourier descriptors.

\subsection{CLASSIFICATION USING MORPHOMETRICS}

Morphometrics is the branch of mathematics that studies the metrical and statistical properties of shapes and shape changes. It has been used to study shapes of geometric objects like molecules, fossils, brains, bird wings, ancient handcraft, modern cars, etc. We use this technique to study the shape changes of mouse brain sections along the brain axis. We use various measurements to determine the class of the brain such as area of the brain section, area of the bounding box, height and width of the image and a combination of these measurements with different weightage. The parameters involved in this technique are illustrated in the Figure 3.3. The variation of the template images with respect to area, height and width of the brain section is shown in Figure 3.4. The image numbers on the horizontal axis of the plots relate to the image section number as used in the Paxinos Atlas [16]. Also, refer to Figure 3.5 for a visual indication of the numbering scheme.

$\mathrm{R}_{\mathrm{HW}}$ - Ratio of height over width of the brain section

$\mathrm{R}_{\mathrm{A}}$ - Ratio of the area of brain section over the area of the bounding box of the image

$\mathrm{R}_{\mathrm{Hc}}$ - Ratio of height of the image along the centroid over the height of the image

$\mathrm{R}_{\mathrm{C}}-\left[\mathrm{R}_{\mathrm{HW}} * \mathrm{R}_{\mathrm{A}} * \mathrm{R}_{\mathrm{Hc}}\right]^{1 / 3}$

Width (W)

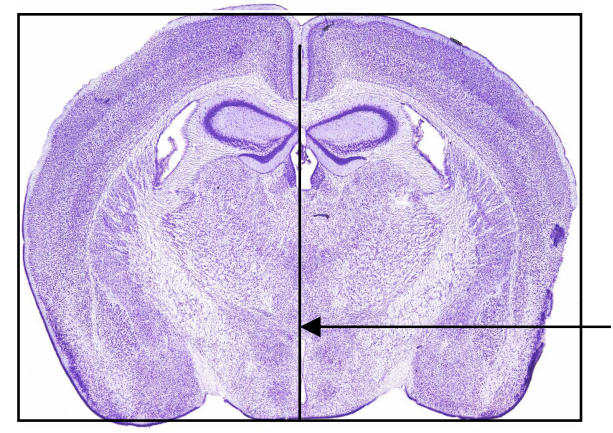

Height $(\mathrm{H})$

Height (Hr)

Figure 3.3 Metrics calculated for Classification 

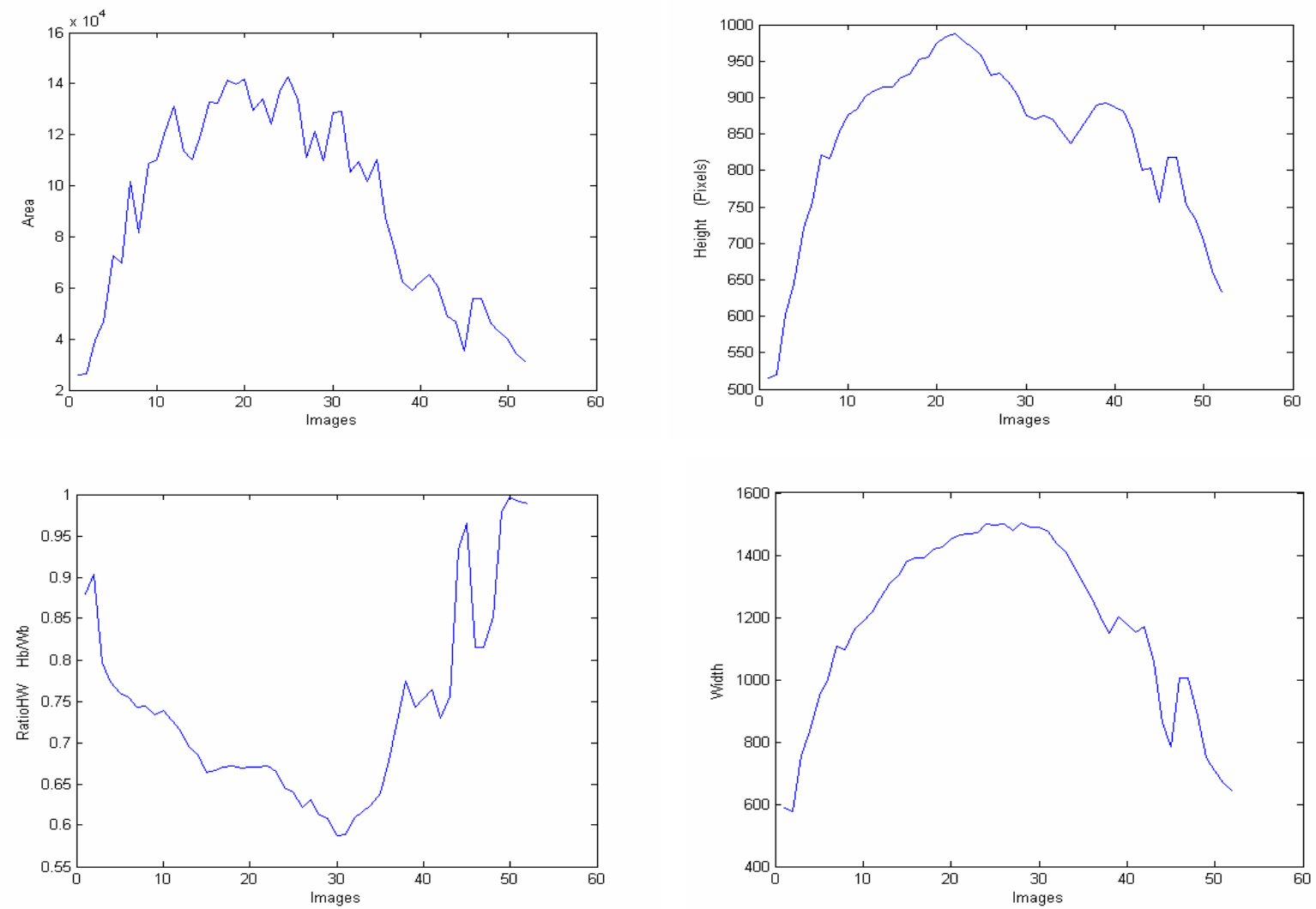

Figure 3.3 Variation of Area, Height, Width, Ratio of Height/Width

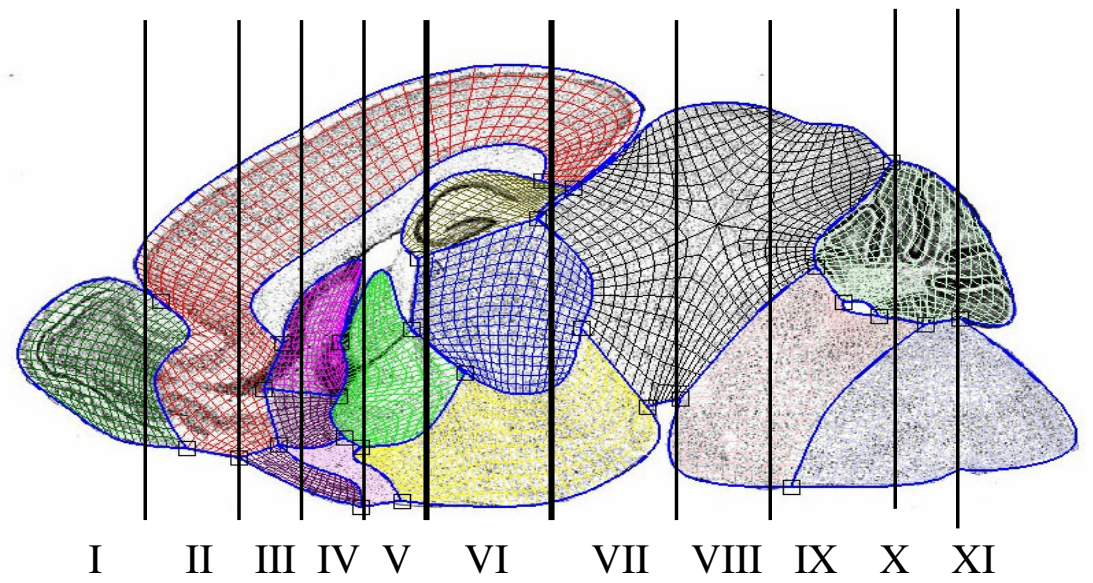

Figure 3.4 Classification of Mouse Brain

The list of image numbers belonging to each class is given in Appendix A. Using thresholds for different parameters such as $R_{H W}, R_{H c}$ etc., tests were conducted to study the performance for image classification. The true classification rate is the number of images classified correctly over the total number of images used in testing. The performance obtained using various parameters are given in Table 3.1. The combination 
of $R_{H W}, R_{H c}$ and $R_{C}$ gave the best performance. But these metrics could not yield good performance since the shape information is not completely explored using this technique. This motivated us to used Fourier descriptors and radii distribution techniques to solve the problem of image classification.

\begin{tabular}{|c|c|}
\hline Technique & True Classification Rate(\%) \\
\hline $\mathrm{R}_{\mathrm{HW}}, \mathrm{R}_{\mathrm{A}}$ & 88.4615 \\
\hline $\mathrm{R}_{\mathrm{HW}}, \mathrm{R}_{\mathrm{A}}, \mathrm{R}_{\mathrm{C}}$ & 80.769 \\
\hline $\mathrm{R}_{\mathrm{HW}}$ & 71.428 \\
\hline $\mathrm{R}_{\mathrm{HW}}$, Ratio Hr, $\mathrm{R}_{\mathrm{C}}$ & 92.307 \\
\hline
\end{tabular}

Table 3.1 Morphometrics-based Classification

\subsection{FOURIER BASED APPROACH}

After boundary extraction, the shape co-ordinates are used to generate a shape signature for the given query image. Shape signature can be defined as a one dimensional function representing a 2-D shape boundary. The shape signature considered in this stage is centroid distance. The centroid distance function is expressed by the distance of the boundary points from the centroid $\left(x_{c}, y_{c}\right)$ of the shape.

$$
r(t)=\left(\left[x(t)-x_{c}\right]^{2}+\left[y(t)-y_{c}\right]^{2}\right)^{\frac{1}{2}}
$$

The centroid distance representation is invariant to translation and rotation due to the subtraction of the centroid, which represents the position of the shape, from boundary, coordinates. The distance set does not vary if the shape is rotated or shifted. Figure 3.4 shows a plot of distances between the boundary points and the centroid of a typical brain section. The arrow indicates the initial position and the boundary is traced in the anticlockwise direction.
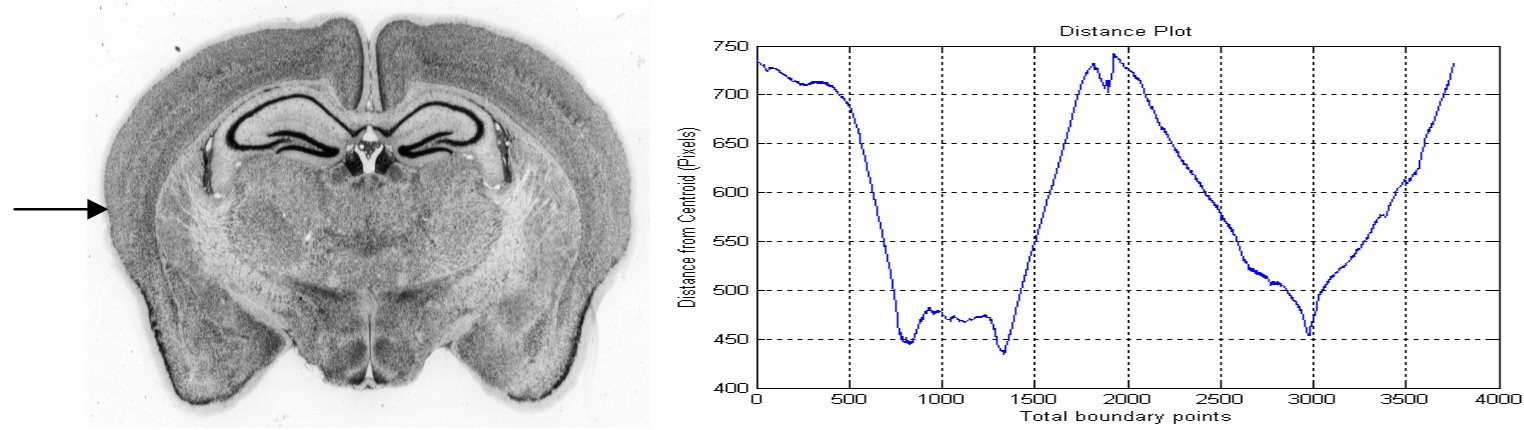

Figure 3.5 Distance between boundary points and centroid 


\subsubsection{Shape Sampling:}

The shape is sampled at a fixed number of points before applying a Fourier transform on the shape signature. The number of data points sampled from the shape signature of objects and models must be the same. To facilitate the use of the Fast Fourier Transform (FFT), the number of sampled points is chosen to be power-of-two integer which we set to 256. The sampling process results in shape normalization. The accuracy of the shape representation depends on the number of sampled points. Sampling can be done in three different ways: equal point sampling, equal angle sampling and equal arc length sampling. After experimenting with the three methods, equal angle sampling gave consistent result with images of different sizes.

Assuming $K$ is the total number of data points to be sampled along the shape boundary, the equal angle sampling technique selects candidate points spaced equal angles $\theta=$ $2 \pi / K$. The equal points sampling method selects the required points spaced at an equal number of points along the shape boundary. The space between two consecutive candidate points is given by $\mathrm{L} / \mathrm{K}$, where $\mathrm{L}$ is the number of total boundary points. In this experiment, we employed equal angle sampling. After sampling, the Fourier transform is applied to the data points.

\subsubsection{D Fourier Transform}

For a two variable function $f(x, y)$ defined in $0 \leq x<N, 0 \leq y<N$, its Fourier transform pair is given by

$$
F(u, v)=\frac{1}{N} \sum_{x=0}^{N-1} \sum_{y=0}^{N-1} f(x, y) \exp [-j 2 \pi(u x+v y) / N]
$$

for $u, v=0,1,2, \ldots \ldots . N-1$

$$
f(x, y)=\frac{1}{N} \sum_{u=0}^{N-1} \sum_{v=0}^{N-1} F(u, v) \exp [j 2 \pi(u x+v y) / N]
$$

for $x, y=0,1,2, \ldots \ldots N-1$

Although the number of $F(u, v)$ resulting from the Fourier transform in the above equation is usually large, the number of significant $F(u, v)$ (or $F(u)$ ) is normally small (significant means $F(u, v)$ with larger magnitude). This is because the higher frequencies 
(less significant coefficients) only represent finest pattern details which may not be important in our problem of shape analysis. This means that a meaningful approximation of original pattern $f(x, y)$ (or $f(x))$ can be constructed from a small number of $F(u, v)$ (or $F(u)$ ). This forms the basis of Fourier based signal processing and Fourier based pattern analysis.

\section{Properties of the Fourier Transform}

The Fourier transform has the following properties, which are useful for pattern recognition.

- Separability: The discrete Fourier transform of (3.2) can be expressed in the separable form

$$
\left.F(u, v)=\frac{1}{N} \sum_{x=0}^{N-1} \exp [-j 2 \pi u x / N] \sum_{y=0}^{N-1} f(x, y) \exp [-j 2 \pi v y) / N\right]
$$

The advantage of the separability is that $F(u, v)$ can be obtained in two steps by successive applications of 1-D Fourier transform which can be computed using the Fast Fourier Transform (FFT).

- Translation: The translation property of the Fourier transform is given by

$$
f\left(x-x_{0}, y-y_{0}\right) \Leftrightarrow F(u, v) \cdot \exp \left[-j 2 \pi\left(u x_{0}+v y_{0}\right) / N\right]
$$

It indicates that a shift in the spatial domain results in phase change in the frequency domain.

- Rotation: If we introduce the polar coordinates with $r$ as radius,

$$
x=r \cos \theta, y=r \sin \theta, u=\omega \cos \varphi, v=\omega \sin \varphi
$$

then substituting the above into the Fourier transform yields

$$
f\left(r, \theta+\theta_{0}\right) \Leftrightarrow F(\omega, \phi+\theta)
$$

In other words, rotating $f(x, y)$ by an angle of $\theta_{0}$ in spatial domain rotates $F(u, v)$ by the same angle in frequency domain.

- Scaling: For two scalars $a$ and $b$, the scaling property of the Fourier transform is given by

$$
f(a x, b y) \Leftrightarrow \frac{1}{a b} F(u / a, v / b)
$$


It indicates the scaling of $f(x, y)$ with $a$ and $b$ in $x$ and $y$ directions respectively in the spatial domain (time domain in 1-D case) causes inverse scaling of the magnitude of $F(u$, $v$ ) in the frequency domain. This implies enlarging an object in the image gives rise to lower frequencies in the spectral domain while shrinking an object in an image gives rise to higher frequencies in the spectral domain.

\section{$\underline{\text { 3.5.3 Discrete Fourier Transform on Shape Signatures: }}$}

The discrete Fourier Transform of shape signature $s(t), t=0.1 \ldots \ldots N-1$, is given by

$$
u_{n}=\frac{1}{N} \sum_{t=0}^{N-1} s(t) \exp \left(\frac{-j 2 \pi n t}{N}\right), n=0,1, \ldots . . N-1
$$

As discussed before, in order to make model shape and data shapes comparable, the shape representations must be invariant to translation, rotation and scale. Rotation invariance of Fourier Descriptors (FDs) is achieved by ignoring the phase information and considering only the magnitude values of the FDs. For centroid distance signature, it is observed that the higher coefficients have less energy and do not produce significant change. Therefore, they are ignored. This implies that one half of the FDs are required to index the shape.

The feature vector can be represented as:

$$
f=\left[\left|F D_{0}\right|,\left|F D_{1}\right|, \ldots \ldots .\left|F D_{N / 2}\right|\right]
$$

Scale invariance is obtained by dividing the magnitude values of the first half of the FDs by the DC component.

$$
f=\frac{\left|F D_{1}\right|}{\left|F D_{0}\right|}, \frac{\left|F D_{2}\right|}{\left|F D_{0}\right|}, \ldots . . \frac{\left|F D_{N / 2}\right|}{\left|F D_{0}\right|}
$$

For a shape in the database indexed by FD feature $f m_{1}=\left[f_{m}{ }^{1}, f_{m}{ }^{2,} \ldots \ldots f_{m}{ }^{N c}\right]$ and the query shape indexed by FD feature $f m_{2}=\left[f_{d}^{l,} f_{d}^{2}, \ldots \ldots F_{d}^{N c}\right]$, the distance measure is computed between the two feature vectors using the Euclidean distance.

$$
d=\left[\sum_{i=0}^{N_{c}}\left|f_{m}{ }^{i}-f_{d}{ }^{i}\right|^{2}\right]^{1 / 2}
$$

The query shape is compared with the ten shapes in the database, where each shape represents the shape of that particular group. 


\subsection{RADIAL DISTRIBUTION BASED APPROACH}

In this technique, the distribution of the centroid distance is used to classify the images. Since all the images are sampled at the same number of points, the number of centroid distances is constant. Using the radial distribution of all the images in the group, a group representative distribution $\left(H_{g}\right)$ is obtained by computing the mean and median distribution. It was observed from the performance of image classification, that the median group representative yields a better result. After the computing the corresponding distribution for the query image, it is compared with the 11 histograms $\left(H_{1}, H_{2} \ldots H_{11}\right)$. The performance is measured using two similarity measures: Kullback-Leibler divergence and simple Euclidean distance. The histogram of the group yielding the least distance is the group to which the given image belongs.

\subsubsection{Centroid-Radii Model:}

The basic idea of this model is to use the lengths of the shape's radii from centroid to boundary, to represent the shape. The distances are computed using the centroid distance function as in Eq. (3.1). The interval between radii, measured in degrees $\theta$, is fixed. The number of intervals can be calculated by $\mathrm{K}=360 / \theta$. The intervals are taken counterclockwise, starting from the $x$-axis. The shape can then be represented by the following vector:

$$
D=\left\{D_{0}, D_{1 \theta}, D_{2 \theta} \ldots \ldots \ldots D_{(k-1) \theta}\right\} \quad \text { where } 0 \leq i \leq(k-1)
$$

In the above equation, $D_{i \theta}$ is the (i+1)th radius from centroid to the boundary of the shape. To make this model invariant to scale, the query image is resized to the size of the database images. This model is invariant to rotation since the centroid and set of distances remains same even if the image is rotated. Figure 3.5 shows different brain sections and a corresponding probability distribution of the radial distances. In the figures below, images from four different classes are used to illustrate the variation in the distribution among different classes. 

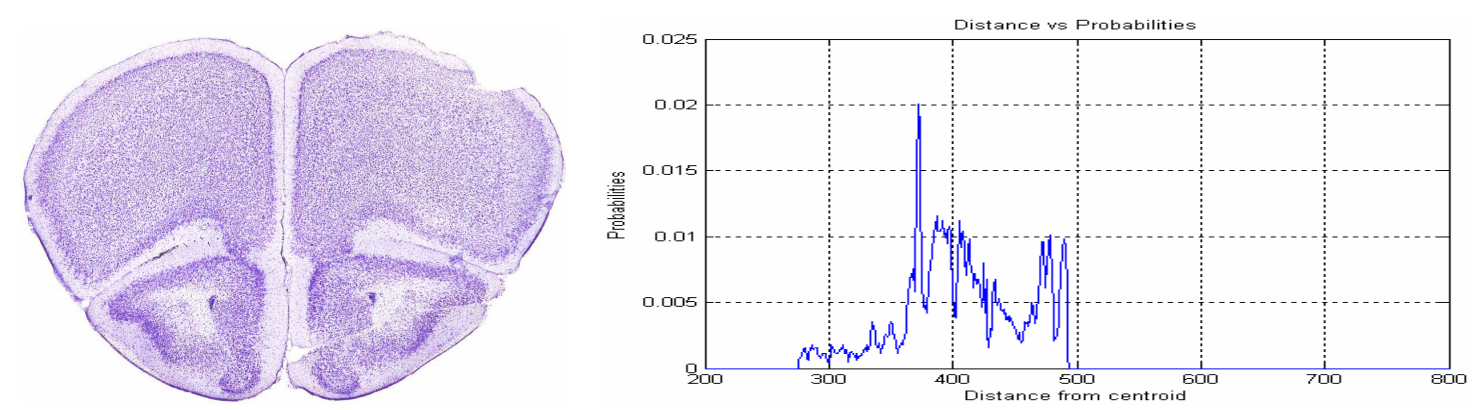

Figure 3.6(a) Class II, Image 11
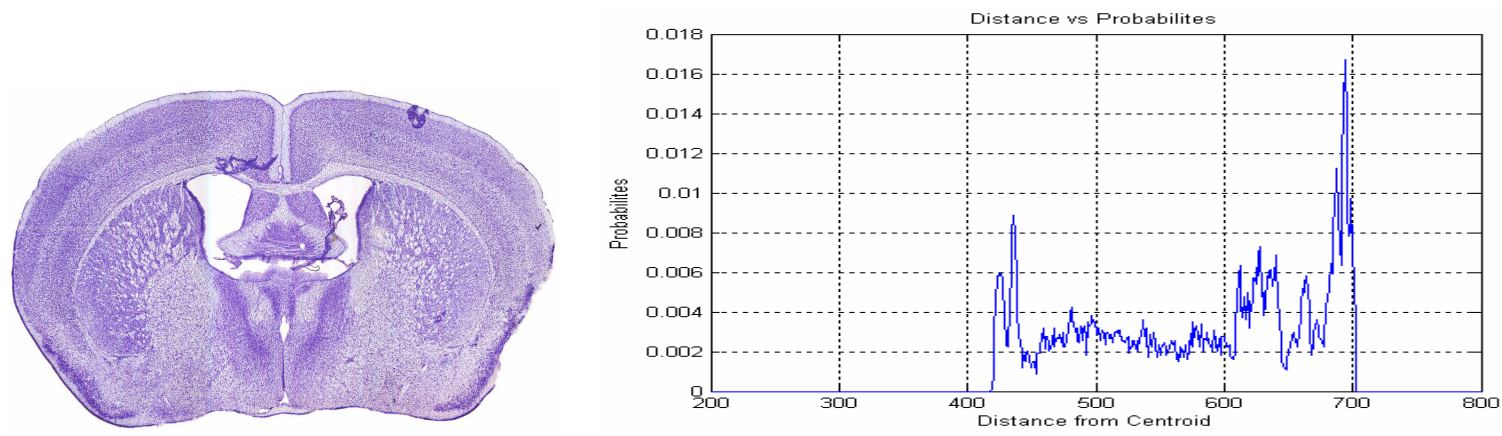

Figure 3.6(b) Class V, Image 33
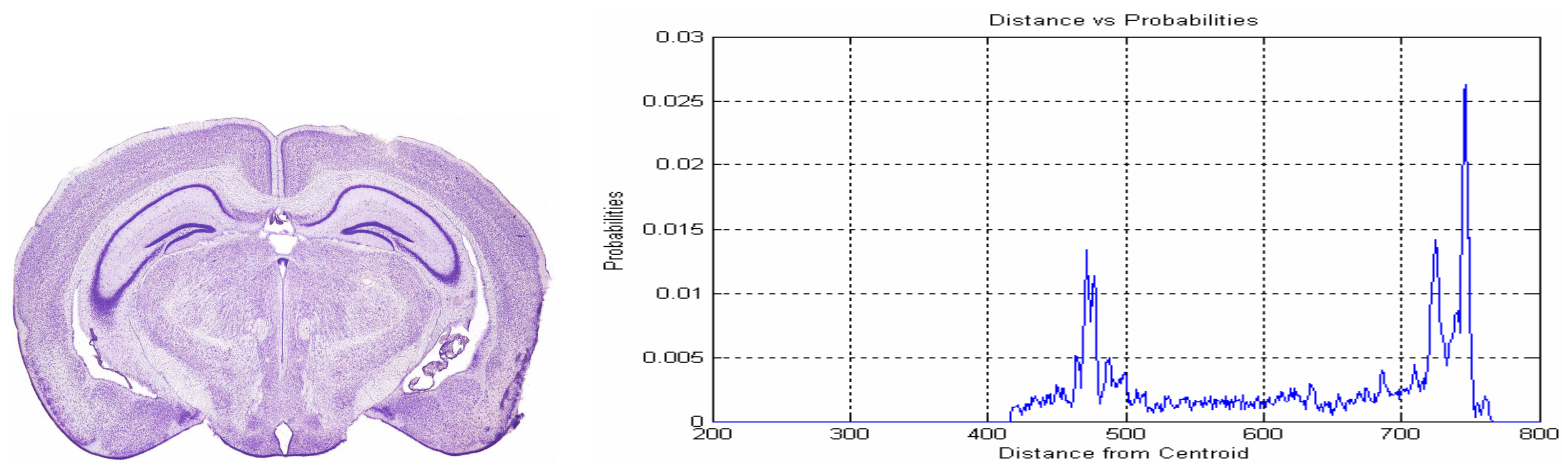

Figure 3.6(c) Class VI, Image 51
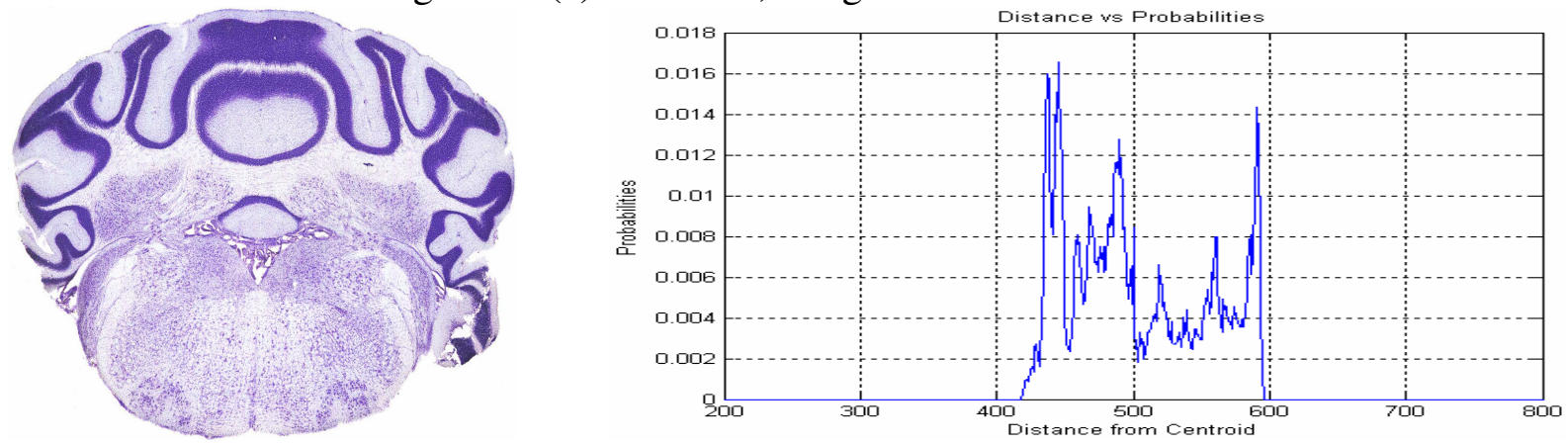

Figure 3.6(d) Class IX, Image 83

To compute the distance between distributions, first the range of all distances $\left[0, D_{\max }\right]$ is divided into $n$ ranges. That is, the ranges of the distance can be represented as $\left[0, D_{\max } / n\right]$, 
$\left[D \max / n, \quad 2 \times D_{\max } / n\right], \quad\left[2 \times D_{\max } / n, \quad 3 \times D_{\max } / n\right], \ldots \ldots,\left[(n-1) \times D_{\max } / n, D_{\max }\right]$. Then, the probability that a distance is within each range is calculated. In our experiment, it is observed that the highest performance was achieved at $n=700$. We calculated the median distribution of all images in a class which is considered as the class representative. Therefore, the radii distribution of the given query image is compared with the 11 template median radii distributions. Table 3.2 shows the performance using different number of bins or ranges, and different group representations (mean distribution and median distribution).

\begin{tabular}{|c|c|c|c|}
\hline Distance Measure & Representative Radial Distribution & Range & True Classification Rate(\%) \\
\hline KL-Distance & Mean & 500 & 81.13 \\
\hline KL-Distance & Mean & 600 & 94.34 \\
\hline KL-Distance & Mean & 700 & 94.34 \\
\hline KL-Distance & Mean & 800 & 90.57 \\
\hline KL-Distance & Mean & 900 & 88.67 \\
\hline KL-Distance & Mean & 1000 & 88.67 \\
\hline KL-Distance & Mean & 1100 & 88.67 \\
\hline KL-Distance & Median & 500 & 81.13 \\
\hline KL-Distance & Median & 600 & 90.57 \\
\hline KL-Distance & Median & 700 & 95.8 \\
\hline KL-Distance & Median & 800 & 86.79 \\
\hline KL-Distance & Median & 900 & 86.79 \\
\hline KL-Distance & Median & 1000 & 86.79 \\
\hline
\end{tabular}

Table 3.2 Effect of bin size on performance

Let $\mathrm{H}^{\mathrm{DI}}$ represent the radial distribution of the template images in class I, the radial distribution of each image can be represented as:

$$
\begin{aligned}
& H_{1}^{D I}=\left[h_{11}, h_{21}, \ldots \ldots \ldots . . . h_{L 1}\right] \\
& H_{2}^{D I}=\left[h_{12}, h_{22}, \ldots \ldots \ldots . . . h_{L 2}\right] \\
& \cdot \\
& H_{c}^{D I}=\left[h_{1 c}, h_{2 c}, \ldots \ldots \ldots . . . h_{L c}\right]
\end{aligned}
$$

Where, $c$ is the number of images in the class I and $L$ is the number of bins. The mean radii distribution $\mathrm{H}^{\mathrm{M}}$ is given by: 


$$
H^{M I}=\left[h_{1}, h_{2}, \ldots . . h_{L}\right], \quad \text { where } h_{i}=\frac{1}{c} \sum_{k=1}^{c} h_{k}
$$

The median radial distribution $\mathrm{H}^{\mathrm{MD}}$ is given by:

$$
H^{M D I}=\left[\hat{h_{1}}, \hat{h}_{2}, \ldots \ldots . \hat{h_{L}}\right], \text { where } \hat{h}_{i}=\underset{k}{\operatorname{median}}\left\{h_{k i}\right\}
$$

The variation in the performance of image classification with range is illustrated using Figure 3.6. This figure shows the performance using both KL and Euclidean distances.

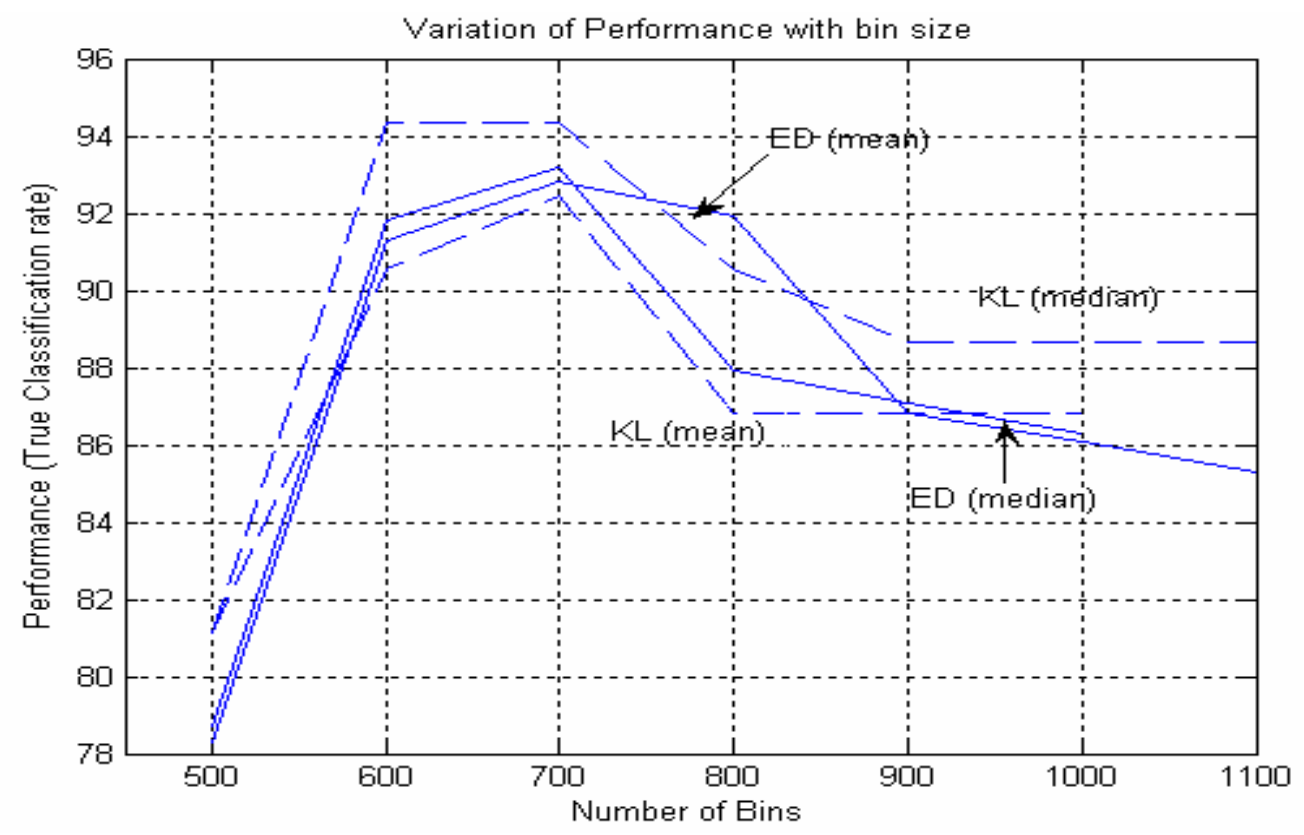

Figure 3.7 Variation in Performance with bin size

\section{$\underline{\text { 3.6.2 Similarity Measurement: }}$}

The radial distribution can be represented as:

$$
D=\left(d_{0}, d_{1}, d_{2}, \ldots . . d_{(n-1)}\right)
$$

where, $\mathrm{n}$ is the number of ranges used in the representation and $d_{i}, i \in[0, n-1]$ is the number of radial distances within this range. To compare two shapes with radial distributions as given by $D_{1}$ and $D_{2}$ :

$$
D_{1}=\left(d_{10}, d_{11}, d_{12}, \ldots . . d_{1(n-1)}\right)
$$

and

$$
D_{2}=\left(d_{20}, d_{21}, d_{22}, \ldots . . d_{2(n-1)}\right)
$$

We again calculate Euclidean distance between the two distributions. 


$$
S\left(D_{1}, D_{2}\right)=\sqrt{\sum_{i=0}^{n-1}\left(d_{1 i}-d_{2 i}\right)^{2}}
$$

Since we are dealing with probabilities, we also used the Kullback-Leibler divergence to measure the similarity between the radial distributions. After testing the algorithm, it has been observed that this similarity measure gives better performance compared to the Euclidean distance measure. The symmetric form of KL-distance is given by:

$$
S_{K L}\left(d_{1}, d_{2}\right)=\frac{1}{2}\left[\sum_{i=0}^{n-1} P\left(d_{1 i}\right) \log \left(\frac{P\left(d_{1 i}\right)}{P\left(d_{2 i}\right)}\right)+\sum_{i=0}^{n-1} P\left(d_{2 i}\right) \log \left(\frac{P\left(d_{2 i}\right)}{P\left(d_{1 i}\right)}\right)\right]
$$

where $P\left(d_{i}\right)$ is the normalized form such that $\sum d_{1 i}=1$, and $\sum d_{2 i}=1$

After image classification, the next stage of the automated image mapping system is to perform an image matching algorithm to determine the exact location of the given image on the mouse brain axis. In the following, we focus on only images within Group VI for the stage of image mapping. 


\section{IMAGE MATCHING}

\subsection{INTRODUCTION}

After classification into a group, the exact location of the query image within the group requires more fine-grained analysis, beyond the capability provided by boundary-based shape representations. The two stages in the automated image mapping system are as shown in the figure 4.1. Thus, we considered two approaches to identify the best match: morphometrics-based approach and a region-based approach, using the texture information as our basic feature. Texture is playing an increasingly important role in computer vision. It has found wide applications in remote sensing, medical diagnosis, quality control, biometrics etc.

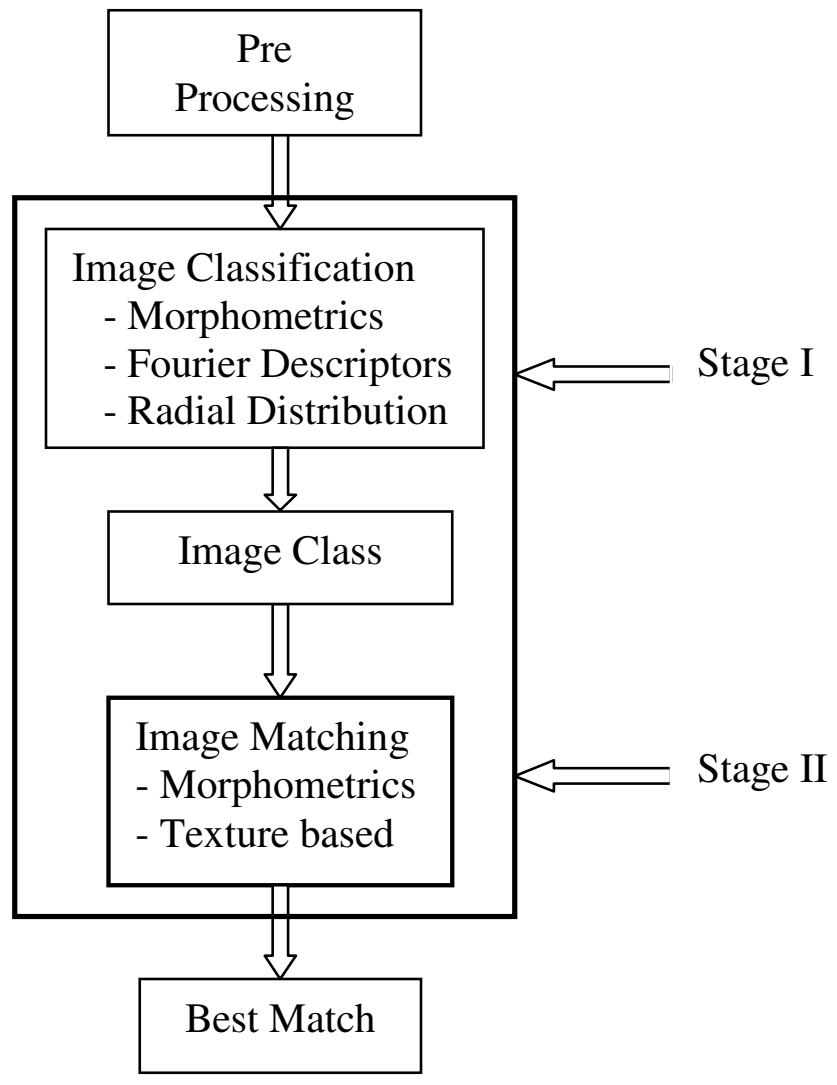

Figure 4.1 Block Diagram of Dual Stage Approach

In this thesis, the second stage of AIMS, Image Matching, uses texture information in the mouse brain images to obtain similar images from the database. When the image is identified to be in the Group VI, hippocampal region of the brain, the texture of the hippocampus region in the image is used to retrieve the most relevant image in the 
database. The hippocampus is a structure of the limbic system in the brain that integrates incoming nerve impulses to the hypothalamus, and is also the memory center for the brain. The length of the hippocampus varies along the brain axis as shown in Class VI of Appendix A. The curved elevation of gray matter extending the entire length of the floor of the temporal horn of the lateral ventricle is used to obtain the best match.

\subsection{MORPHOMETRIC BASED MATCHING}

Among the class VI images, it can be seen that size of the hippocampus can be used as a feature to determine the exact match. We used a landmark-based method to perform matching. User input is required to detect the landmarks. After identifying the positions, as shown in the Figure 4.2, various measurements are calculated which help in determining the exact position of the given query image on the brain axis. These measurements include $L_{H}$ (Length of Hippocampus), $L_{C}$ (Length of the image), $R_{H}\left(L_{H} /\right.$ $L_{C}$ ) where $C$ represents centroid and $\theta_{\mathrm{H}}$. The variation of these features along the brain axis is illustrated using Figure 4.3. A simple Euclidean distance measure gives the distance of the given query from images in the database. We can observe that the morphological features can predict the position of the section along the brain axis, once the group has been determined. However, though this technique gives a very high performance, the main drawback is that it requires user input. In order to make this system automated, we employed a texture-based matching technique for the second stage of the Image Matching system. This approach is discussed in detail in the following sections.

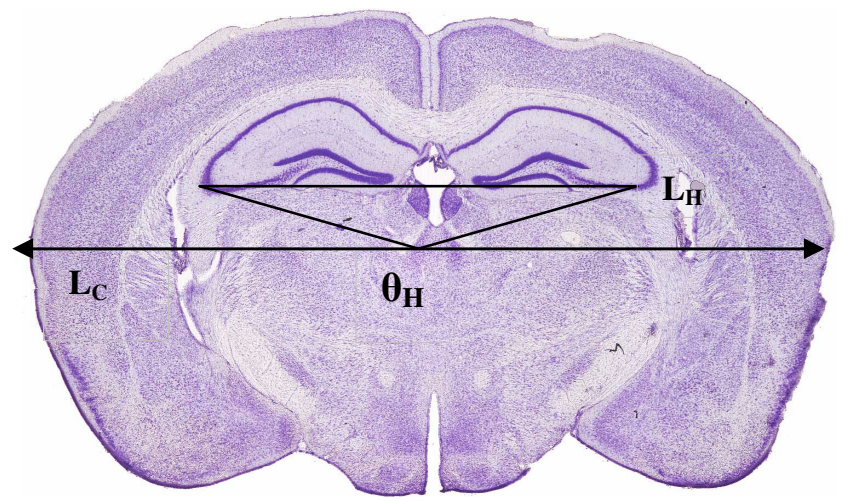

Figure 4.2 Landmark Detection 

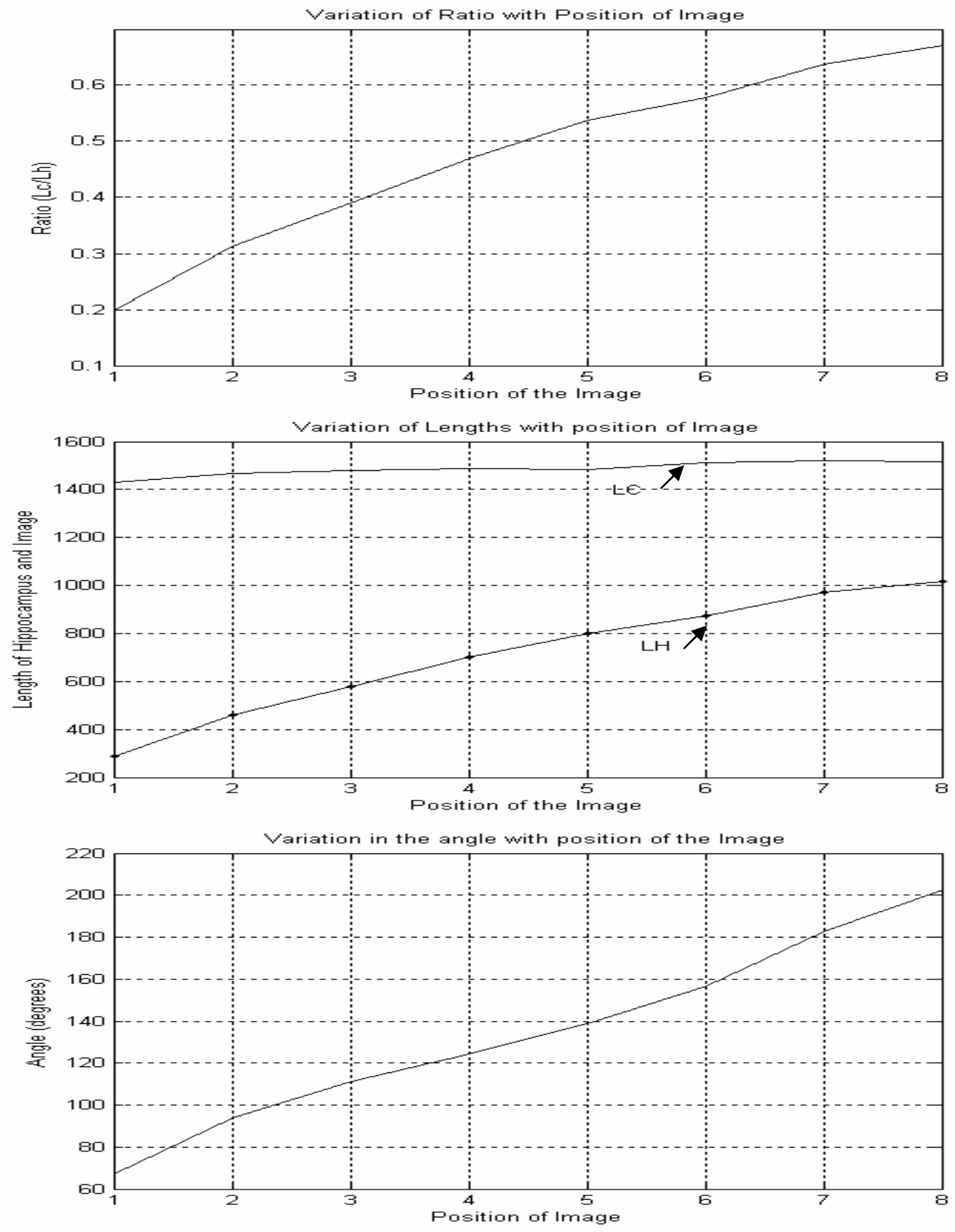

Figure 4.3 Variation of features with position of the Image

\subsection{TEXTURE BASED MATCHING}

The second method is texture based matching which consists of two parts: region selection and template matching. In the given image, the region of the hippocampus is collected to extract features using Gabor filters. This region is divided into blocks which have different texture and whose boundaries coincide with spatial edge locations. Each block or valid combinations of blocks constitute "potential objects". In the second part, 
the texture of each object is compared with the features extracted from the template. This technique is translation, rotation and scale invariant which is due to image resizing where the features of the query object and template object are extracted using Gabor filters.

The motivation for using Gabor filters in extracting texture image features is that they have been shown to be optimal in the sense of minimizing the joint two-dimensional uncertainty in space and frequency [61]. Gabor filters are a collection of wavelets, where each wavelet can capture the energy of one particular spatial frequency and in one specific orientation.

\subsubsection{Gabor Wavelets}

For a given image $I(x, y)$ of size $N \times N$, the discrete Gabor wavelet transform is given by $[61]$,

$$
E_{m n}(x, y)=\sum_{k} \sum_{l} I(x, y) g_{m n}^{*}(x-k, y-l)
$$

where, $k, l$ are the filter mask variables and $m, n$ are scale and orientation parameters. The function $g^{* m n}$ is the complex conjugate of a two dimensional Gabor function. The Gabor functions are obtained for a specific $m$ and $n$ pair. In general a two dimensional Gabor function can be written as,

$$
g(x, y)=\frac{1}{2 \pi \sigma_{x} \sigma_{y}} \exp \left[-\frac{1}{2}\left(\frac{x^{2}}{\sigma_{x}^{2}}+\frac{y^{2}}{\sigma_{y}^{2}}\right)+j 2 \pi F x\right]
$$

where, $F$ is the modulation frequency. A class of self-similar functions called Gabor wavelets, are obtained by appropriate dilations and rotations of $g(x, y)$ using the generating function,

$$
g_{m n}(x, y)=a^{-m} g\left(x^{\prime}, y^{\prime}\right) \quad a>1
$$

where, $m$ and $n$ specify the scale and orientation of the wavelet respectively, with $m=0$, $1, \ldots, \Psi-1, n=0,1, \ldots, \Omega-1, \Psi=$ number of scales and $\Omega=$ number of orientations in the multi-resolution decomposition. And

$$
x^{\prime}=a^{-m}(x \cos \theta+y \sin \theta)
$$




$$
\begin{aligned}
& y^{\prime}=a^{-m}(-x \sin \theta+y \cos \theta) \\
& \theta=n \pi / \Omega
\end{aligned}
$$

The variables in the above equations are defined as:

$$
\begin{aligned}
& a=\left(\frac{U_{h}}{U_{l}}\right)^{\frac{1}{S-1}}, \\
& F=U_{h} \\
& \sigma_{x_{m n}}=\frac{(a-1) U_{h}}{(a+1) \sqrt{2 \ln 2}} \\
& \sigma_{y_{m n}}=\frac{\tan \left(\frac{\pi}{2 \Omega}\right)\left[U_{h}-2 \ln \left(\frac{\sigma_{x_{m n}}}{U_{h}}\right)\right]}{\sqrt{\left[2 \ln 2-\frac{(2 \ln 2)^{2} \sigma_{x_{m n}}^{2}}{U_{h}}\right]}}
\end{aligned}
$$

$U_{h}$ and $U_{l}$, are the upper and lower center frequencies respectively. In this work, we use $U_{h}=0.4, U_{l}=0.05$ and $\Psi=4, \Omega=6$ to generate the Gabor wavelets. Based on the assumption that a Gabor wavelet transformed texture image is spatially homogenous, Manjunath and Ma [61] used the mean $\mu_{m n}$ and standard deviation $\sigma_{m n}$ of the magnitude of the transformed coefficients to represent the image for classification and retrieval. In this work, the mean and standard deviation of the magnitude of the Gabor transform coefficients are used to extract features.

After dividing the hippocampus region into the required blocks, the texture information in the blocks is transformed using Gabor wavelets as explained using equations above. The mean and standard deviation are computed using the equations given below.

$$
\begin{aligned}
\mu_{m n} & =\frac{1}{N^{2}} \sum_{x=0}^{N-1} \sum_{y=0}^{N-1}\left|E_{m n}(x, y)\right| \\
\sigma_{m n} & =\sqrt{\frac{1}{N^{2}} \sum_{x=0}^{N-1} \sum_{y=0}^{N-1}\left|E_{m n}(x, y)-\mu_{m n}\right|}
\end{aligned}
$$




\subsubsection{Region based template matching}

The blocks in the region of interest are collected as shown in the Figure 4.3. The same procedure is repeated for the images in the database. A representation of the blocks on the query and database image is shown in Fig 4.3. The motivation in using the region-based matching technique is illustrated using Figure 4.4. Observe that the position of the blocks with the same texture is varying with the position of the image on the brain axis. This observation is summarized in the schematic diagram in Figure 4.5. Using the information of this position, and extracting texture features, solves the problem of identifying the best match for the given image in the hippocampus region. After obtaining the features from these blocks using Gabor wavelets, they are compared with the corresponding blocks in all the images in the group.

Normalizing the feature values and using suitable distance measure can improve discrimination ability of the features. Below we describe a method to determine a normalization vector of the feature values.
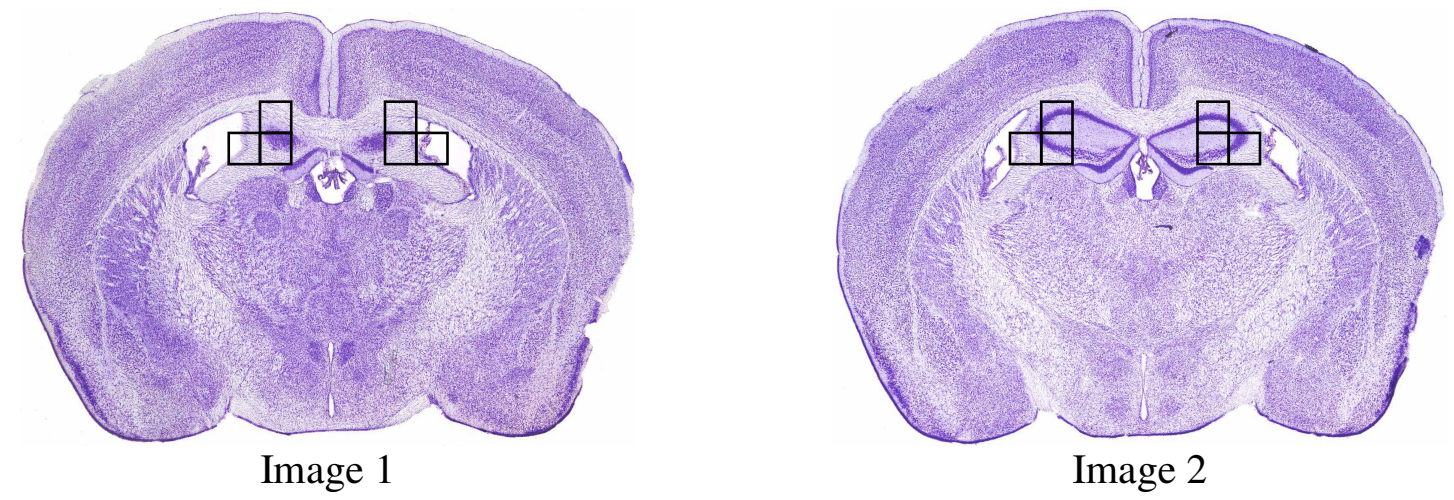

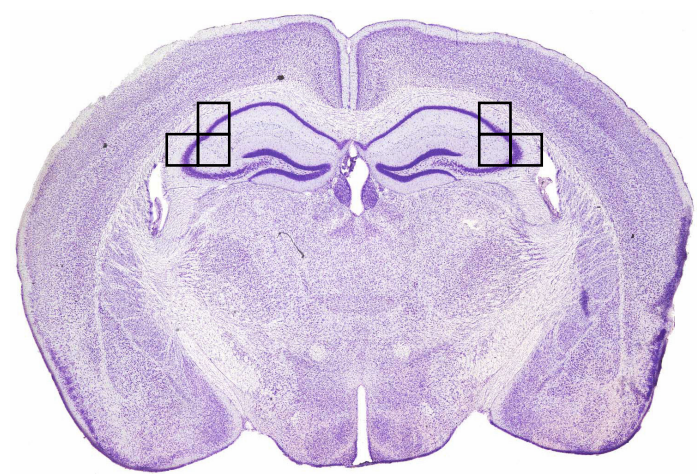

Image 3

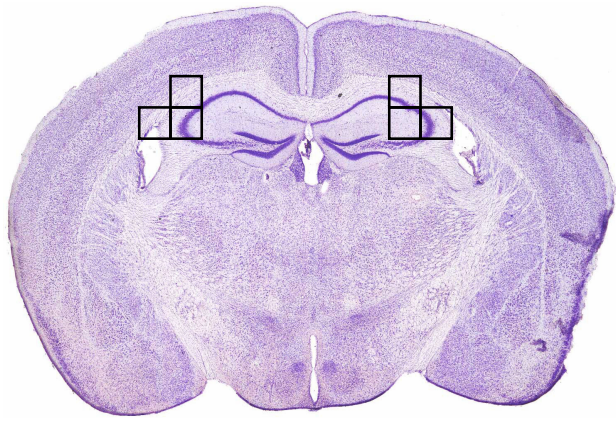

Image 4 

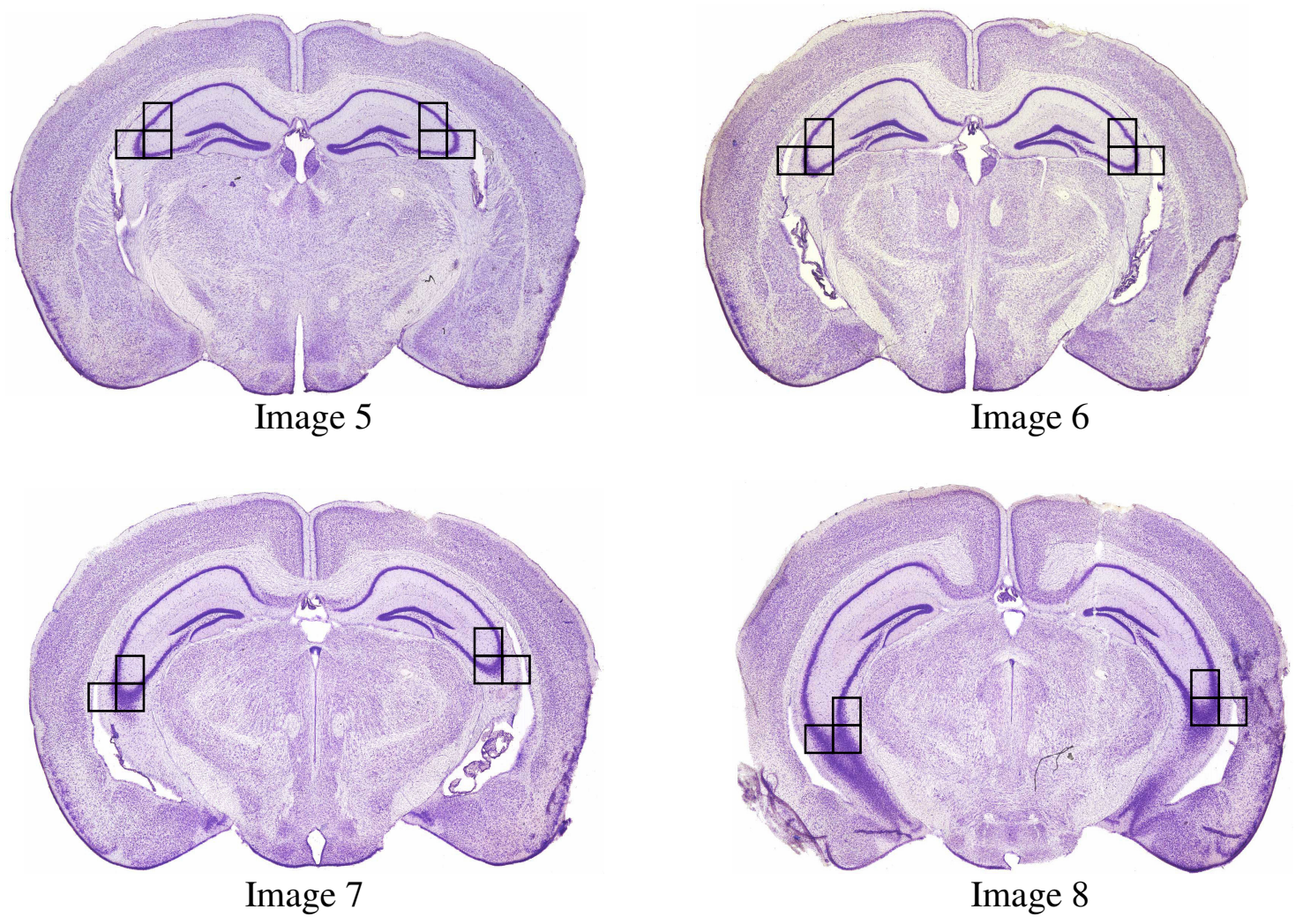

Figure 4.4: Distinguishing Feature blocks in each image in the hippocampus group
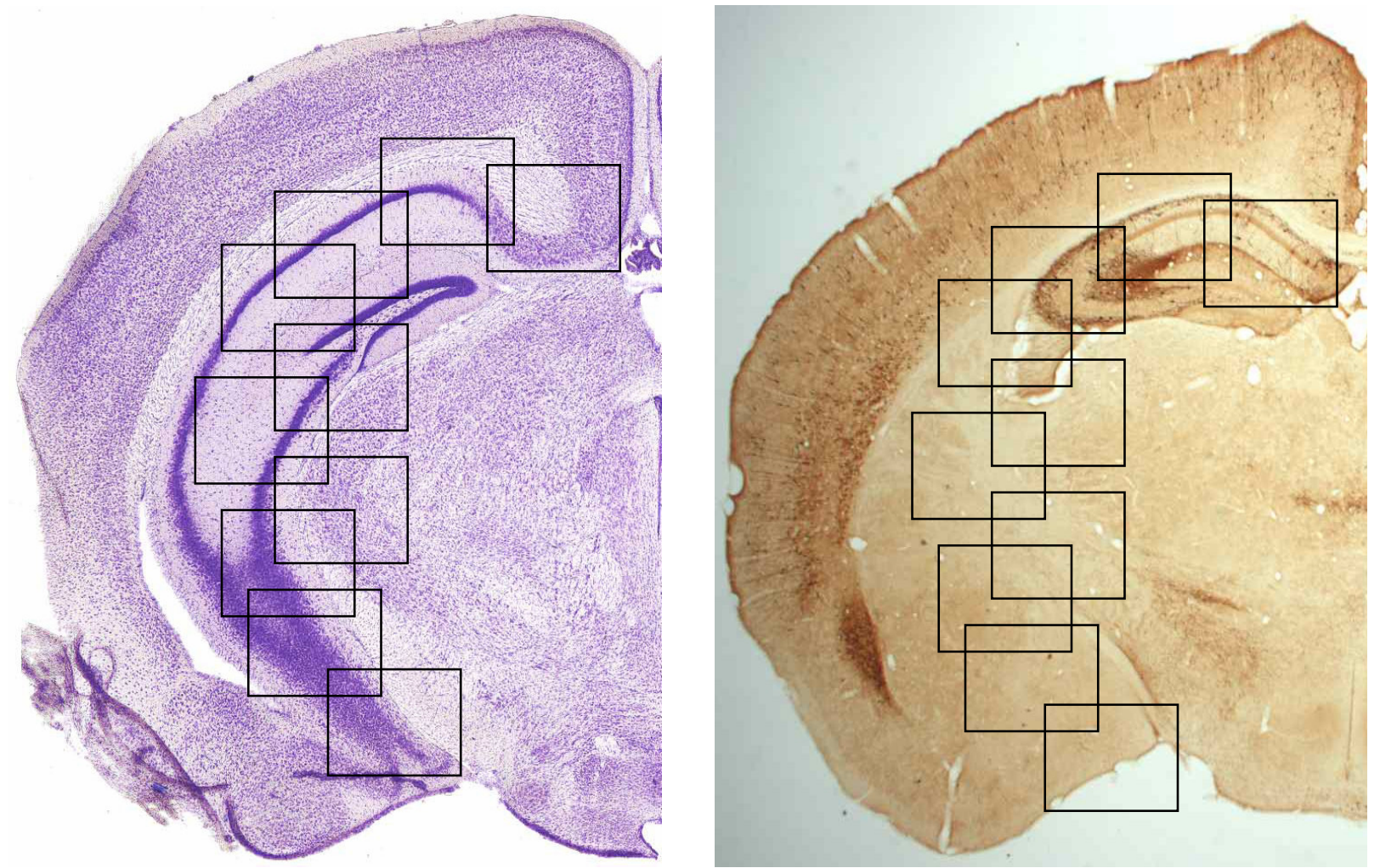

Figure 4.5: Template image and test image with regions of interest 


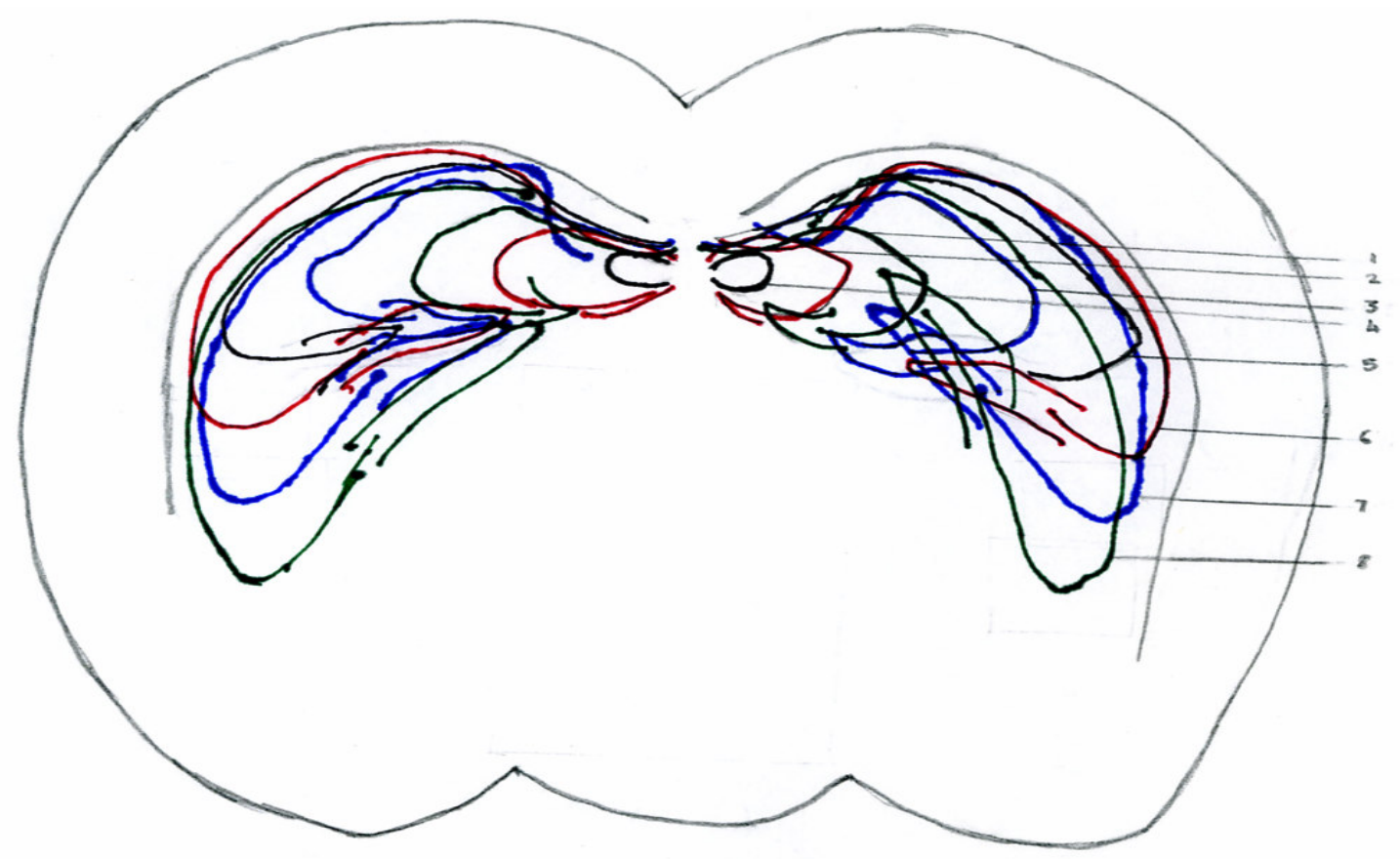

Figure 4.6: Evolution of the Hippocampus size withing Group VI

The feature vector $f_{i}$ of block $i$ is a collection of features $\left\{f_{m n}\right\}$ that are extracted from the corresponding transformed information $\left\{E_{m n}\right\}$.

$$
\begin{aligned}
& f^{(i)}=\left[\begin{array}{llllll}
f_{11}^{(i)} & f_{12}^{(i)} & \cdot & \cdot & f_{m n}^{(i)}
\end{array}\right]^{T} \\
& =\left[\begin{array}{llllll}
s\left\{E_{11}\right\} & s\left\{E_{12}\right\} & \cdot & \cdot & s\left\{E_{m n}\right\}
\end{array}\right]^{T}
\end{aligned}
$$

where, $s$ is the feature extraction function and $m, n$ are the number of scales and orientations respectively. The product of $m$ and $n$ is the total number of channels that are being used for multi-channel image analysis. The image database contains the feature matrix $F$ :

$$
F=\left\{f^{(1)} f^{(2)} \cdot \cdot \cdot f^{(i)} \cdot \cdot \cdot f^{(N)}\right\}
$$

where, $N$ is the total number of images present in the database. To exploit the structural information available in each channel, the features values in each channel are first normalized with respect to the maximum feature value available in that particular channel. This implies, each row in the feature matrix is normalized with respect to the highest value available in that particular row. 
The normalized feature matrix $\hat{F}$ can be represented as

$$
\hat{F}=\left\{\begin{array}{llllll}
\hat{f}^{(1)} & \left.\hat{f}^{(2)} \cdot \hat{f}^{(i)} \cdot . \cdot \hat{f}^{(N)}\right\}
\end{array}\right.
$$

where

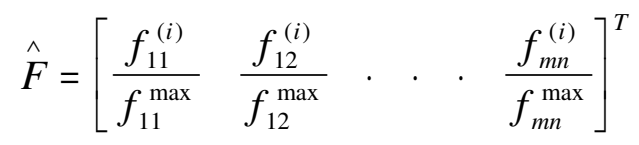

where, $\quad f_{i j}^{\max }=\max _{i j}\left\{f_{i j}^{(1)} f_{i j}^{(2)} \cdot \cdot f_{i j}^{(N)}\right\}$

After normalization, the normalized city-block distance is used to compare the texture features. Let $i, j$ be the two texture blocks, the distance between the patterns is given as:

$$
d(i, j)=\sum_{m} \sum_{n} d_{m n}(i, j)
$$

Where,

$$
d_{m n}(i, j)=\left|\frac{\mu_{m n}^{(i)}-\mu_{m n}^{(j)}}{\alpha\left(\mu_{m n}\right)}\right|+\left|\frac{\sigma_{m n}^{(i)}-\sigma_{m n}^{(j)}}{\alpha\left(\sigma_{m n}\right)}\right|
$$

Where $\alpha\left(\mu_{m n}\right)$ and $\alpha\left(\sigma_{m n}\right)$ are the standard deviation of the respective features over the entire corresponding database sets. These serve as normalizing constants for each individual feature components. Testing this technique with a large query set, it is observed that the performance is high for histological images and computational cost is low. 


\section{EXPERIMENTS AND PERFORMANCE ANALYSIS}

\subsection{EXPERIMENTAL DATA}

To test the performance of the proposed approach on histological images, 14 mouse brains were used. The coronal sections were obtained by cryosectioning at $20 \mu \mathrm{m}$ thickness. The sections were stained with cresyl violet or nissl stain. The reference set, the Paxinos and Watson atlas [16] consists of 100 evenly spaced sections extending across the brain length from the level of the anterior olfactory nucleus to the caudal cerebellum. For the test set, 341 sections from14 different mouse brains of about 25 sections per mouse, were randomly selected. Table 5.1 shows the list of data sets used in the experiments. The test images are acquired from Dr. Agmon's Neuroanatomy Laboratory (NBAN) at Health Sciences Center, in West Virginia University and Mouse Brain Library, University of Tennessee [27]. The test section is fed to the automated image matching system and the computer displays the best match for a given query.

\begin{tabular}{|c|c|c|c|c|c|}
\hline Source & Strain & Cutting Plane & Sections & \# of Mice & Group VI Images \\
\hline NBAN Lab & P7 C57BL/6 & Coronal & 66 & 4 & 27 \\
\hline MBL & C57BL/6J & Coronal & 90 & 3 & 25 \\
\hline MBL & DBA/2J & Coronal & 60 & 2 & 19 \\
\hline MBL & BALB/cJ & Coronal & 25 & 1 & 7 \\
\hline MBL & A/J & Coronal & 25 & 1 & 7 \\
\hline MBL & NOD/LtJ & Coronal & 25 & 1 & 6 \\
\hline MBL & C57L/J & Coronal & 25 & 1 & 7 \\
\hline MBL & $129 X 1 / S v J$ & Coronal & 25 & 1 & 7 \\
\hline Total & & $\mathbf{3 4 1}$ & $\mathbf{1 4}$ & $\mathbf{1 0 5}$ \\
\hline
\end{tabular}

Table 5.1 Summary of Experimental Data

\subsection{EXPERIMENTAL ENVIRONMENT}

The experiments were carried out in a MATLAB 7.1 environment, using DELL personal computers with Pentium M processor running at $1.8 \mathrm{GHz}$ with $1 \mathrm{~GB}$ RAM.

\subsection{PERFORMANCE MEASURES}

Performance was measured by comparing results of automatic classification with those from matching by a human. The automatic classification relied only on the external contour whereas the expert identified the homologous section pairs using the gray scale 
images and could employ both internal and external features. Due to variation in cutting angle between the reference and test sets, for some test sections the expert could identify only an imperfect match which is used for comparison with computer performance. Results are calculated by evaluating the number of sections separating the computer and human selected atlas match for a given test section.

In Fig 5.1, the machine results using the proposed method are compared to those of the human. The performance was calculated from the results of the query set which consists of 341 test sections. In this experiment, individual test sections were matched to homologous reference sections.

\subsection{RESULTS FOR IMAGE CLASSIFICATION}

Performance is measured based on the number of images correctly classified. The precision can be defined as the degree of discrimination. If $Q$ is the number of images correctly classified in the groups, and $N$ is the total number of query images used to test the system, precision is evaluated as

$$
P=Q / N
$$

The Table 5.2 shows the results obtained using stage I of the automated image matching system. The query set of 341 images is classified into eleven groups. For Fourier Descriptors, the experiments were conducted with 64 data points with equal angle between them. The radial distribution technique has been tested with different ranges varying from 500-1000. The optimum performance was achieved with 700 number of bins. Results show average precision with three different techniques. Since the extracted contours used in this classification method are sometimes noisy the success rate drops down to a nominal $87.39 \%$. On the other hand, classification based on radial distribution gave $95.9 \%$ of average success. Another advantage with this technique is that the process time is less compared to all other methods. The performance of KL-distance is better compared to that of Euclidean distance when applied to radial distributions. The best result obtained using morphometrics is also shown in the table. 


\begin{tabular}{|c|c|c|c|c|c|}
\hline Technique & $\begin{array}{c}\text { Precision } \\
(\mathbf{\%})\end{array}$ & $\begin{array}{c}\text { \# Input } \\
\text { Images }\end{array}$ & $\begin{array}{c}\text { True } \\
\text { Classification }\end{array}$ & $\begin{array}{c}\text { False } \\
\text { Classification }\end{array}$ & $\begin{array}{c}\text { Elapsed } \\
\text { Time (sec) }\end{array}$ \\
\hline Fourier Descriptors & 87.39 & 341 & 298 & 43 & 5.14 \\
\hline Radial Distribution(KL Mean) & 94.42 & 341 & 322 & 19 & 2.92 \\
\hline Radial Distribution(KL Median) & 95.9 & 341 & 327 & 14 & 2.92 \\
\hline Radial Distribution(ED Mean) & 93.25 & 341 & 318 & 23 & 2.92 \\
\hline Radial Distribution (ED Median) & 93.84 & 341 & 320 & 21 & 2.9 \\
\hline Spline-based & 71.2 & 341 & 243 & 98 & 79 \\
\hline
\end{tabular}

Table 5.2 Comparison of Success Rates of Different Methods

\subsection{RESULTS FOR IMAGE MATCHING OF HIPPOCAMPUS IMAGES}

The performance of stage II is evaluated as a stand-alone system which implies, the input images belong to the Group VI (Hippocampus Images) and is picked by the user testing the system. Despite the fact that texture based matching is considered one of the effective methods for image matching, the success rate in our case of histological image matching is restricted to $76.8 \%$ due to the intensity variations in the histological brain images. The texture information was difficult to obtain in some images which are taken under poor illumination conditions. The success rate at different ranks is tabulated below.

\begin{tabular}{|c|c|}
\hline Ranks & Success Rate(\%) \\
\hline Top 1 & $76.8 \%$ \\
\hline Among Top 2 & $79.6 \%$ \\
\hline Among Top 3 & $95 \%$ \\
\hline Among Top 4 & $100 \%$ \\
\hline Among Top 5 & $100 \%$ \\
\hline
\end{tabular}

Table 5.3 Success Rates of Ranked Matches

\subsection{RESULTS OF DUAL STAGE IMAGE MATCHING SYSTEM}

From the Table 5.2, it is shown that the radial distribution technique gives better performance for the image classification problem. Hence, in the dual-stage matching system, the Stage I is implemented using distribution of radial distances and stage II is implemented using region-based template matching system. The overall performance of the system is observed to be $76.8 \%$ with a total processing time of 11 seconds. Figure 5.2 
gives a summary performance of the mapping scheme for histological sections. The distance mentioned is the error between the machine selected and expert selected images in terms of number of sections separating the two images. For class VI, the maximum distance can be 7. Figure 5.1 shows the distribution of error.

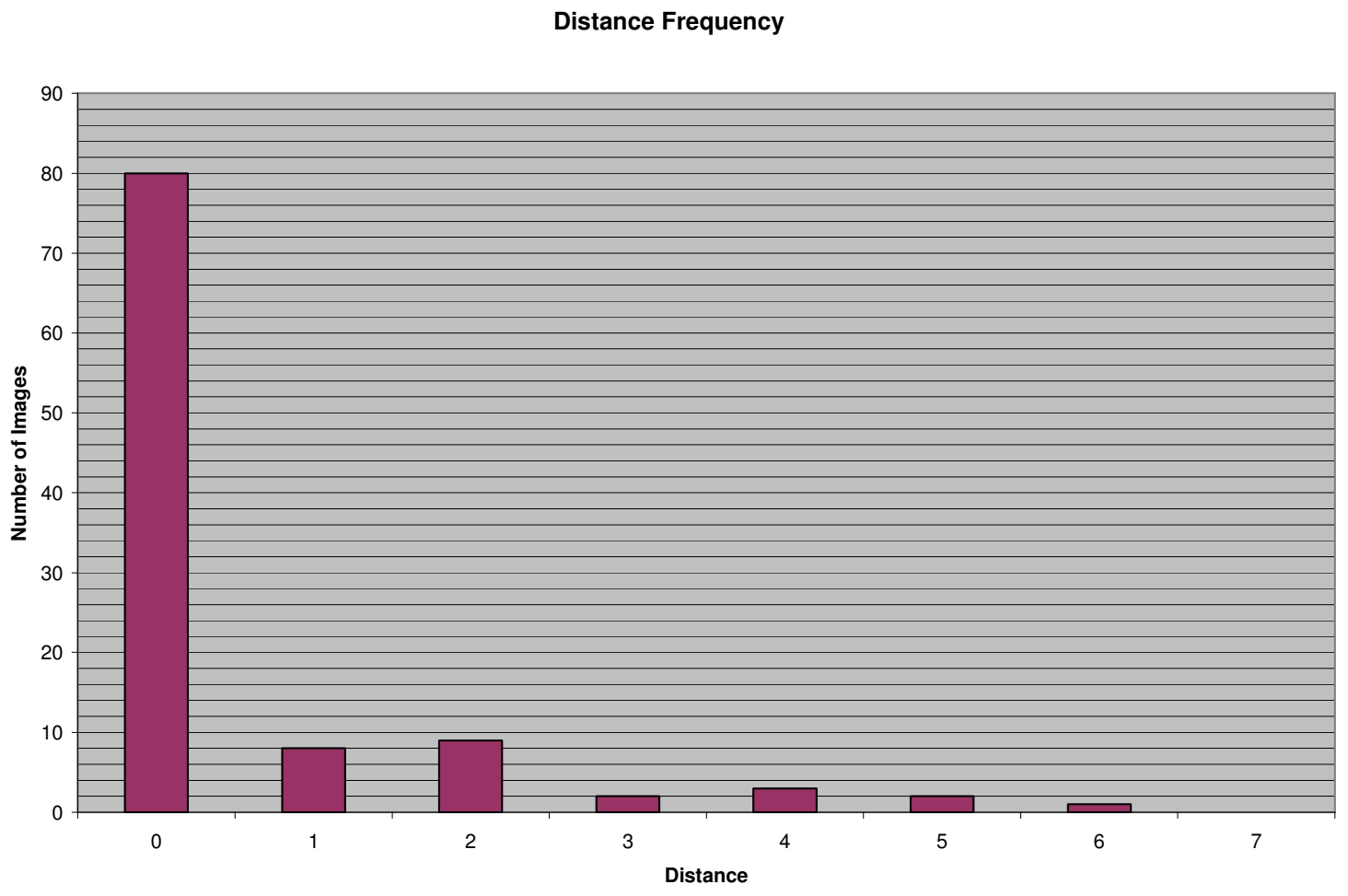

Figure 5.1: Distance Frequency

\section{Query Section}
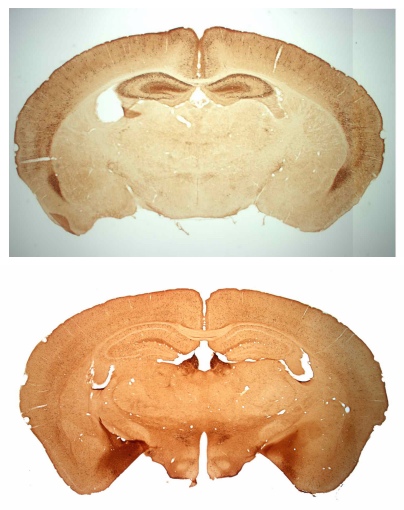

Machine Selected
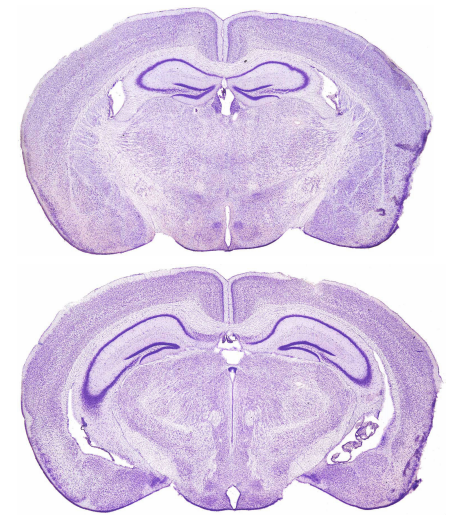

Expert Selected
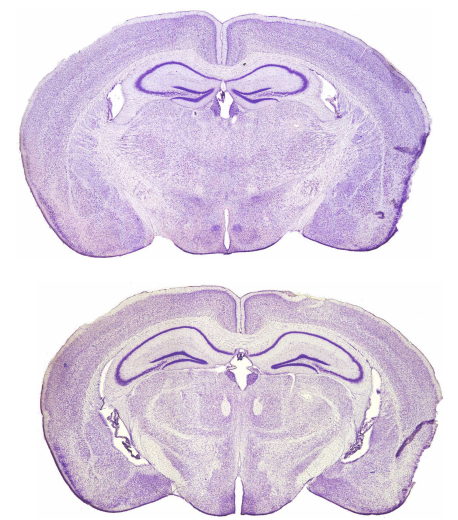

Distance

0 

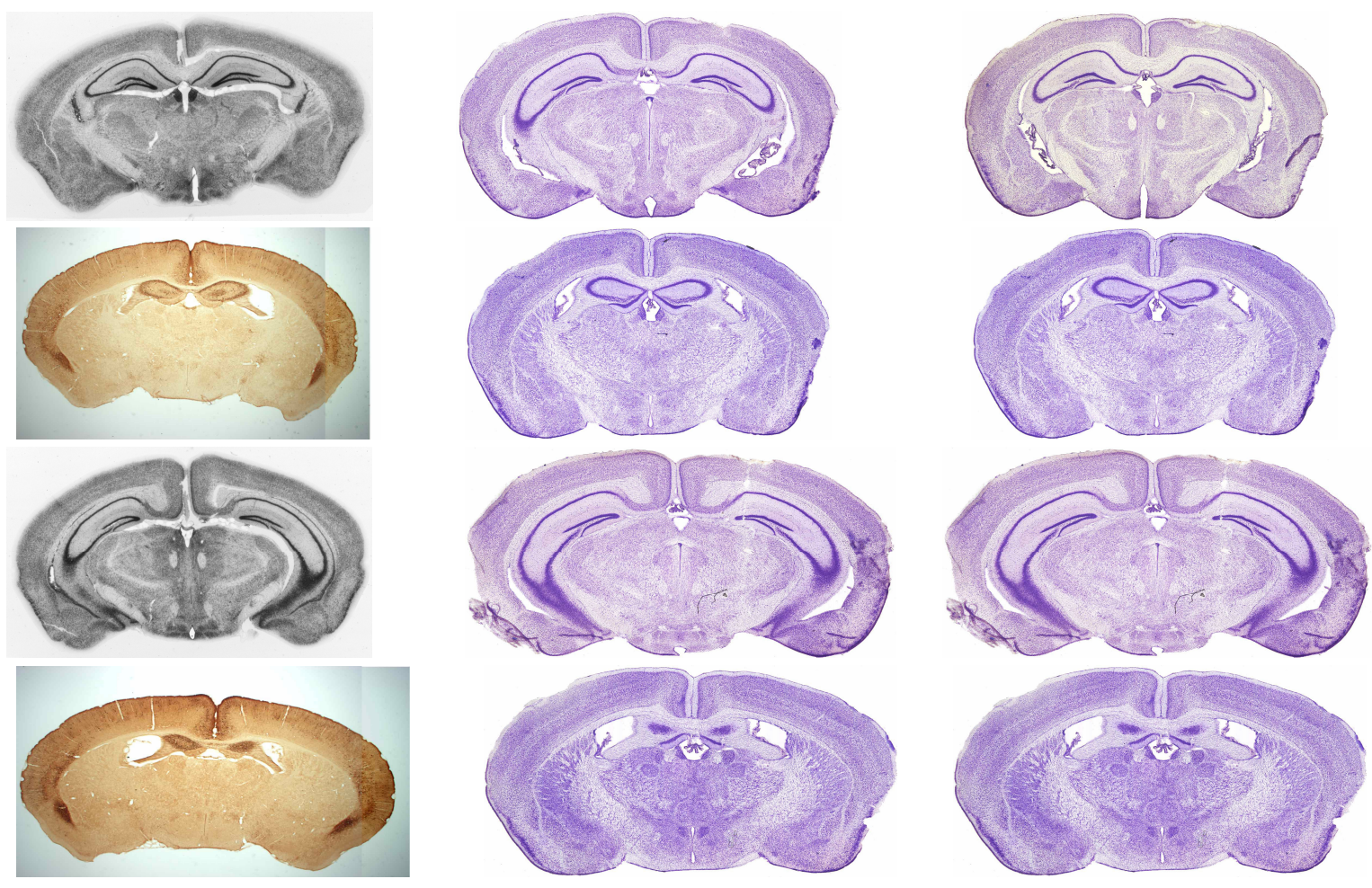

0

0

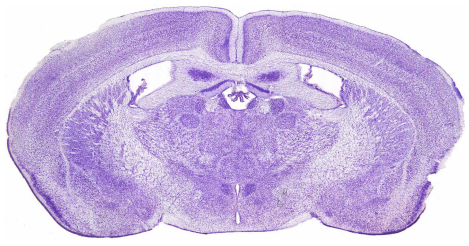

Figure 5.2 Examples of Query Results for Stage II 


\section{CONCLUSION AND FUTURE WORK}

\subsection{CONCLUSION}

One important task that is highlighted in our entire thesis is the mapping of the subject regions of interest with the corresponding reference regions of interest. Being an automated system, our goal is to achieve accurate mapping in the least possible time to submit mapped candidates to the next stage of decision-making. After identifying the corresponding plane of section through the atlas, experimental images are aligned with the atlas to study the internal structures of the brain image. We have developed an automatic image-atlas section-matching algorithm, which is a critical element in an atlasbased approach to macro-anatomical analysis of such sectional data. Mapping of brain histological images needs to process high resolution data. Hence, a mapping algorithm suitable for this application should have computational flexibility and scalability such that matching of large images is feasible.

In this thesis, a mapping algorithm is proposed that offers a trade-off between complexity and accuracy. A dual-stage shape matching technique is developed to classify the image and obtain an accurate match with the given query. The external contour of the brain section has been used for matching purpose, since this feature can be detected in most histological procedures. Image classification is studied using three methods: morphometrics based, Fourier based and radial distribution based approaches. It was observed that the best performance is achieved using radial distributions.

After the stage of classification, we focused on the hippocampus region for the finegrained mapping. We tested two techniques for image matching: Morphometrics-based and region-based template matching approaches. Though morphometrics features provided a high performance, they required user input. Thus, an automated technique was developed which involves texture based image matching. The developed technique can be applied to obtain best match for images in other groups by using a similar approach as described in this thesis. Further research is needed in order to perform alignment of the images with the template database. Also the effect of combining edge-features with texture and shape features can be studied. 


\subsection{FUTURE WORK}

For images derived from histological procedures, the need is for techniques to map the two-dimensional (2-D) images of the sectional material into the reference atlas which may be a full three-dimensional (3-D) data set or one consisting of a series of 2-D images. From the experimental results, it is clear that the developed technique gives better results for image mapping, but there are still a few areas to be explored. The image mapping can be done more efficiently using a segmentation technique. This issue can be addressed in the future to improve image mapping systems. Mapping to a 3-D data set can be explored. In this work, we have considered mapping coronal sections of the brain. Work needs to be done to study mapping and alignment of other sections such as sagittal brain sections. We believe that the work reported in this thesis can be extended to mapping using these other directions for slicing the mouse brain. 


\section{BIBLIOGRAPHY}

[1] Fernand S. Cohen, Zhengwei Yang, Zhaohui Huang, Jonathan Nissanov: "Automatic Matching of homologous sections", IEEE Transactions on Biomedical Engineering, vol. 45, no 5, pp. 642-649, May 1998

[2] L. Thurfjell, C. Bohm, and E. Bengtsson, "CBA: An atlas-based software tool used to facilitate the interpretation of neuroimaging data," Comput. Meth. Programs Biomed, vol. 47, pp. 51-71, 1995

[3] J. W. Haller, A. Banerjee, G. E. Christensen, M. Bado, S. Joshi, M.I. Miller, Y. Sheline, M. W. Vannier, and J. G. Csernansky, "Three dimensional hippocampal MR morphometry with high-dimensional transformation of a neuroanatomic atlas," Radiology, vol. 202, pp. 504-510, 1997

[4] Tao Ju, Joe Warren, Gregor Eichele, Christina Thaller, Wah Chiu and James Carson, "A geometry database for gene expression data", Proceedings of Eurographics Symposium on Geometry Processing, 2003

[5] J. Nissanov, S. Bhasin, G. Gregorious, D. McEachron, R. Paz, L. Rioux, and O. J. Tretiak, "Probability rat brain atlases: A tool for interpreting fused neuroanatomical maps," Soc. Neurosci. Abs., vol. 19, pp 545.5, 1993.

[6] G. Paxinos, C. Watson, M. Pennisi, and A. Topple, "Bregma, Lambda and the interaural midpoint in stereotaxic surgery with rats of different sex, strain and weight," $J$. Neurosci Methods, vol. 13, pp. 139-143, 1985.

[7] D. Wahlsten, W. J. Hudspeth, and K. Bernhardt, "Implications of genetic variation in mouse brain structure of electrode placement by stereotaxic surgery," J. Comput. Neurol., vol. 162, pp. 519-532, 1975.

[8] G. Hamarneh, J. Chen, B. Neiman, J. Henderson, M. Henkelman. "Segmentation, Registration, and Deformation Analysis of 3D MR Images of Mice”, Medical Image 
Computing and Computer-Assisted Intervention, 2003, Lecture Notes in Computer Science, ISBN 3-540-20464-4, vol. 2879, pp. 878-885

[9] Ioannis A. Kakadiaris, Musodiq Bello, Shiva Arunachalam, Wei Kang, Tao Ju, Joe Warren, James Carson, Wah Chiu, Christina Thaller, and Gregor Eichele, "Landmarkdriven, Atlas-based Segmentation of Mouse Brain Tissue Images Containing Gene Expression Data", Proceedings of MICCAI, pp.192-199, 2004

[10] J. P. Carson, T. Ju, C. Thaller, J. Warren, M. Bello, I. Kakadiaris, W. Chiu, G. Eichele, "Automated Characterization of Gene Expression Patterns with an Atlas of the Mouse Brain", Proceedings of Electrical Biomedical Engineering (EMBS), pp.29172920, 2004

[11] Flybrain, http://flybrain.neurobio.arizona.edu/Flybrain/html/

[12] Gary E. Christensen, Sarang C. Joshi, and Michael I. Miller, "Volumetric Transformation of Brain Anatomy", IEEE Transactions on Medical Imaging, vol. 16, no. 6, December 1997.

[13] Swanson, L. W., "Brain Maps: Structure of the Rat Brain", Elsevier, Amsterdam, Netherlands, 1992

[14] Paxinos, G., C. Watson," The Rat Brain in Stereotaxic Coordinates", Academic Press, San Diego, California 1998 November 2003, spiral bound, 120 pp, ISBN: 012547640X

[15] Toga, A. W., E. M. Santori, R. Hazani, K. Ambach. "Rat Atlas ImageDatabase", http://www.loni.ucla.edu/Research_Loni/atlases/rat/

[16] Franklin, B. J. Keith, G. Paxinos, "The Mouse Brain in Stereotaxic Coordinates". Academic Press, San Diego, California 2001.

[17] Toga, A. W. and Thompson, P. M., "New approaches in brain morphometry", American Journal of Geriatric Psychiatry, vol. 10, pp.13-23, 2002 
[18] Ron Kikinis, Martha E. Shenton, Dan V. losifescu, Robert W. McCarley, Pairash Saiviroonporn, Hiroto H. Hokama, Andre Robatino, David Metcalf, Cynthia G. Wible, Chiara M. Portas, Robert M. Donnino, and Ferenc A. Jolesz, "A digital brain atlas for surgical planning, mode1-driven segmentaion and teaching”, IEEE Transactions on Visualization and Computer Graphics, vol. 2, no. 3, September 1996

[19] Viergever, M. A., Maintz, J. B., and Stokking, R, "Integration of functional and anatomical brain images", Biophys. Chem., vol 68, no 1-3, 207-219, 1997, PMID: 9468620

[20] "Handbook of Immunohistochemistry and in situ Hybridization of Human Carcinomas", First Edition: Molecular Genetics, Gastrointestinal Carcinoma, and Ovarian Carcinoma, vol.4

[21] Young WS 3rd, Kuhar MJ, "A new method for receptor autoradiography: [3H] opioid receptors in rat brain", Brain Research, vol.179, no.2, pp.255-70, Dec28,1979 PubMed: 228806

[22] J.B.A. Mainz and M.A. Viergever, "A survey of medical image registration", Medical Image Analysis, vol 2, pp.1-36, 1998

[23] synapses.mcg.edu/ anatomy/rat/whole.stm

[24] MacKenzie-Graham A, Jones ES, Shattuck DW, Dinov ID, Bota M, Toga AW, "The informatics of a C57BL/6J mouse brain atlas", Neuroinformatics, vol 1, no 4, pp.393410, 2003, PMID: 15043223

[25] Allan MacKenzie-Graham, Erh-Fang Lee, Ivo D. Dinov, Mihail Bota, David W. Shattuck, Seth Ruffins, Heng Yuan, Fotios Konstantinidis, Alain Pitiot, Yi Ding, Guogang Hu, Russell E. Jacobs and Arthur W. Toga, "A multimodal, multidimensional atlas of the C57BL/ 6J mouse brain", Journal of Anatomy, vol 204, pp. 93-102, 2004.

[26] Wirtz, S. Fischer, B. Modersitzki, J. Schmitt, "Super-fast elastic registration of histologic images of a whole rat brain for three-dimensional reconstruction", International Society for Optical Engineering (SPIE), vol 5370; part 1, pp 328-334, 2004 
[27] www.mbl.org (Mouse Brain Library), University of Tennessee

[28] I.A. Bachelder, S. Ullman, "Contour Matching using Local Affine Transformations", Computer Vision and Pattern Recognition, 1992. Proceedings of CVPR '1992 IEEE Computer Sociey Conference, pp. 798-801, June 1992

[29] Dengsheng Zhang, Guojun Lu: Review of shape representation and description techniques. Pattern Recognition, vol.37, no.1, pp. 1-19, 2004

[30] Fernand S. Cohen, Zhengwei Yang, Zhaohui Huang, and Jonathan Nissanov, "Elastic 3-D Alignment of Rat Brain Histological Images", IEEE Transactions on Medical Imaging, vol. 22, no. 11, Nov 2003

[31] Z. Wang, Z. Chi and D. Feng, "Shape based leaf image retrieval", IEE Proceedings Vision, Image, and Signal Processing, vol 150, no 1, pp. 34-43, Feb 2003

[32] H. Kauppinen, T. Seppanen, M. Pietikainen, "An experimental comparison of autoregressive and Fourier-based descriptors in 2D Shape Classification", IEEE Trans. Pattern Analysis Machine Intell., vol. 17, no. 2, pp. 201-207, 1995.

[33] Stefan Muller, Stefan Eickeler, Gerhard Rigoll,, "Multimedia database retrieval using hand-drawn sketches", Fifth International Conference on Document Analysis and Recognition (ICDAR'99), pp. 289

[34] Greg Mori, Serge Belongie, and Jitendra Malik, "Efficient shape matching using shape contexts", IEEE Transactions on Pattern Analysis and Machine Intelligence, vol. 27 , no. 11,2005

[35] Dengsheng Zhang, Guojun Lu," A comparison of shape retrieval using Fourier descriptors and short-time Fourier descriptors", IEEE Pacific Rim Conference on Multimedia 2001, pp.855-860

[36] Cohen, Scott D, Guibas, Leonidas J, "Shape-based image retrieval using geometric hashing",DARPA97 , pp. 669-674, BibRef 9700 
[37] S. Antani, L.R. Long, G.R. Thoma, and D.J. Lee, "Anatomical shape representation in spine X-ray", [Proceeding] (396 )Visualization, Imaging, and Image Processing VIIP Sep 2003

[38] Sajjanhar, Lu, Zhang,"Image Retrieval Using Modified Generic Fourier Descriptors”

[39] Y. H. Ang, Z. Li, and S. H. Ong" Image retrieval based on multidimensional feature properties" Proceedings of IS\&T/SPIE Conference on Storage and Retrieval for Image and Video Databases III, 2420, pp.47- 57, 1995.

[40] [14] M. K Hu," Visual pattern recognition by moment invariants" IRE Transactions on Information Theory, vol.8,pp.179-187, 1962.

[41] [18] A. K. Jain and A. Vailaya" Shape-based retrieval: A case study with trademark image databases", Pattern Recognition, vol.31, no.9, pp.1369-1390, 1998.

[42] J. P. Eakins, J. D. Edwards, J. Riley, and P. L. Rosin," A comparison of the effectiveness of alternative feature sets in shape retrieval of multi-component images", In Proceedings of IS\&T/SPIE Conference on Storage and Retrieval for Media Databases 2001, Vol. SPIE 4315, pp.196-207, 2001.

[43] H. H. S. Ip, D. Shen, and K. K. T. Cheung,"Affine invariant retrieval of binary patterns using generalized complex moments,"In Second International Conference on Visual Information Systems (VISUAL'97), pages 301-308, 1997.

[44] E. M. Arkin, L. P. Chew, D. P. Huttenlocher, K. Kedem, and J. S. B. Mitchell,” An efficient computable metric for comparing polygon shapes", IEEE Transactions on Pattern Analysis and Machine Intelligence, vol.13,no.3,pp.209-216, 1991.

[45] L. J. Latecki and R. Lak"amper," Shape description and search for similar objects in image databases", In R. C. Veltkamp, H. Burkhardt, and H. P. Kriegel, editors, State-ofthe-Art in Content-Based Image and Video Retrieval, volume 22 of Computational Imaging and Vision, pp. 69-96. Kluwer Academic Publishers, 2001. 
[46] L. J. Latecki and R. Lak“amper," Application of planar shape comparison to object retrieval in image databases", Pattern Recognition, vol.35,no.1,pp.15-29, 2002.

[47] Angular Histograms for Shape Retrieval - Atul Sajjanhar Guojun

[48] A. Ferman, B. Giinsel, A. Tekalp, "Motion and Shape Signatures for Object-Based Indexing of MPEG-4 Compressed Video," icassp, p. 2601, 1997 IEEE International Conference on Acoustics, Speech, and Signal Processing (ICASSP'97), vol. 4, 1997

[49] D. Zhang, G. Lu'Evaluation of MPEG-7 shape descriptors against other shape Descriptors",Multimedia Systems 9, 2003, 1530.

[50] Jain AK, Duin RPW, Mao J., "Statistical pattern recognition: A review", IEEE Transactions on Pattern Analysis and Machine Intelligence, vol.22, no.1, pp.4-37, Jan 2000 .

[51]Dryden I, Mardia K,” Statistical Shape Analysis”, John Wiley \& Sons, Inc. 1998

[52] Statistical models of shape, http://www2.imm.dtu.dk/ aam/main/node12.html

[53] D. P. Huttenlocher, G. A. Klanderman, and W. J. Rucklidge," Comparing images using the Hausdorff distance", IEEE Trans. on Pattern Analysis and Machine Intelligence, vol.15, no.9, pp.850-863, 1993

[54] Loet Leydesdorff, "Similarity Measures, Author Cocitation Analysis, and Information Theory", Journal of the American Society for Information Science \& Technology JASIST 56(7), pp.769-772,2005

[55] Adrian N. Evans, "Cloud Tracking Using Ordinal Measures and Relaxation Labelling", University of Bath Department of Electronic and Electrical Engineering Claverton Down, Bath, BA2 7AY, United Kingdom

[56] C. Kuglin and D. Hines," The Phase Correlation Image alignment Method”, IEEE Int. Conf. on Cybernetics and Society, 1975 
[57] Koprinska, I. \& Carrato, S. (2001), "Temporal video segmentation: A survey", Signal Processing: Image Communication, vol.16,no.5,pp. 477-500

[58] D.I. Barnea and H.F. Silverman, "A Class of Algorithms for Fast Digital Registration”, IEEE Transactions on Computers, C-21, pp.179-186,1972

[59] H.G.Barrow, J.M. Tenenbaum, R.C.Bolles and H.C.Wolf, "Parametric Correspondence and Chamfer Matching: Two new techniques for image matching", Proceedings of International Joint Conference in Artificial Intelligence, pp.659-663, 1977

[60] J. Canny," A Computational Approach to Edge Detection", IEEE Transactions on Pattern Analysis and Machine Intelligence, Vol 8, No. 6, Nov 1986

[61] B.S. Majunath and W.Y. Ma, "Texture features for browsing and retrieval of image data," IEEE Trans. Pattern Analysis and Machine Intelligence, vol.18, no.8, pp.837- 842, August.1996

[62] H. Lilian Tang, Rudolf Hanka, "Histological image retrieval based on semantic content analysis", IEEE Transactions on Information Technology in Biomedicine, vol. 7, no. 1 , pp. 26-36, 2003

[63] Biomedical Neuroinformatics Research Network (BIRN) http://www.nbirn.net/

[64] Neuroimaging Analysis center http://spl.harvard.edu:8000/pages/projects/grants/nac/

[65] Yunyong Ma, Hang Hu, Albert S. Berrebi, Peter H. Mathers, and Ariel Agmon Distinct Subtypes of Somatostatin-Containing Neocortical Interneurons Revealed in Transgenic Mice J. Neurosci. 2006 26: 5069-5082 


\section{APPENDIX}

\section{PRE-DEFINED IMAGE CLASSIFICATION}

\section{CLASS I}

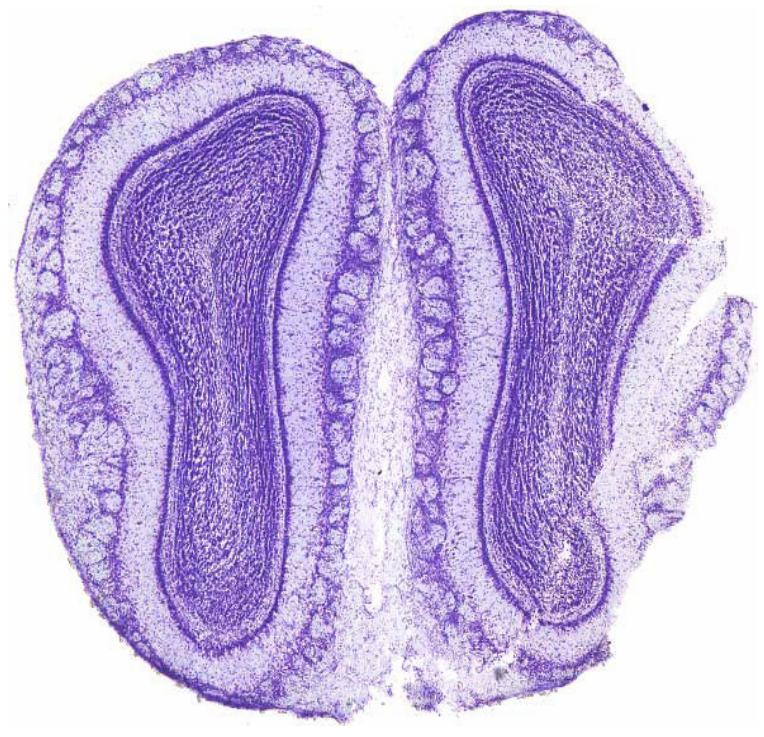

Image1

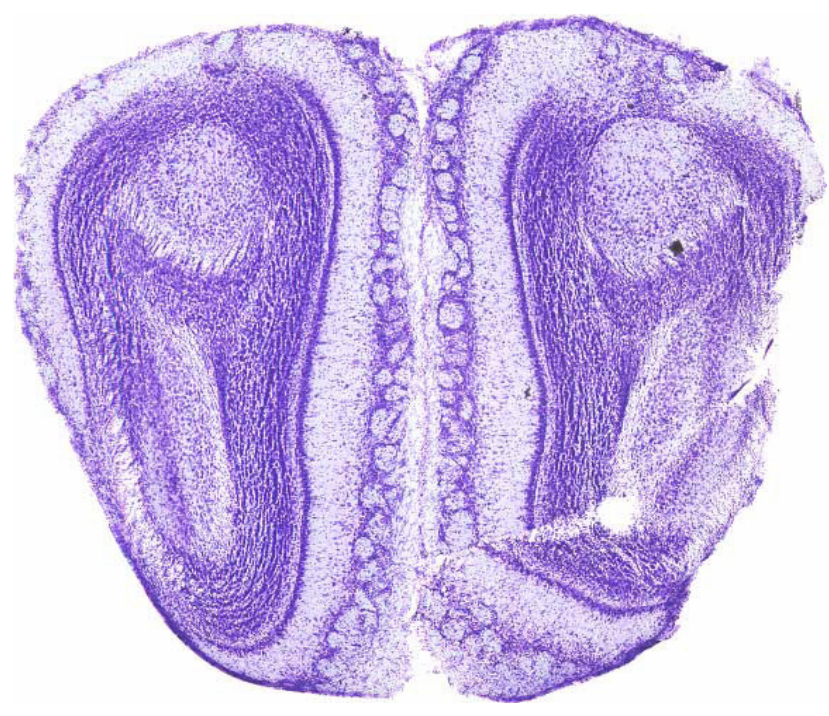

Image 2 


\section{CLASS II}

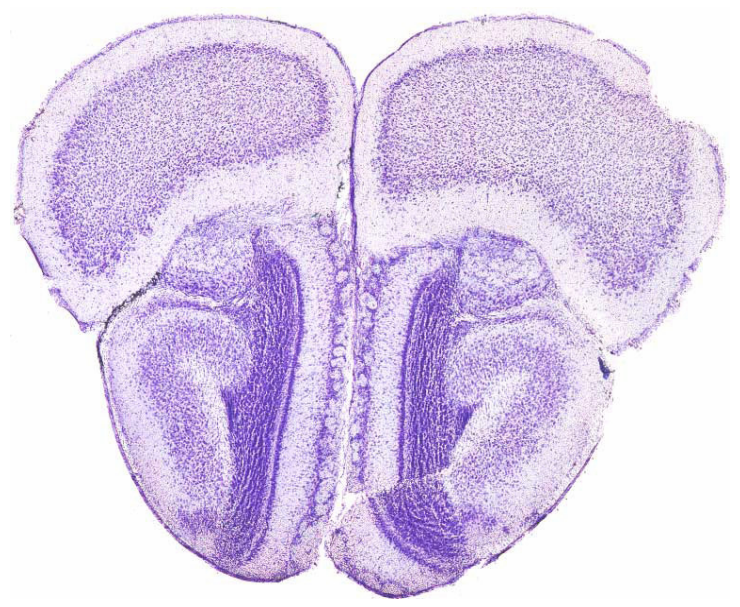

Image 3

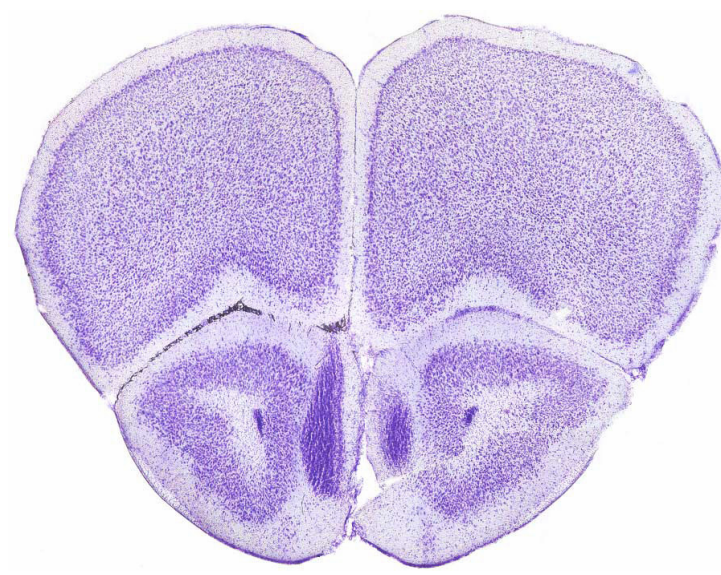

Image 5

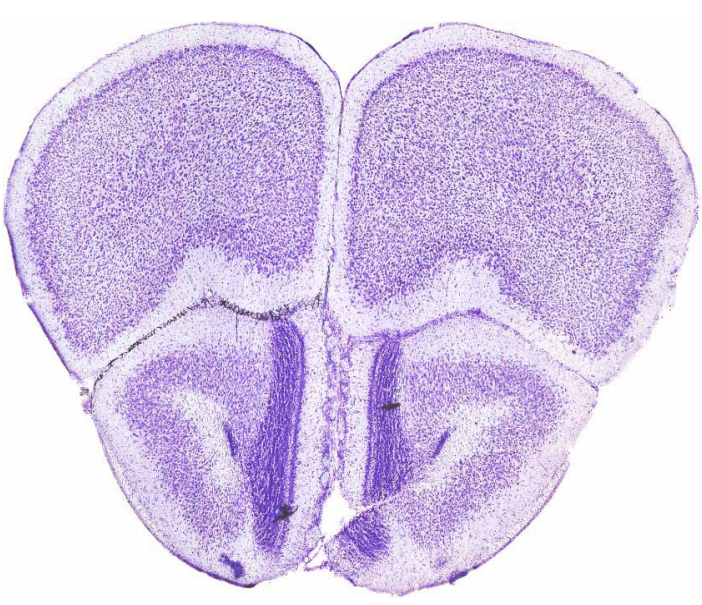

Image 4

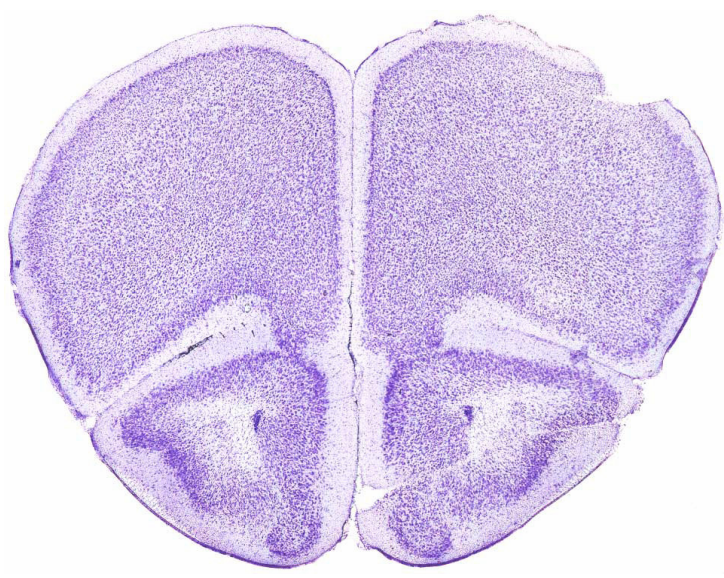

Image 6

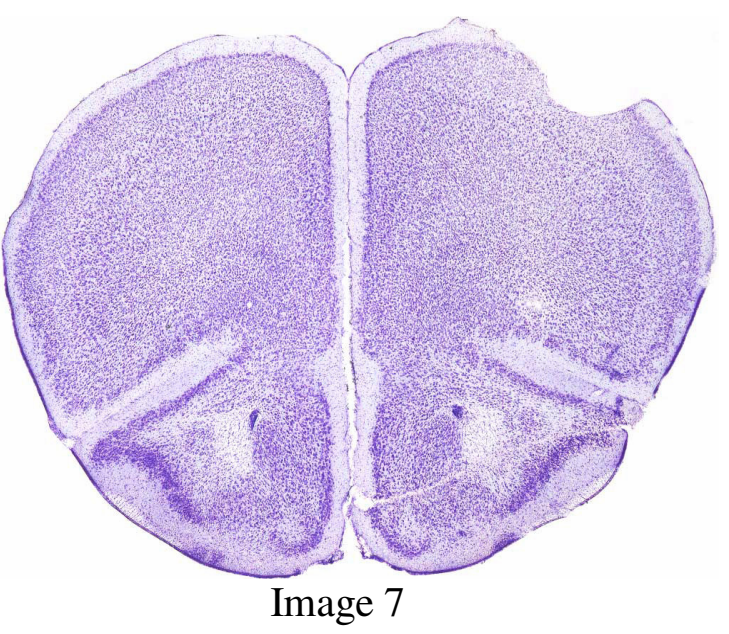




\section{CLASS III}

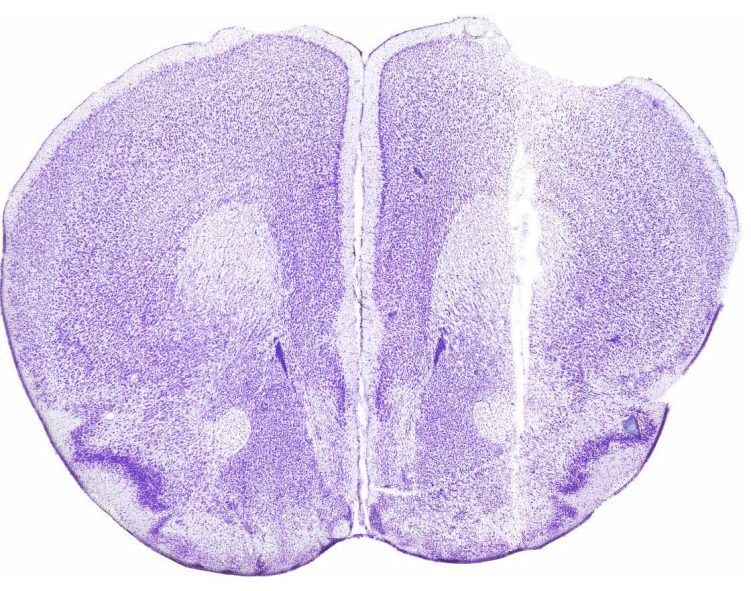

Image 8

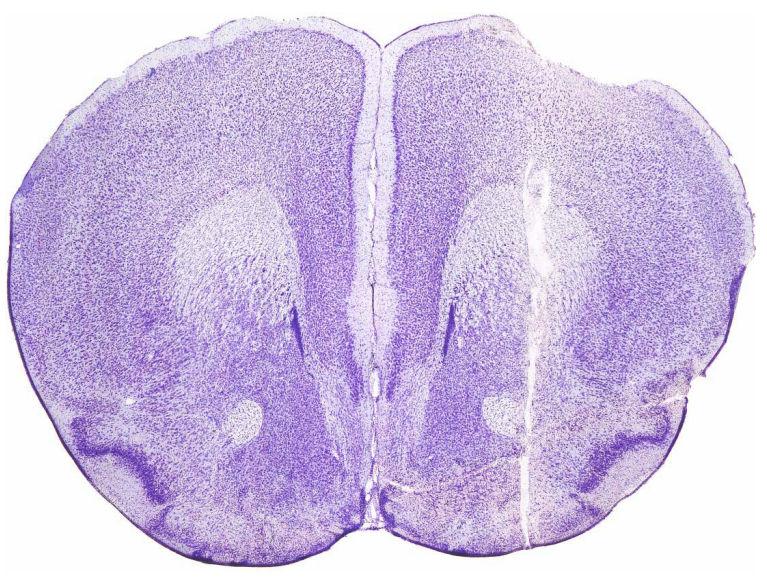

Image 9

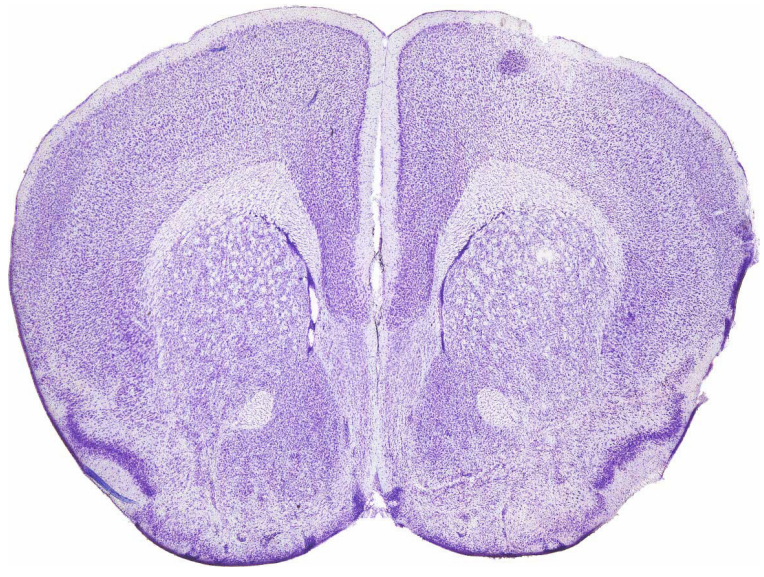

Image 10 


\section{CLASS IV}

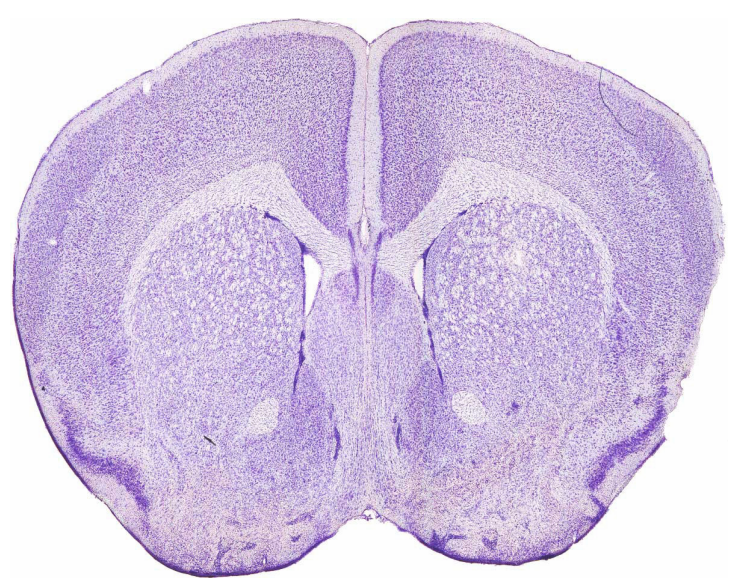

Image 11

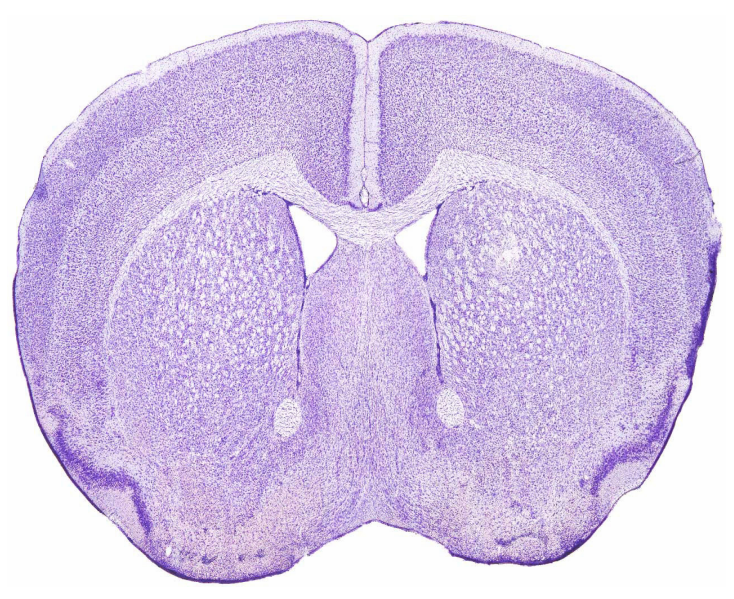

Image 13

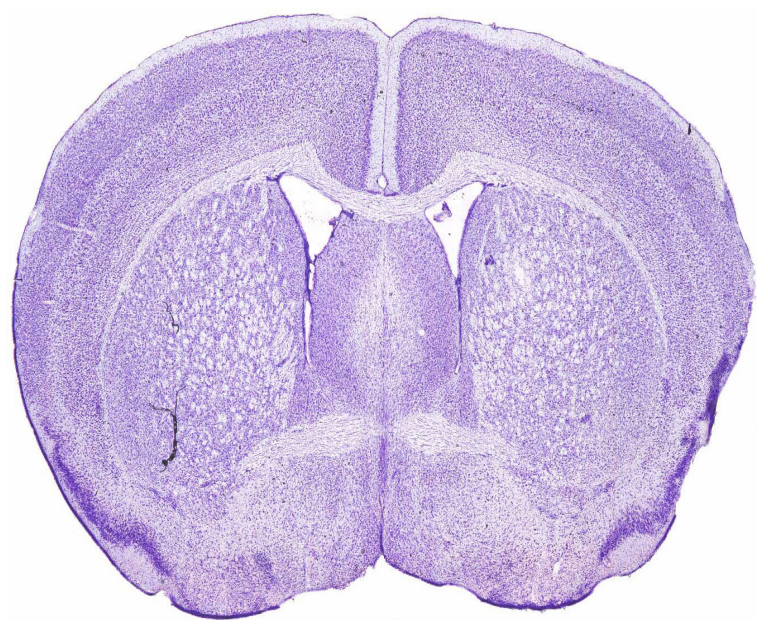

Image 15

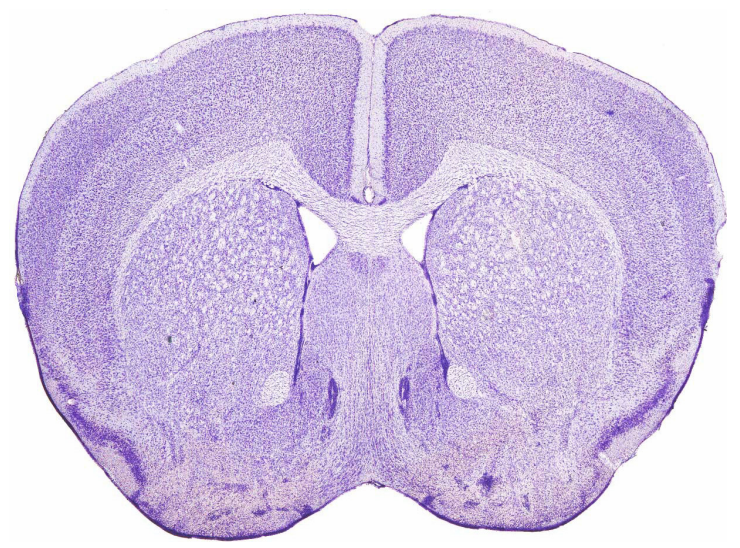

Image 12

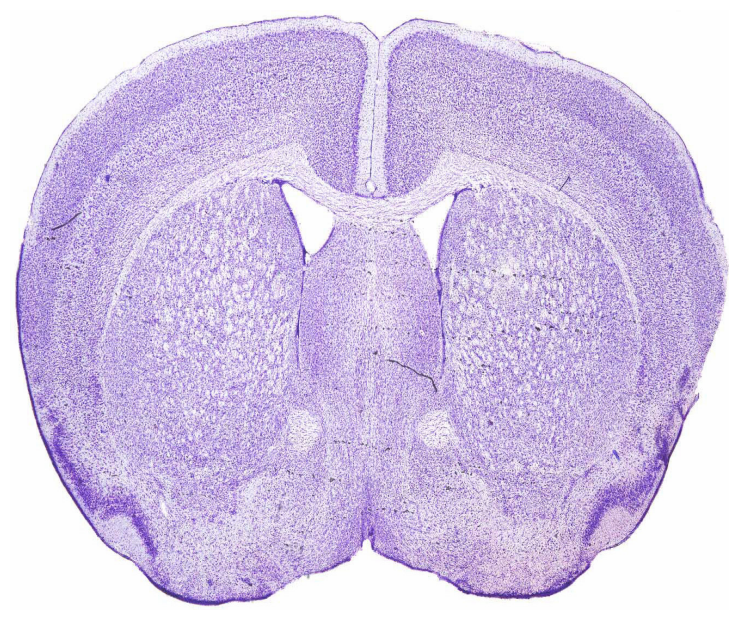

Image 14 


\section{CLASS V}

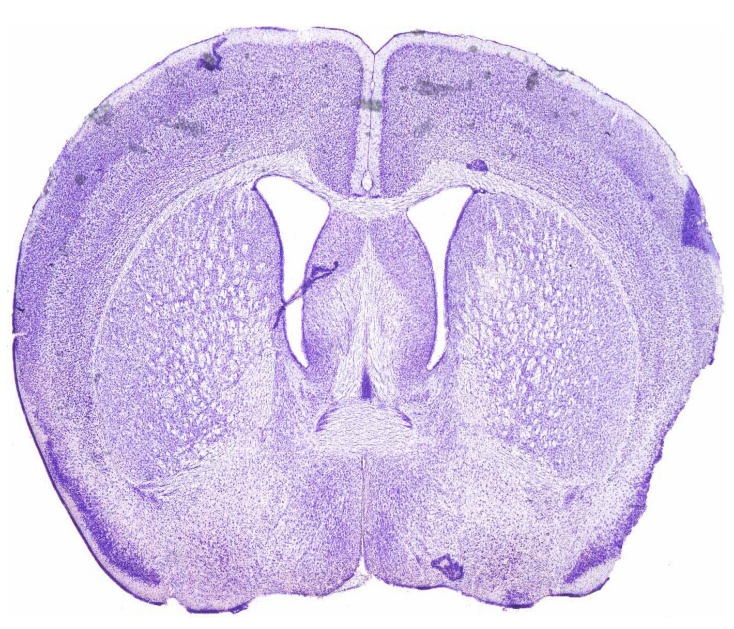

Image 16

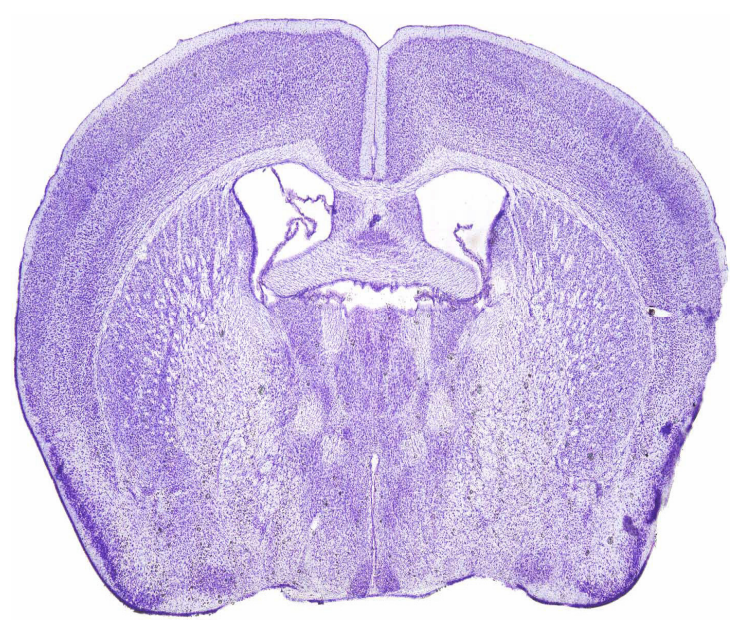

Image 18
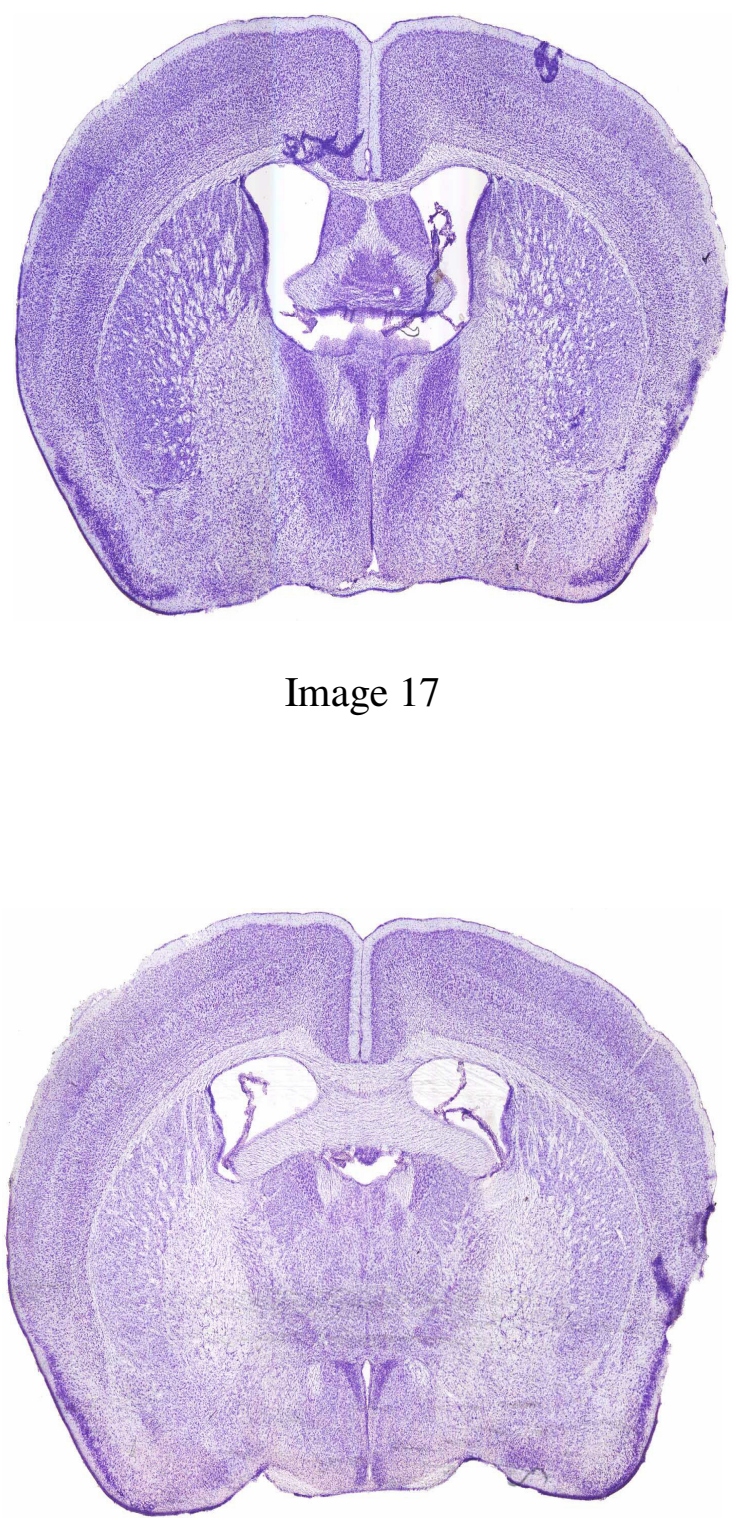

Image 19 


\section{CLASS VI}
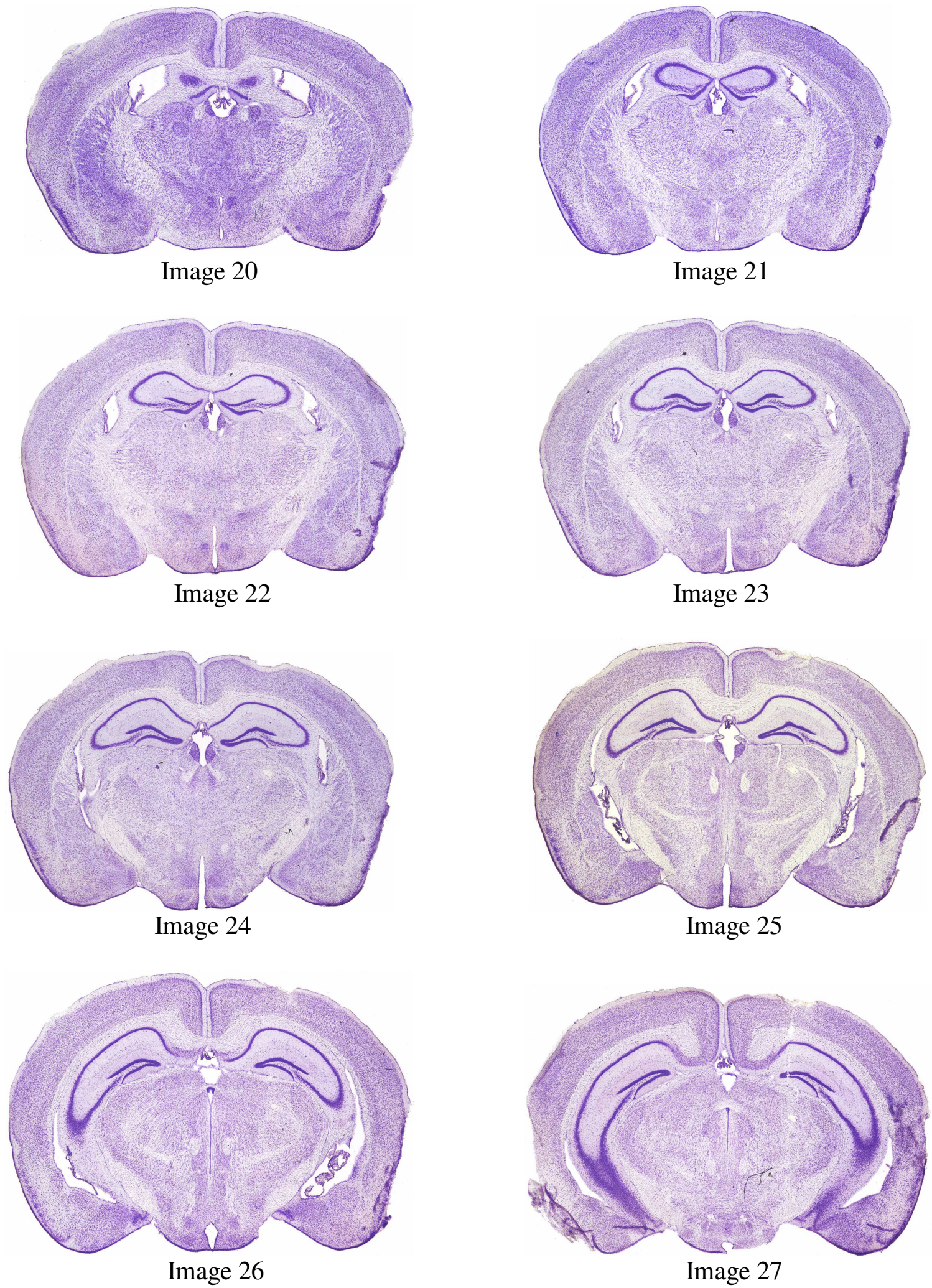


\section{CLASS VII}

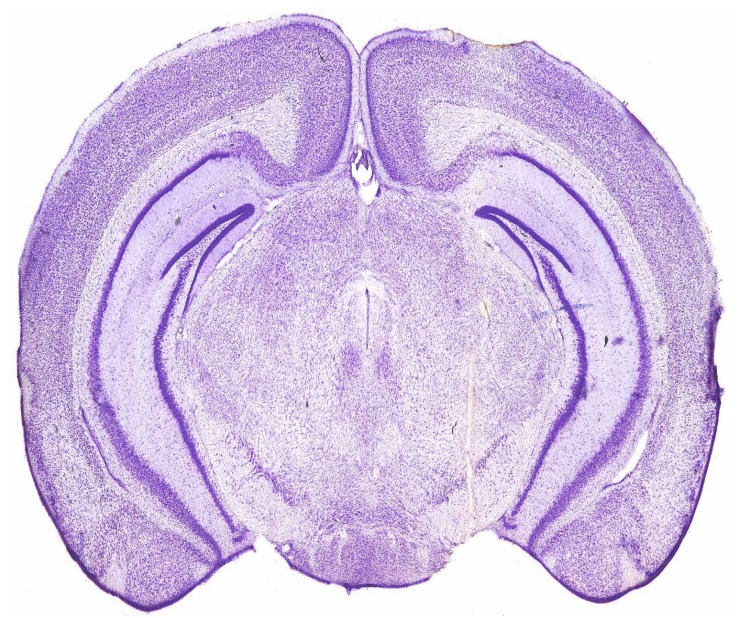

Image 28

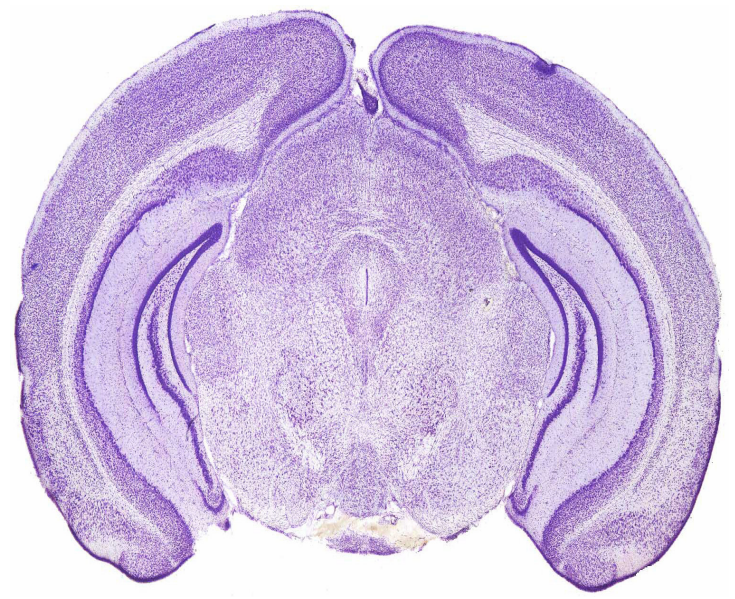

Image 30

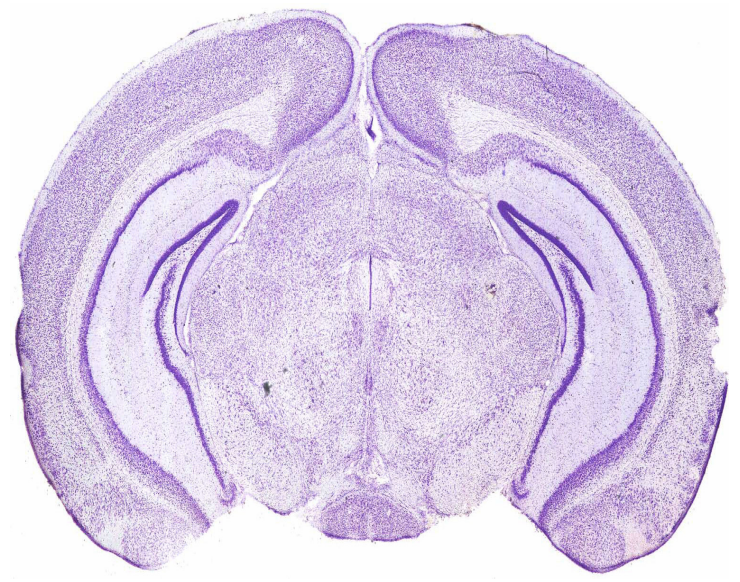

Image 29

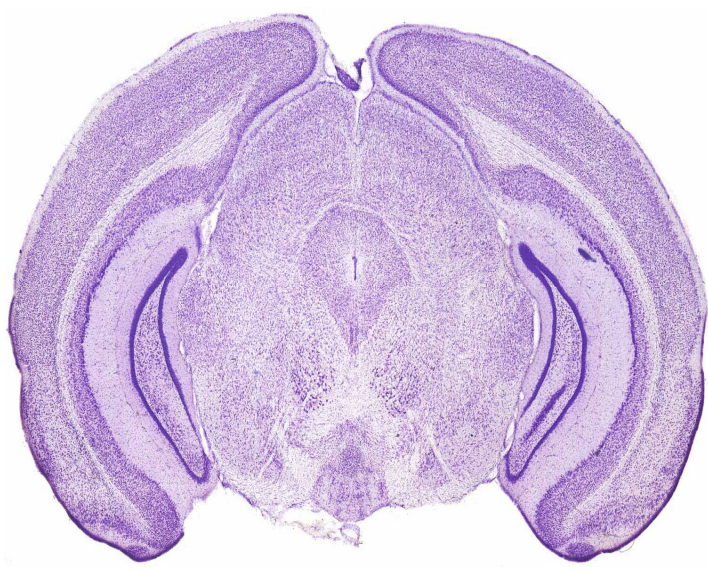

Image 31

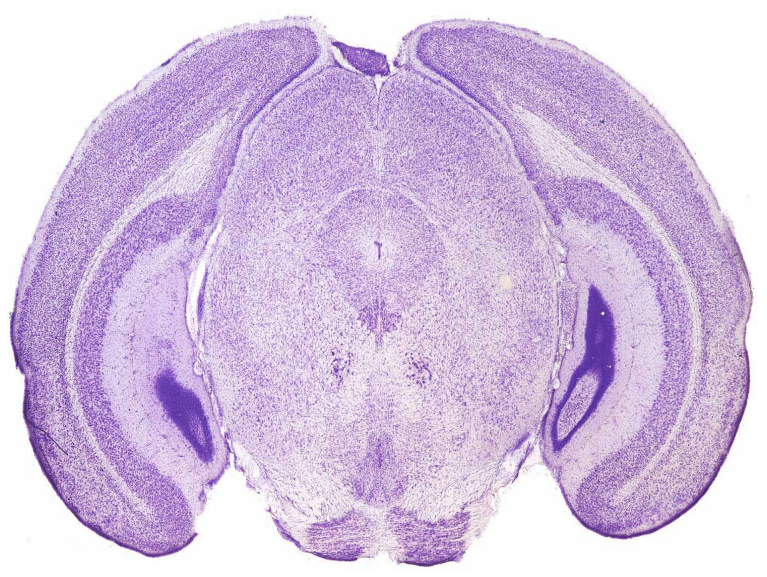

Image 32 


\section{CLASS VIII}

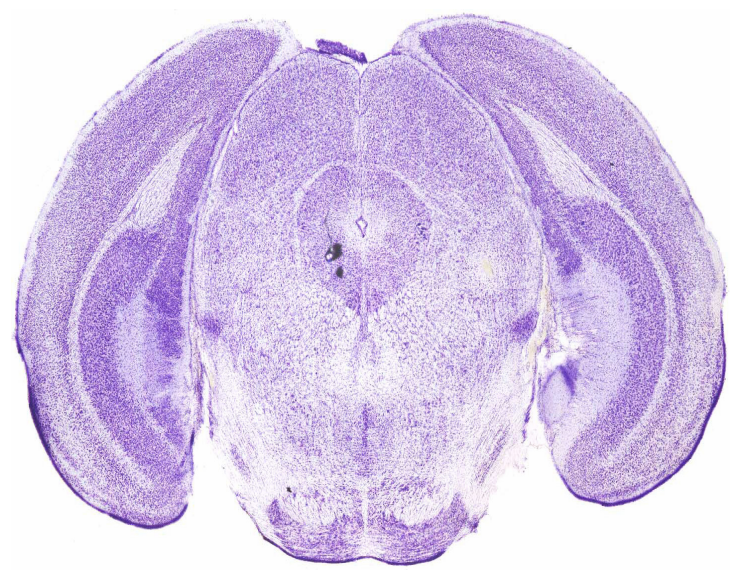

Image 33

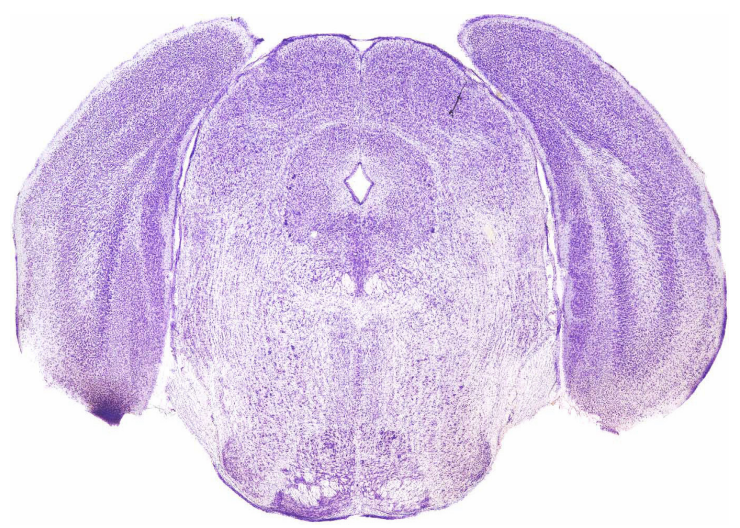

Image 35

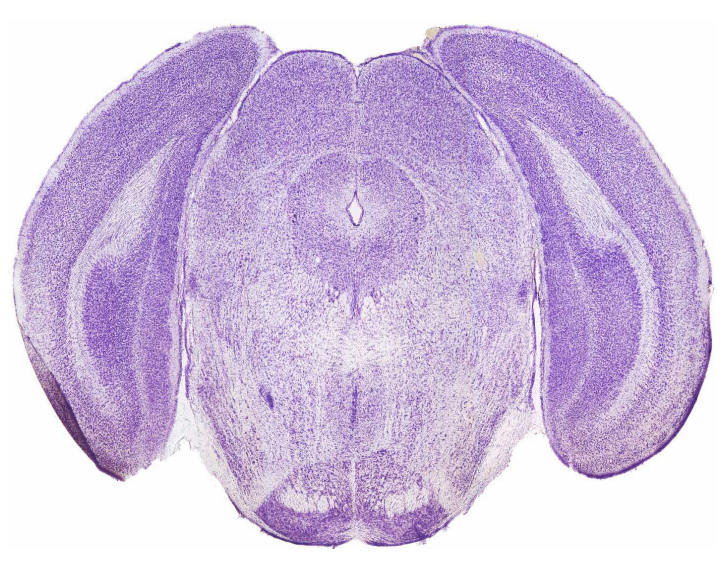

Image 34

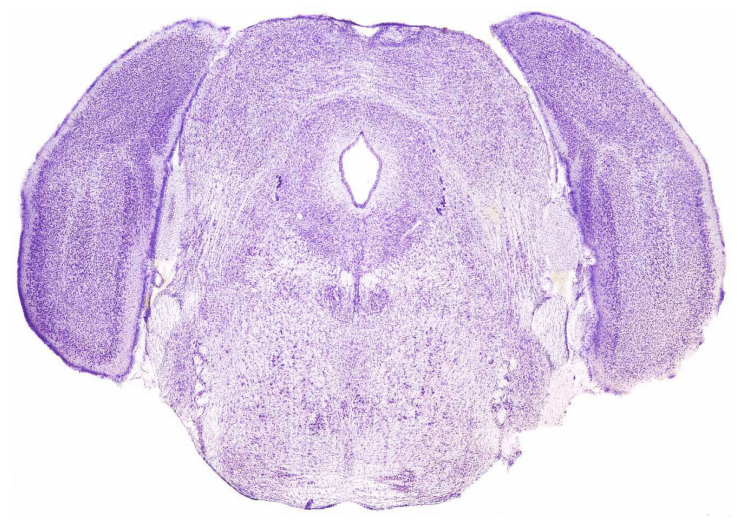

Image 36

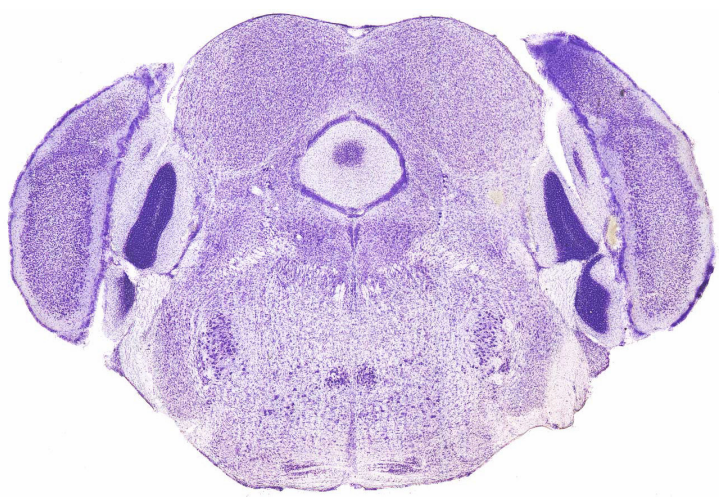

Image 37 


\section{CLASS IX}
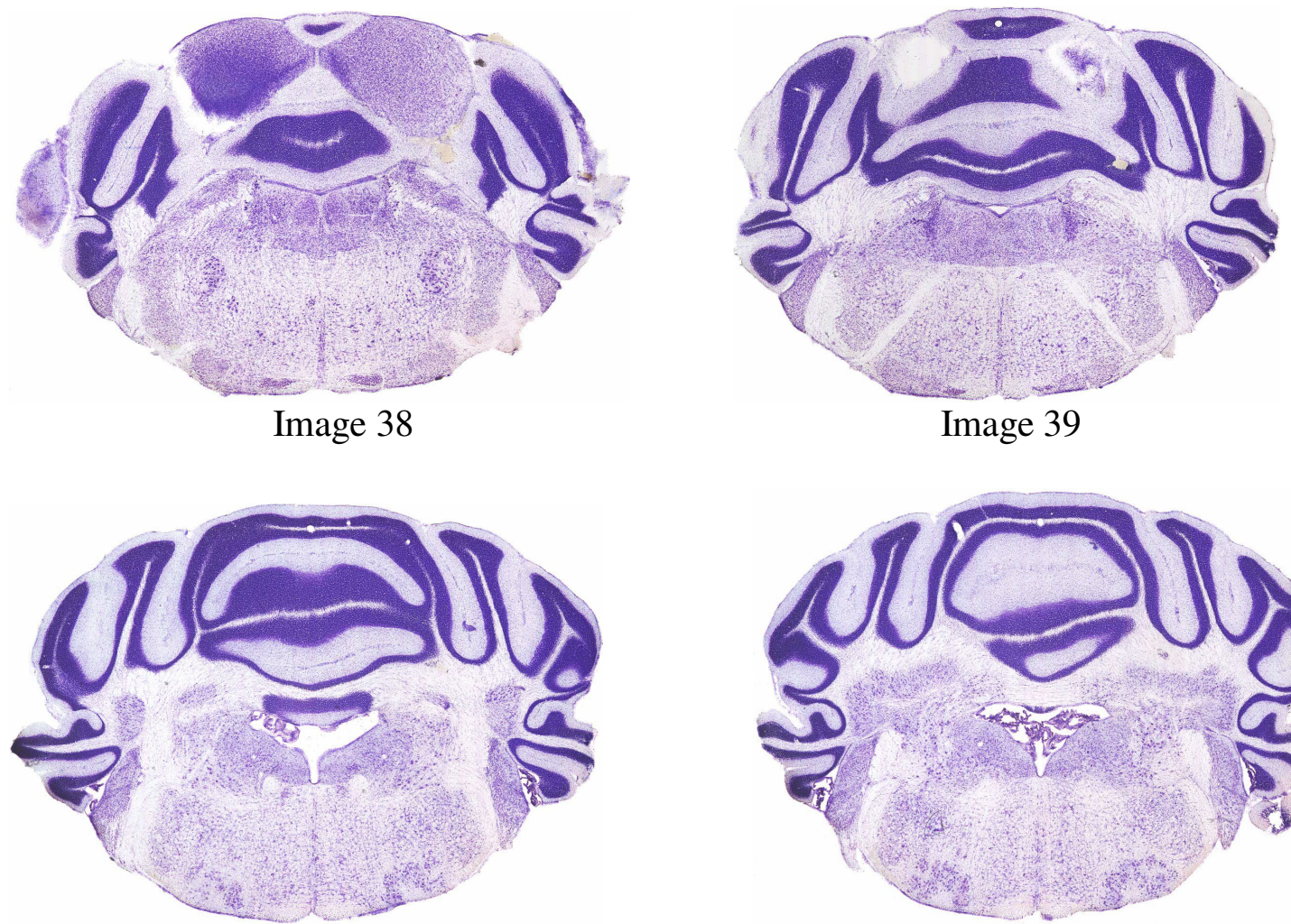

Image 40

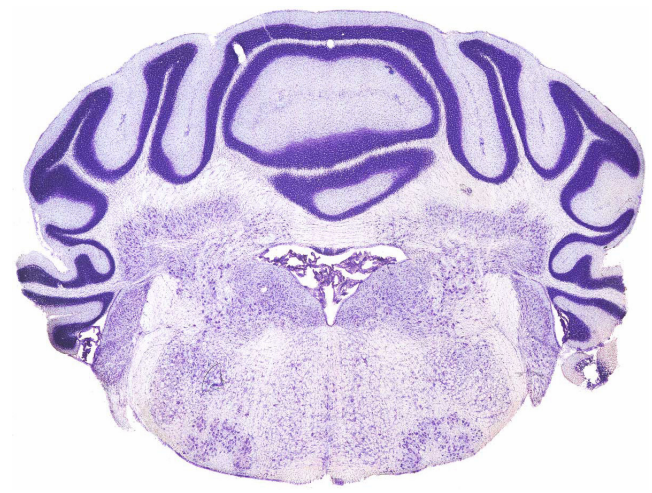

Image 41
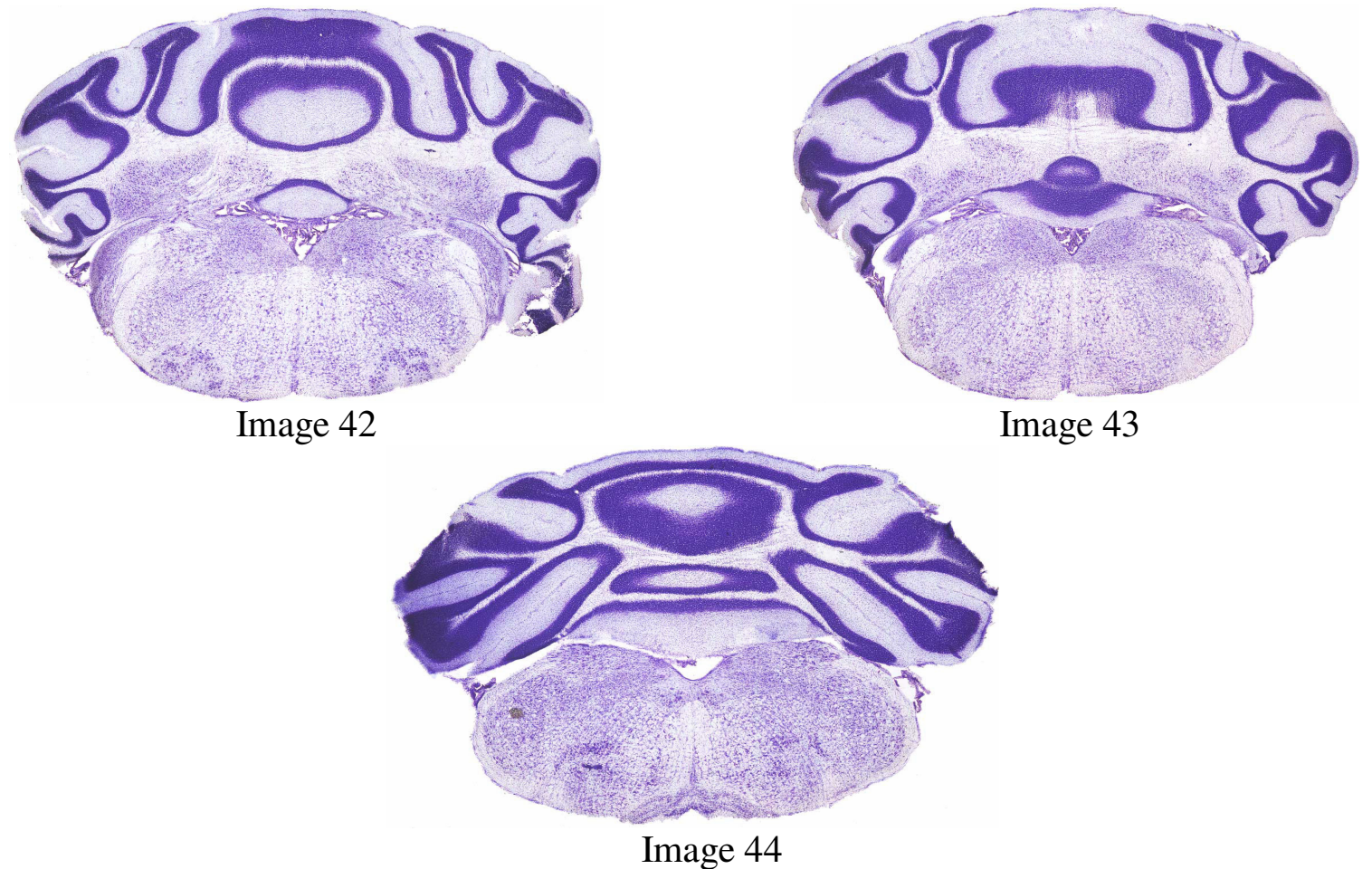


\section{CLASS X}
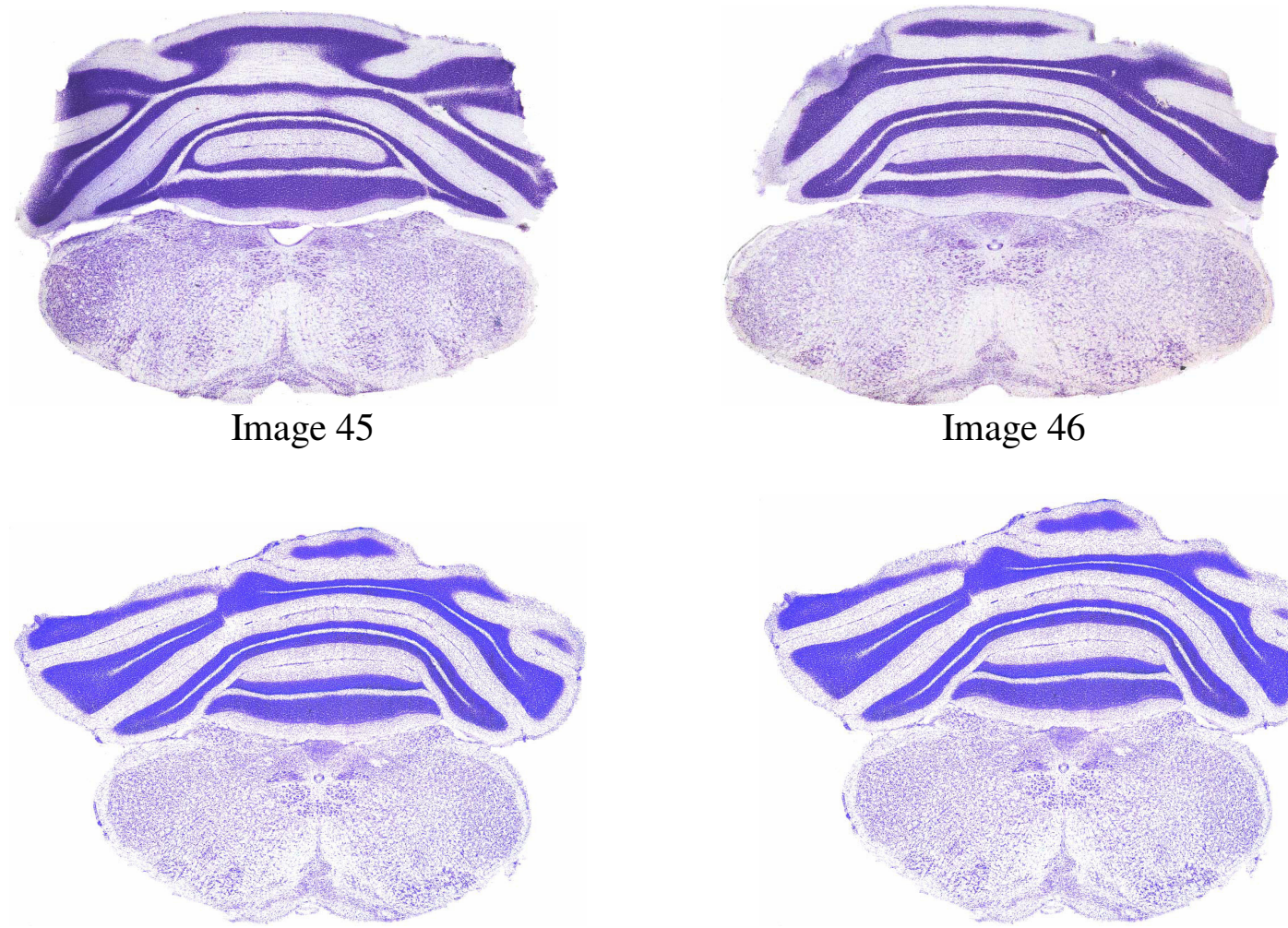

Image 47
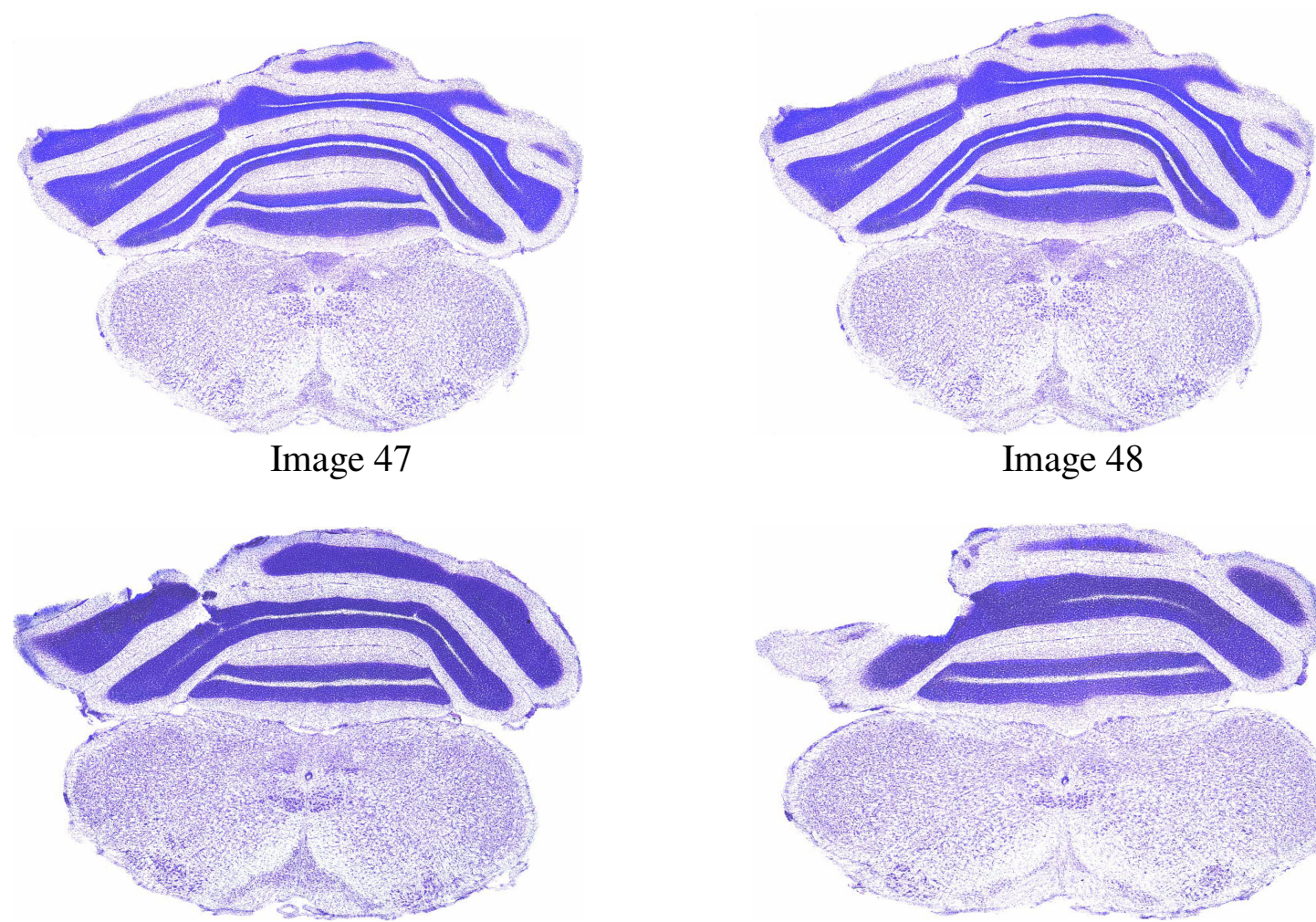

Image 49

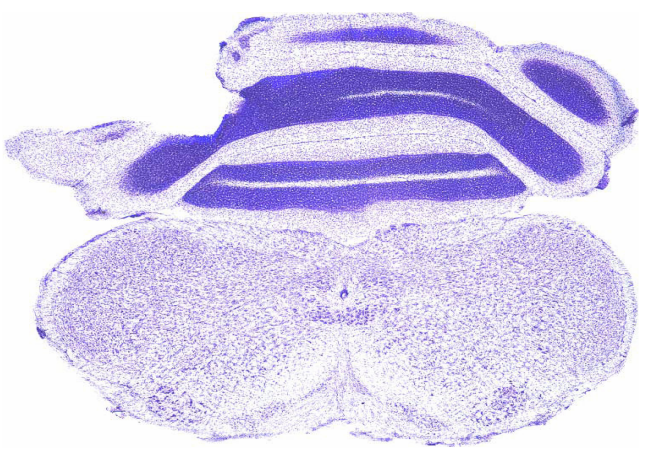

Image 50

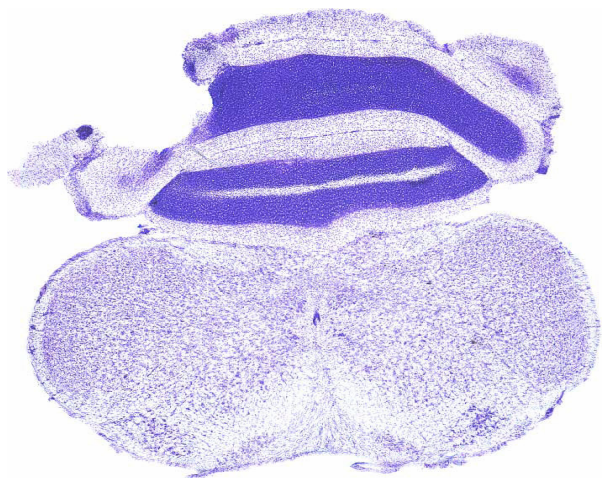

Image 51 
CLASS XI

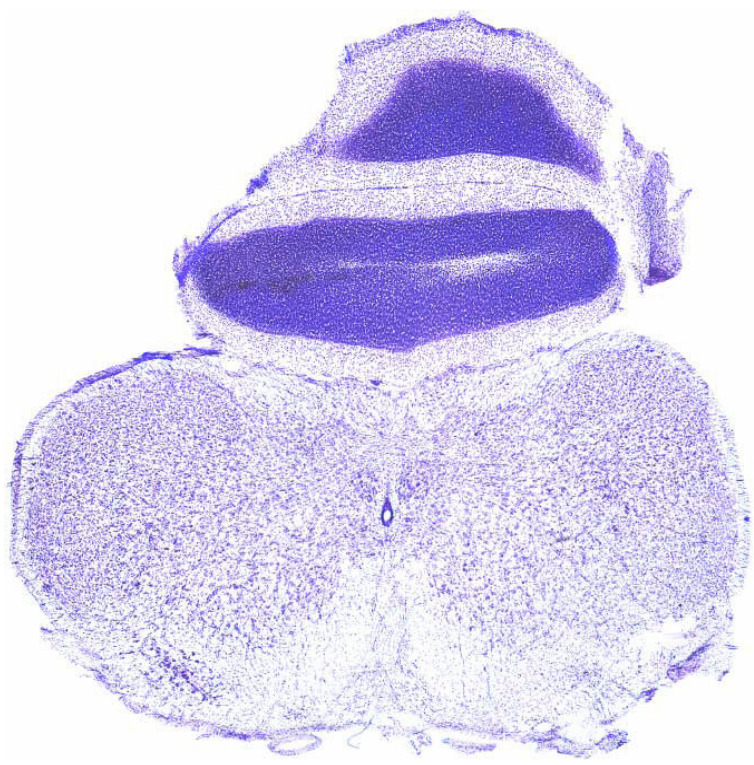

Image 52

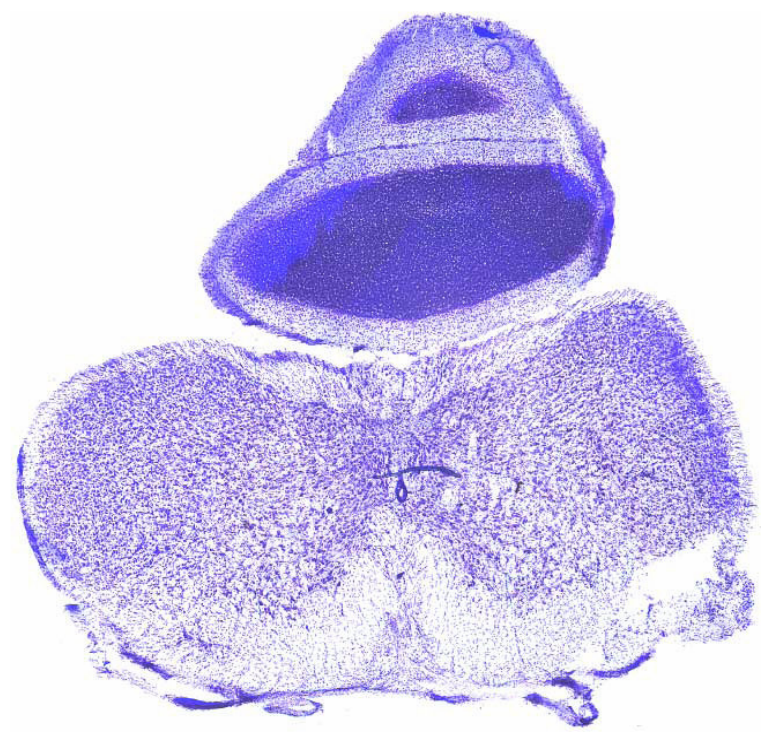

Image 53

68 